\title{
Novel Strategies to design Controllers and State-Predictors based on Disturbance Observers
}

September, 2020

Author: Alberto Castillo

Supervisors: Pedro García Gil

Pedro Albertos Perez 

One of the main concerns of the control theory is how to develop algorithms that regulate, learn and predict how the systems behave. Today, these algorithms are being run in computers that automatically control hundreds of processes that directly affect to our daily lives; but, unfortunately, just few people know about them and how and why they work.

Thanks to all that have contributed -or are contributing- to develop and understand this interesting field of research.

A. Castillo 



\section{Preface}

Engineering or physical systems are used to be uncertain. Its uncertainty is manifested whenever the system shows behaviors that are relatively different than the ones predicted by its model; being mostly caused by: modeling errors; unknown dynamics; changes in the system properties; random interactions with other systems; or changes in the operating conditions.

Through the last 40 years, it has been persistently proved that the system uncertainties could have very negative effects in the performance of a feedback regulator if they are not properly considered in the mathematical formulations of the employed algorithms. Thus, an important part of the recent research is focused on this topic; searching for the most appropriate ways to mathematically represent the system uncertainties and looking for new mathematicaltools that permit to make use of such uncertainty-representation in order to design robust control algorithms.

In this thesis, new contributions in this line are provided. Concretely, novel methodologies to design Disturbance Observer-Based Controllers (DOBCs) and Predictors (DOBPs) for uncertain dynamic systems are developed. The main contribution is to show that the DOBCs can be constructed from an optimality-based approach, with the main objective of approximating the -unrealizable- optimal control signal that minimizes a quadratic-cost performance index subject to a LTI disturbed model constraint. This novel robust control design is indistinctly valid for SISO/MIMO models with single/multiple matched/mismatched disturbances; offering also a highly intuitive and versatile 
tuning through the weighting matrices. Similarly, the DOBPs are synthesized in order to approximate the time-domain solution of LTI disturbed models.

For the sake of completeness, the document also includes a brief review of the main robust control methods and the impact that they have had on the technological revolution of the 21st century; some discussions about the usefulness of the LTI disturbed models for representing uncertain dynamic systems; and different relationships, comparisons and numerical simulations, of the proposed methods with other control approaches. 


\section{Prefacio}

Los sistemas de ingeniería o físicos suelen ser inciertos. Su incertidumbre se manifiesta cuando el sistema muestra comportamientos que son relativamente diferentes a los que su modelo predice; estando principalmente causada por: errores de modelado; dinámicas desconocidas; cambios en las propiedades del sistema; interacciones aleatorias con otros sistemas; o cambios en las condiciones de operación.

Durante los últimos 40 años, se ha demostrado reiteradamente que las incertidumbres de los sistemas pueden tener efectos muy negativos sobre el comportamiento de un controlador si éstas no se consideran adecuadamente sus formulaciones matemáticas. Por esta razón, una parte importante de la investigación actual está centrada en este tema; buscando las formas mas adecuadas para representar matemáticamente las incertidumbres de los sistemas, así como buscando nuevas herramientas matemáticas que permitan hacer uso de ésta representación de la incertidumbre con el objetivo de diseñar algoritmos de control robustos.

En esta tesis se presentan nuevas aportaciones en esta línea. Concretamente, se desarrollan nuevas metodologías para diseñar controladores (DOBCs) y predictores (DOBPs) para sistemas dinámicos inciertos basados en observadores de perturbaciones. La principal aportación es demostrar que los DOBCs se pueden sintetizar desde un enfoque de control óptimo; siendo su principal criterio de diseño el de aproximar la -irrealizable- señal de control óptima que minimiza un índice de coste cuadrático sujeto a un modelo dinámico lineal (LTI). Este nuevo enfoque de diseño es indistintamente válido para modelos 
SISO/MIMO con múltiples o únicas perturbaciones. Además permite un ajuste del controlador muy intuitivo gracias a las matrices de ponderación del coste. De forma similar; los DOBPs se construyen con el objetivo de aproximar la solución temporal un sistema dinámico perturbado.

Con el objetivo de contextualizar la aportación, el documento también incluye un breve resumen de los principales métodos de control robusto y el impacto que han tenido en la revolución tecnológica del siglo XXI; algunas discusiones sobre la utilidad de los modelos LTI perturbados para representar sistemas dinámicos inciertos; y algunas relaciones, comparaciones y simulaciones numéricas de los métodos propuestos con otras técnicas de control. 


\section{Prefaci}

Els sistemes d'enginyeria o físics solen ser incerts. La seua incertesa es manifesta quan el sistema mostra comportaments que són relativament diferents als que el seu model prediu; sent principalment causada per: errors de modelatge; dinàmiques desconegudes; canvis en les propietats del sistema; interaccions aleatòries amb altres sistemes; o canvis en les condicions d'operació.

Durant els últims 40 anys, s'ha demostrat reiteradament que les incerteses dels sistemes poden tindre efectes molt negatius sobre el comportament d'un controlador si aquestes no es consideren adequadament les seues formulacions matemàtiques. Per aquesta raó, una part important de la investigació actual està centrada en aquest tema; buscant les formes mes adequades per a representar matemàticament les incerteses dels sistemes, així com buscant noves tècniques matemàtiques que permeten fer ús d'aquesta representació de la incertesa amb l'objectiu de dissenyar algorismes de control robustos.

En aquesta tesi es presenten noves aportacions en aquesta línia. Concretament, es desenvolupen noves metodologies per a dissenyar controladors (DOBCs) i predictors (DOBPs) per a sistemes dinàmics incerts basats en observadors de pertorbacions. La principal aportació és demostrar que els DOBCs es poden sintetitzar des d'un punt de vista de control òptim; sent el seu principal criteri de disseny el d'aproximar la -irrealitzable- senyal de control òptima que minimitza un índex de cost quadràtic restringit a un model dinàmic lineal (LTI). Aquest nou plantejament és indistintament vàlid per a models SISO/MIMO amb múltiples o úniques pertorbacions. A més permet un ajust del controlador molt intuitiu gràcies a les matrius de ponderació del cost. De manera similar; 
els DOBPs es construeixen amb l'objectiu d'aproximar la solució temporal un sistema dinàmic pertorbat.

Amb l'objectiu de contextualitzar l'aportació, el document també inclou un breu resum dels principals mètodes de control robust i l'impacte que han tingut en la revolució tecnològica del segle XXI; algunes discussions sobre la utilitat dels models LTI pertorbats per a representar sistemes dinàmics incerts; i algunes relacions, comparacions i simulacions numèriques dels mètodes proposats amb altres tècniques de control. 


\section{Contents}

Preface $\quad$ V

Index $\quad$ xi

Glossary $\quad$ xi

1 Introduction 1

1.1 Introduction: robust control of uncertain systems $\ldots \ldots \ldots \ldots \ldots \ldots$

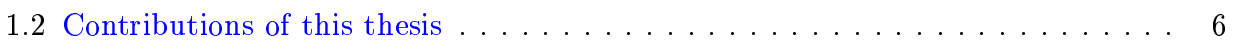

1.3 Background: why this thesis was developed and its main motivations . . . . 8

1.4 Chapters organization. . . . . . . . . . . . . . . 11

2 Problem statement 13

2.1 LTI disturbed models: a simple framework to deal with uncertain systems. . . 14

2.2 DOBs: numerical methods for estimating disturbance signals . . . . . . . . 18

2.3 Definition of the optimal controller, $u_{\gamma}$, and the state-predictor, $x\left(t+\Delta_{t}\right) \ldots 19$

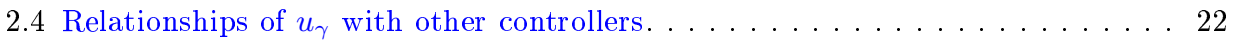

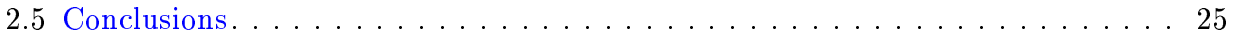


3 DOBC: Optimality-based design, $u_{\gamma}(t) \quad 27$

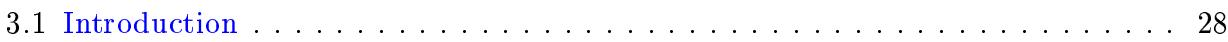

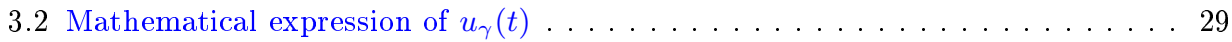

3.3 Synthesizing DOBCs that approximately implement $u_{\gamma}(t) \ldots \ldots \ldots \ldots 32$

3.4 Application: design of a high-order ESO-based controller . . . . . . . . . . . . 34

3.5 Comparative example: magnetic levitation control. . . . . . . . . . . 37

3.6 Comparative example: closed-loop glucose control . . . . . . . . . . . . . 42

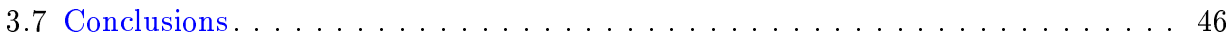

4 DOBC: Stability conditions 49

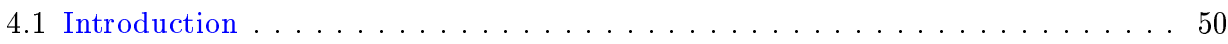

4.2 Closed-loop stability conditions $\ldots \ldots \ldots \ldots \ldots \ldots \ldots \ldots$

4.3 Numerical optimization of the stability conditions . . . . . . . . . . 57

4.4 An illustrative simulation $\ldots \ldots \ldots \ldots \ldots \ldots \ldots \ldots$

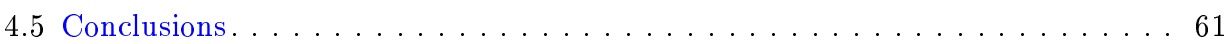

5 DOBP: Design based on the model's solution, $x\left(t+\Delta_{t}\right)$. 63

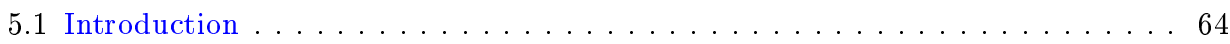

5.2 Synthesizing DOBPs that approximate $x\left(t+\Delta_{t}\right) \ldots \ldots \ldots \ldots \ldots$

5.3 Application: design of a high-order ESO-based predictor . . . . . . . . . 69

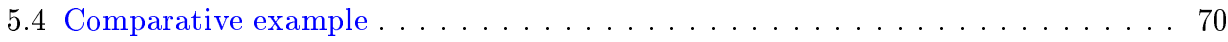

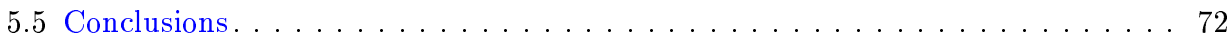

$\begin{array}{ll}\text { Conclusions and future works } & 73\end{array}$

$\begin{array}{ll}\text { Bibliography } & 77\end{array}$

Annex 1. Proof of Theorem 4.2.1 91

Annex 2. MatLab ${ }^{\circledR}$ implementations $\quad 95$ 


\section{Glossary}

$\begin{array}{ll}\text { ADRC } & \text { Active Disturbance Rejection-based Control } \\ \text { ARE } & \text { Algebraic Ricatti Equation } \\ \text { DOB } & \text { Disturbance Observer } \\ \text { DOBC } & \text { Disturbance Observer-based Control } \\ \text { DOBFD } & \text { Disturbance Observer-based Failure Detection } \\ \text { DOBP } & \text { Disturbance Observer-based Predictor } \\ \text { ESO } & \text { Extended State Observer } \\ \text { FSA } & \text { Finite Spectrum Assignment } \\ \text { LHS } & \text { Left-Hand Side } \\ \text { LMI } & \text { Linear Matrix Inequality } \\ \text { LTI } & \text { Linear Time-Invariant } \\ \text { LQR } & \text { Linear Quadratic Regulator } \\ \text { MAGLEV } & \text { Magnetic Levitation } \\ \text { MIMO } & \text { Multiple-Input Multiple-Output } \\ \text { PID } & \text { Proportional Integral Derivative } \\ \text { RHS } & \text { Right-Hand Side } \\ \text { SISO } & \text { Single-Input Single-Output } \\ \text { ZOH } & \text { Zero-Order Hold }\end{array}$



Chapter 1

\section{Introduction}




\subsection{Introduction: robust control of uncertain systems}

A system is said to be uncertain if, with more or less frequency, it manifests behaviors that are relatively different than the ones predicted by its model. The most common sources of uncertainty are: modeling errors; unknown/unmodeled dynamics; changes in the system properties; random interactions with other systems; or changes in the operating conditions.

In this sense, if it is not possible to construct an accurate ${ }^{1}$ model for a system -due to, for example; its complexity, its economical cost, its apparent random behavior or the lack of knowledge about how some parts of the system behaveone is simply forced to assume that the system is relatively uncertain.

Most of the engineering; natural; economical or biological systems are used to be uncertain. Even the most apparently simple ones. For example, let us consider the accelerator pedal in a car and the effect that it has on the car's velocity. This is a rather simple system with one input -pedal acceleratorand one output -car's acceleration. Depicted its conceptual simplicity, the system is still uncertain. Although it is quite sure that pressing the accelerator always generates positive accelerations, it is not so sure how much does in fact the car accelerates. One could try to take the car in order to perform some experiments with the main objective of building a model -i.e. called a nominal model- that quantitatively gives its acceleration in terms of how much the pedal has been pressed. However, the resulting model will be likely to give errors once the experimental conditions are changed. In fact, the acceleration depends -among many other factors- on the number of passengers, on the road slope, on the wind velocity and on its direction. Developing a car-model that considers all these factors becomes, in many cases, excessively complex; so, at the end, the control-designer is simply forced to assume that car acceleration is relatively uncertain with respect to the nominal model that was identified.

\section{The development of the robust control methods (1960-2000)}

These system uncertainties have posed a fundamental question in the control theory, that is: how to design effective controllers for uncertain systems. This issue has been progressively gaining importance since the early beginnings of 1960s; to the point that, during 1970s it generated an important paradigmshift towards the so-called robust control (Petersen et al. 2014; Safonov 2012; Gao 2014; Sariyildiz et al. 2020).

\footnotetext{
${ }^{1} \mathrm{By}$ accurate it is meant a model that is capable of quantitatively predicting the system behavior in all the possible circumstances and operating conditions.
} 
The main idea behind the robust control methods is clear: the system uncertainties cannot be just neglected. Instead, they should be considered -somehow- in the nominal models that are used to design and test controllers.

This design-premise is supported on different researches and experimental results that have revealed that the system uncertainties could have very negative effects in a feedback controlled system if they are not properly considered. One of the first analyses in this line came from Horowitz 1963. He mathematically proved that the system uncertainty is a dominant factor in determining what can be achieved with feedback control. He postulated that there exist a tradeoff between the controller robustness -i.e. the amount of uncertainty in the nominal model that can be tolerated- and its performance.

Other significant results appeared during 1970s. Different works -e.g. (Rosenbrock et al. 1971; Athans 1971; Doyle 1978)- reported strong failures of some optimal control techniques that were caused, mainly, by the system uncertainties. It was proved that an arbitrarily small difference between the real system behavior and its nominal model could be theoretically capable of destabilizing the - popular- LQG controller. Similar problems were experimentally observed during the same decade. Safonov 2012 makes reference to some attempts to apply the LQG controller in a Trident submarine and in a F-8C crusader aircraft. It was reported that the LQG caused the submarine to unexpectedly go to surface in simulations under moderately rough seas; whereas some "weaknesses" of the theory were the main reasons attributed to problems in the F-8C aircraft.

It is said that these failures of the LQG controller during 1970 were the main trigger for all the subsequent developments of robust control techniques (Safonov 2012; Sariyildiz et al. 2020). During 1970-2000 a wide number of papers were published with different methodologies in order to build robust controllers for uncertain systems. The works developed during this period can be categorized into two areas: robust control designs and robust control analyses (Petersen et al. 2014). The first one deals with the problem of designing the "best" controller for a given nominal model with some predefined uncertainty. The second one deals with the companion problem: finding the maximum "amount" of model-uncertainty that can be tolerated by a given controller.

Some noteworthy results developed during this period for robust control analyses are: the multi-variable stability margins (Safonov 1980); the small gaintheorem (Sandberg 1964; Zames 1966); the Kharitonov theorem (Chapellat et al. 1989); the Lyapunov-based robustness analyses (Freeman et al. 2008; Khalil et al. 2002) or the Linear Matrix Inequality (LMI) approach (Scherer 
et al. 2000). These mathematical methods basically allow to check the effects that some type of system uncertainty may have on a given controller.

On the other hand, the most distinguished approaches to design robust controllers are: the $\mathcal{H}_{\infty}, \mathcal{H}_{2}$ or $\mu$-controllers (Doyle et al. 1988; Doyle et al. 1989; Khargonekar et al. 1991); the -recently popular- Disturbance ObserverBased Controllers (DOBCs) and related methods (Ohishi 1983; Sariyildiz et al. 2020; Chen et al. 2016; Schweppe 1968; Johnson 1975; Meditch et al. 1973; Bhattacharyya 1978; Darouach et al. 1994); the Active Disturbance Rejectionbased Control (ADRC) (Han 2009; Wu et al. 2020); the sliding-mode controllers (Edwards et al. 1998); the Disturbance Accommodating Control (DAC) theory (Johnson 1971; Johnson 1986); and the Internal Model-based Control (IMC) (Francis et al. 1976; Garcia et al. 1982). All these results can be employed to design controllers for uncertain dynamic systems. Thus, they share the peculiarity that some type of system uncertainty is explicitly considered during the control-design process.

\section{The robust control methods during the technological revolution (2000-2020)}

Some of these robust control methods have been proved to be quite effective, being widely known today by the successful results that they have achieved in practice. In particular, the DOBCs, the ADRC and the sliding-mode controllers are becoming very popular among scientist as they permit to synthesize high-precision feedback regulators with just very basic system information (Han 2009; Huang et al. 2014; Sariyildiz et al. 2020; Gao 2006; Gao et al. 2001; Chen et al. 2016).

This can be appreciated in Fig. 1.1, which contains the approximate number of new IEEE papers that were published during 2000-2020 containing keywords of the main robust control techniques ${ }^{2}$. It can be seen a significant growth on their use; being remarkable the exponential rise of DOBCs, ADRCs, and sliding mode controllers. Interestingly, the figure illustrates that around the $50 \%$ of the total number of papers referencing to these controllers have been published during the last 5 years. This indicates that, nowadays, these robust controllers are being developed/applied faster than in any other period.

\footnotetext{
${ }^{2}$ The search has been performed by Google Scholar with the following commands: i) ("disturbance observer" OR "Unknown input observer" OR "Extended state observer") source:"IEEE"; ii) ("H infinity" OR "H_\{ $\backslash$ infty\}" OR "H infinite" OR "H/sub infinity") source:"IEEE"; iii)("sliding mode control") source:"IEEE"; iv) ("internal model principle" OR "internal model control" OR "IMC") source:"IEEE"; v) ("active disturbance rejection" OR "ADRC") source:"IEEE"
} 

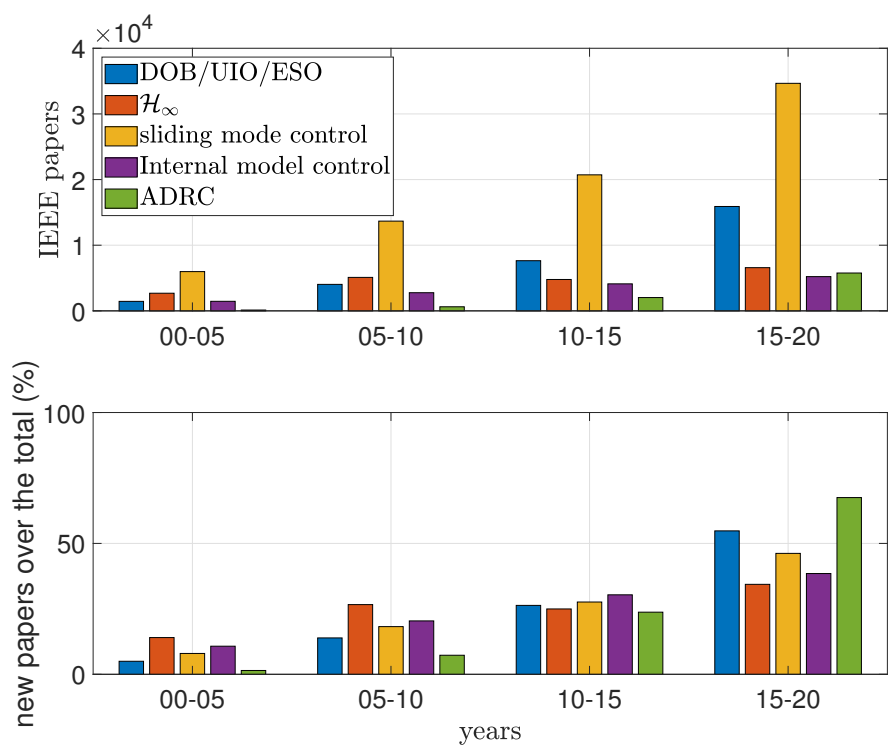

Figure 1.1: Top plot: Approximate number of new IEEE papers -in intervals of 5 yearscontaining keywords related with the different robust control strategies. Bottom plot: (number of new papers)/(total number of papers)· 100 .

Furthermore, if the search is focused on specific application areas, it is found that robust controllers -specifically DOBCs, ADRCs and sliding mode controllers- are being applied in: motion control (Umeno et al. 1991; Choi et al. 1999; Sariyildiz et al. 2015), power electronics (Yokoyama et al. 1994; Jain et al. 2020), fault diagnosis (Wang et al. 1996; Han et al. 2018; Bernardi et al. 2020), automobiles (Bünte et al. 2002; Kadowaki et al. 2002; Fujimoto et al. 2004), underwater vehicles (Peng et al. 2017), networks (Natori et al. 2008), missile seekers (Sadhu et al. 2010), spacecrafts (Liu et al. 2018), UAVs (Castillo et al. 2019a), surgery/rehabilitation robots (Ugurlu et al. 2014), long-distance laser positioning (Deng et al. 2020) and magnetic levitation (Yang et al. 2011); among many others.

In addition, their application to other fields, such as: economics (Bernhard 2003; Hansen et al. 2008), game-theory (Başar et al. 1998) or biotechnology (Sala-Mira et al. 2019; Sanz et al. 2020); is also being explored.

However, despite the positive evolution of these control methods, in many cases scientists are still finding difficulties on how to proceed when designing robust feedback regulators; specially, for relatively complex multi-variable systems 
that are sensitive to external disturbances or uncertain factors (Guo et al. 2014; Sariyildiz et al. 2020; Chen et al. 2016; Lamnabhi-Lagarrigue et al. 2017; Petersen et al. 2014). This is mainly caused by a lack of more generalized -and conceptually simple- robust control-design methods.

\subsection{Contributions of this thesis}

In this thesis, new strategies to design robust Disturbance Observer-Based Controllers (DOBCs) and Predictors (DOBPs) for uncertain dynamic systems are developed.

As mentioned above, the Disturbance Observer (DOB) approach is becoming one of the most popular solutions for dealing with uncertain dynamic systems; mainly, due to its conceptual simplicity. The operating principle of a DOB is rather simple: a nominal-model -normally Linear and Time-Invariant (LTI): $\dot{x}(t)=A x(t)+B_{2} u(t) \in \mathbb{R}^{n}, y(t)=C x(t) \in \mathbb{R}^{p}$ - that was identified for the system is taken and, then, some unknown inputs - called disturbances, $\omega(t)$ - are added to it. This conforms the following LTI disturbed model for the system:

$$
\begin{aligned}
& \dot{x}(t)=A x(t)+B_{2} u(t)+B_{1} \omega(t), \\
& y(t)=C x(t) ;
\end{aligned}
$$

whose chief characteristic is that it includes some extra degrees of freedom -given, precisely, by the disturbances- that easily permit to account for the possible uncertain behaviors of the system with respect to the nominal-model ${ }^{3}$.

The DOB approach makes use of the historical set of sensor readings, $y(t)$, in order to compute estimates of the nominal model-state, $\hat{x}(t)$, as well as the disturbance signals, $\hat{\omega}(t)$, that are affecting to it.

In this sense, the computed estimates, $[\hat{x}(t), \hat{\omega}(t)]$, contain information about the current state of the system according to the LTI disturbed model and the historical set of sensor readings. Due to this property, DOBs are mainly used to for: i) feedback Control -i.e. DOBCs- (Han 2009; Shim et al. 2009; She et al. 2008; Li et al. 2012; Castillo et al. 2018; Khlebnikov 2016); ii) Prediction of the future system-state -i.e. DOBPs- (Sanz et al. 2016b; Castillo et al. 2020b); and iii) Failure Detection -i.e. DOBFDs- (Wang et al. 1996; Han et al. 2018; Bernardi et al. 2020).

New design-methodologies to solve the first two problems are developed in this thesis.

\footnotetext{
${ }^{3}$ More specific details on this are given in Chapter 2
} 


\section{A novel strategy to synthesize DOBCs}

The main problem in DOBC concerns the issue of how to design an appropriate feedback, e.g. $u(t)=K_{x} \hat{x}(t)+K_{\omega} \hat{\omega}(t)$, that sets the control action, $u(t)$, in terms of both the nominal model-state, $\hat{x}(t)$, and its disturbances, $\hat{\omega}(t)$.

Designing this feedback is a crucial step when constructing robust DOBCs for any application, as it mainly defines the closed-loop system behavior. Its design can be relatively easy for models satisfying the so-called matched condition (Chen et al. 2016; Sariyildiz et al. 2020); but -without a proper methodology- it could become considerably complex for multi-variable models with mismatched and/or multiple disturbances. Currently, there is an increasing interest in exploring new solutions to this feedback-design problem, e.g. (Li et al. 2012; Castillo et al. 2018; Guo et al. 2014; Pu et al. 2020; Khlebnikov 2016), as any new strategy may contribute to increase and simplify the applicability of this robust control technique.

This thesis provides a novel approach to this feedback-design problem, which is developed from the optimal control theory. It is shown how DOBCs of the form $u(t)=K_{x} \hat{x}(t)+\sum_{i=0}^{r} K_{\omega, i} \hat{\omega}^{(i)}(t), r \in \mathbb{N}$, can be synthesized with the main objective of approximating the -unrealizable- optimal control signal, $u_{\gamma}(t)$, minimizing a quadratic-cost index subject to the LTI disturbed model.

This novel feedback design strategy contains significant differences -from both conceptual and technical points of view- if compared with the previous methods; which mostly design the DOBCs in order to reject the disturbance signals in some channel -e.g. (Chen et al. 2016; Sariyildiz et al. 2020; Yang et al. 2011; She et al. 2008; Castillo et al. 2018; Li et al. 2012)- or with ellipsoidal Lyapunov-based methods -e.g. (Khlebnikov 2016).

Its major advantage is that, as it is developed from an optimality-based approach, the solution is indistinctly valid for SISO/MIMO models with single/multiple matched/mismatched disturbances. In addition, it offers an intuitive and versatile tuning through the cost-index weighting matrices. 


\section{A novel strategy to synthesize DOBPs}

The main problem in DOBP concerns the issue of how to make use of the estimates $[\hat{x}(t), \hat{\omega}(t)]$ in order to predict the future nominal-state, $\hat{x}\left(t+\Delta_{t}\right)$.

Predicting the value of $\hat{x}\left(t+\Delta_{t}\right)$ is often needed for control purposes. For example, predictions are extensively used for closed-loop input-delay compensation (Manitius et al. 1979; Artstein 1982; Krstic 2008; Krstic 2010; Mazenc et al. 2012; Li et al. 2014); for controlling systems with delays and disturbances (Di Loreto et al. 2005; Sanz et al. 2016b; Furtat et al. 2017; Santos et al. 2018); for model predictive control (Mayne 2014; Binder et al. 2019); and, also, in other applications where estimates of $x\left(t+\Delta_{t}\right)$ may be useful, such as collision avoidance (Polychronopoulos et al. 2007).

To the best of our knowledge, there exist very few results to this prediction problem. The existing ones have been mainly developed within the framework of control of time-delay systems -e.g. (Sanz et al. 2016b; Furtat et al. 2017; Léchappé et al. 2015).

This thesis shows to construct state-predictors of the form $\hat{x}\left(t+\Delta_{t}\right)=\Phi\left(\Delta_{t}\right) \hat{x}(t)+$ $\sum_{i=0}^{r} \Gamma_{\omega, i}\left(\Delta_{t}\right) \hat{\omega}^{(i)}(t)+\sum_{k=0}^{N} \Gamma_{u}\left(\tilde{t}_{k}, \tilde{t}_{k+1}\right) u_{k}$; with the main purpose of approximating the time domain solution of the LTI disturbed model.

\subsection{Background: why this thesis was developed and its main motivations}

Developing new tools to synthesize DOBCs and DOBPs might be very well received by engineers and control practitioners. Specially, if they are valid for multi-variable models with multiple or mismatched disturbances and they offer an intuitive and versatile tuning. Results in this line may contribute to: save both time and resources in what respects to control-design tasks; design DOBCs for a much wider class of systems and scenarios; and to enrich the existing literature about this robust control strategy, which is constantly gaining acceptance.

These are the main motivations that lead us to develop this research. The main purpose of this section is to give a chronological picture of the main events that conducted us to do it. 


\section{Some experimental tests of DOBCs in drones}

By 2015-2016, we were experimentally working with drones. Concretely, on testing and implementing feedback-regulators to stabilize them. Drones are systems that are quite sensitive to some uncertain elements, mainly: wind gusts, load variations, battery discharges or changes in its geometrical distribution. These uncertainties degrade the controller performance in some situations, being even capable of destabilizing the drone itself.

Conventional drone controllers were mostly based on PID regulators; which suppress the effect of the uncertainties by the integral action. Although the integral action is capable of rejecting constant disturbances, it has some disadvantages; such as: oscillatory responses and a non-trivial gain tuning. This caused that the PID controllers failed to achieve a high-precision response in environments with strong disturbances.

Seeking for ways to enhance the drone performance, we got interested on the DOBCs (Chen et al. 2016; Sariyildiz et al. 2020). The current literature was indicating that these controllers had significant capabilities for detecting and compensating the uncertainties that affect to the systems; allowing a highprecision control in situations with high disturbances. In fact, its use was growing fast -refer to Fig. 1.1. So we started to test them.

The results were surprisingly good. A first preliminary result was presented at the IEEE International Conference on Information and Automation (Castillo et al. 2016), and more complete results with experimental demonstrations were latter published in Castillo et al. 2019a -in collaboration with the Zhejiang University, Hangzhou, China. In that work, a DOBC was implemented in an aerial drone. Disturbance signals, $\omega(t)$, were introduced in the nominal model of the drone in order to account for the Coriolis effect and other unknown torques that affect to the drone when flying -such as payloads, wind gusts, etc. A DOB was employed to estimate these unknown disturbance signals, which were then used in a feedback control-law. The results were remarkable. The drone was able to compensate for its own Coriolis effect without none information about its inertia matrix. It was also capable of detecting and compensating for significant changes of weight in some of its arms.

Given that this accurate behaviour had never been achieved by us with other control strategies, it became a good reason to explore other engineering control problems in which DOBCs may have benefits. 


\section{Attempts to design DOB controllers for other systems}

We started by looking at the problem of closed-loop glucose-control for people with type-1 diabetes; e.g. (Tecnodiabetes ai2, UPV; Sanz et al. 2020). The control problem consist in the following: measures of the glucose concentration in blood are taken and it is needed to develop a controller that -based on these measurements- automatically injects insulin to the person if needed. The objective is to maintain the glucose concentration in some safety levels. The uncertainties of this process are mainly related with human behaviors -i.e. unannounced meals and sport activity that cause undesired glucose variations.

Encouraged by the results with drones, we faced this new problem and we attempted to design DOBCs for it. The objective was that the disturbance signals, $\omega(t)$, introduced in the nominal patient-model should detect the situations in which the patient eats something. Then, they could be employed to automatically generate an insulin bolus in order to reduce the increase of glucose after the meal.

A wide number of different DOBC methods in a simulator running with a clinically identified model (Stocker et al. 2006; Kanderian et al. 2009; Kanderian et al. 2012) were tested; including the ADRC approach (Wu et al. 2020; Huang et al. 2014), the GESO-based control (Li et al. 2012), the classical frequency-domain DOBC (Ohishi 1983; Sariyildiz et al. 2020) as well as some other similar approaches reported in (Sariyildiz et al. 2020; Chen et al. 2016). All these DOBCs - which appropriately worked in drones- failed in this new scenario, even in the most simple simulations.

The main problem was that all these DOBCs were mostly designed to achieve a complete rejection of the disturbance signals through the control-input channel. They directly feedback the disturbance estimates in order to cancel for the "real" ones. Achieving a complete disturbance compensation was attainable in drones, but it was physically impossible in this new scenario due to both the system dynamics and the physical limitations of the actuators. These DOBCs were, simply, too aggressive. We then realized that a suitable solution here could be some kind of feedback design-method that allowed to penalize the excessive use of control action at the expense of not compensating the entire disturbances.

Subsequently, we noticed that similar problems were also found by other authors that were dealing with other engineering control problems, such as: MAGnetic LEVitation (MAGLEV) control, permanent magnet synchronous motors or fixed-wing UAVs (Yang et al. 2011; Li et al. 2012). 


\section{Exploring new methods for synthesizing DOBCs}

The lack of feedback design-tools for DOBCs started to be seen as a major problem by us. On the one hand, it was known that this robust control strategy was able to offer very good behaviors in some systems - as showed by our previous experiments with the drones. But, on the other hand, it seemed that the absence of more generalized feedback design-strategies was braking new applications of this technique to other control problems.

We realized that new contributions in this line could be very interesting for both control practitioners and control theorists. Thus, the main research started to focused on this topic, ending with the main results of this thesis. A first preliminary result to the feedback-design problem was proposed in (Castillo et al. 2018) for SISO models and, later, two additional ones -constructed from an optimality-based approach- were developed for MIMO models with possibly multiple disturbances (Castillo et al. 2020c; Castillo et al. 2020d).

After these results, we come to the conclusion that some parts of the technical developments employed to solve the feedback-design problem for DOBCs could be also applied to the prediction problem. This permitted to publish the work (Castillo et al. 2020b), summarizing the main results on DOBPs.

It could be said that the whole process has been relatively long and it has required a detailed study of the classical and the modern control theories in its various branches. The final results are summarized here in a constructive way, starting from what we understand to be the most basic definitions/ideas and developing over them.

\subsection{Chapters organization}

Fig. 1.2 depicts a diagram of the remaining chapters; indicating how this thesis could be read.

Chapter 2 contains the main definitions and the problem statement. Its main purposes are: i) to better illustrate the usefulness of the LTI disturbed models for representing systems with uncertain behaviors; ii) to define more clearly what a DOB is; iii) to introduce the generalized four-block control-design problem for LTI disturbed models; iv) to define the optimal controller -namely, $u_{\gamma^{-}}$ and the state-predictor $-x\left(t+\Delta_{t}\right)$ - that will be considered as the basis for synthesizing the proposed DOBCs and DOBPs; and $\mathbf{v}$ ) to establish some relationships with other classical and recent control strategies. 


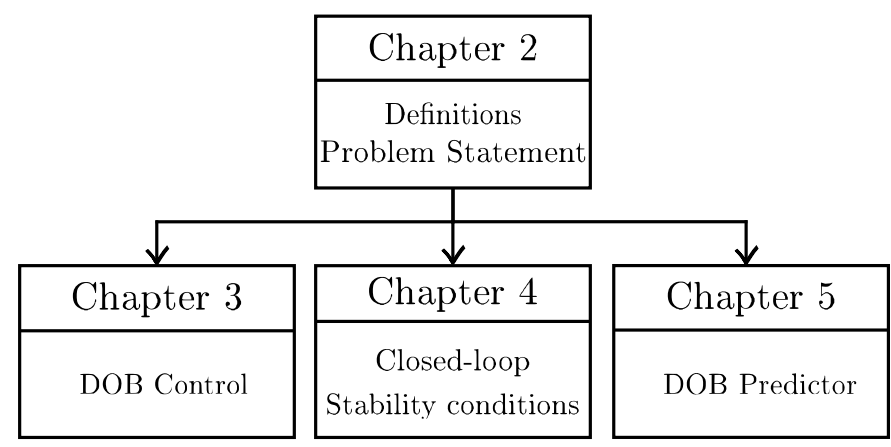

Figure 1.2: Chapter organization

Chapter 3 shows how to synthesize DOBCs of the form $u(t)=K_{x} \hat{x}(t)+$ $\sum_{i=0}^{r} K_{\omega, i} \hat{\omega}^{(i)}(t), r \in \mathbb{N}$, that approximately implement the optimal controller $u_{\gamma}$.

Chapter 4 includes some closed-loop analyses and stability conditions for the DOBCs.

Chapter 5 shows how to synthesize DOBPs of the form $\hat{x}\left(t+\Delta_{t}\right)=\Phi\left(\Delta_{t}\right) \hat{x}(t)+$ $\sum_{i=0}^{r} \Gamma_{\omega, i}\left(\Delta_{t}\right) \hat{\omega}^{(i)}(t)+\sum_{k=0}^{N} \Gamma_{u}\left(\tilde{t}_{k}, \tilde{t}_{k+1}\right) u_{k}$, that approximate the model's solution $x\left(t+\Delta_{t}\right)$.

Chapter 2 should be firstly read. After it, one can indistinctly jump to Chapter 3,4 or 5; as they are mostly independent among them.

\section{Relationships between publications and chapters}

Different publications have been written during this project. Some of them are already published, i.e. (Castillo et al. 2016; Castillo et al. 2017; Castillo et al. 2018; Castillo et al. 2019a; Castillo et al. 2019b; Castillo et al. 2020b; Castillo et al. 2020d); and some others are currently under editorial review (Castillo et al. 2020a; Castillo et al. 2020c).

The chapters of this document can be related to some of the published papers. Concretely, the results of Chapter 4 are mainly the ones in Castillo et al. 2019b -being also related with the work Castillo et al. 2017. The content of Chapter 5 is published in Castillo et al. 2020b. Chapter 3 has been sent for publication and it is under editorial review in Castillo et al. 2020c. 


\section{Chapter 2}

\section{Problem statement}

The main definitions and the problem statement is contained in this chapter.

The main purposes are: i) to give insights into the LTI disturbed models and their usefulness for representing systems with uncertain behaviors; ii) to define what a DOB is; iii) to introduce the generalized four-block control-design problem for LTI disturbed models and; iv) to define the optimal controller -namely, $u_{\gamma}-$ and the state-predictor $-x\left(t+\Delta_{t}\right)$ - that will be considered as the basis for synthesizing DOBCs and DOBPs.

In addition, some relationships with other classical and recent control strategies are established. 


\subsection{LTI disturbed models: a simple framework to deal with uncertain systems}

As mentioned in Sec. 1.2, one of the key elements of the DOB methods is that they add some unknown inputs -called disturbances- to the nominal models in order to account for the possible uncertain behaviors. In this section, some depeer insights into this method and the resulting LTI disturbed models are given.

\section{Nominal deterministic models and the inherent complexity on making them accurate}

For illustrative purposes, let us consider the following nominal model for the vertical position of an aircraft:

$$
\begin{aligned}
& \dot{p_{z}}(t)=v_{z}(t), \\
& \dot{v}_{z}(t)=m u(t)-g ;
\end{aligned}
$$

where $g=9.81\left(\mathrm{~m} / \mathrm{s}^{2}\right)$ is the gravity constant; $u(t)(\mathrm{N})$ is the vertical propulsion force; $m(\mathrm{~kg})$ is the aircraft mass; $v_{z}(t)(\mathrm{m} / \mathrm{s})$ is the vertical velocity and $p_{z}(t)(\mathrm{m})$ is the vertical position.

This model mostly comes from the Newtonian mechanics. Let us contemplate the following questions: i) Is this model capable of accurately representing the real altitude of an aircraft?; and, if not, ii) which modifications should be done? Questions regarding the accuracy of a model are relatively important from a control-design perspective as a huge number of mathematical-tools to design controllers which, at the end, require mathematical models have been developed within the framework of the control theory. In this sense, the more accurate the model is, the better control results will be achieved by these tools.

In order to answer both questions, one should firstly define a clear experimental procedure that permits to evaluate the accuracy of the model. Let us suggest the following one: install a high-precision altitude sensor on the aircraft so that its current altitude, $y(t)$, is known. Then, put the aircraft on fly-for example, by manually driving the force $u(t)$ - and compare the measurements given by the sensor, $y(t)$, with the ones predicted by the model, $p_{z}(t)$. If the model is accurate, then, it should be satisfied that $y(t) \approx p_{z}(t)$. In other words: an accurate model is capable of predicting the sensor readings.

It may be noted that, without an experimental procedure like this one, which necessarily implies to install sensors and compare the sensors readings with 
the model output, is not possible to make statements about the accuracy of some hypothetical model. Thus, an "accurate model" simply means that it is capable of predicting the observed/measured data of the system.

Let us consider that an experiment like this one is performed in a real scenario with an aircraft and the model (2.1). In all likelihood, it would be found that the altitude measures, $y(t)$, differ from the ones, $p_{z}(t)$, predicted by $(2.1)$. Hence, the answer to $\mathbf{i})$ would be negative in most of the experiments. The reason is that, from previous experiences, it is known that the model (2.1) is neglecting many other factors that actually affect the aircraft -such as the wind force, the air density, how the propulsion force $u(t)$ is being generated, etc. All these spurned factors constitute the main source of the discrepancies between $p_{z}(t)$ and $y(t)$.

In this sense, one could think that enhancing the accuracy of (2.1) goes through modeling new elements, such as: i) the aircraft aerodynamics, ii) the wind force, or iii) how the vertical force $u(t)$ is being generated. The modeling process could be considered as concluded whenever some equations -say $\dot{p}_{z}(t)=$ $f_{1}\left(v_{z}(t)\right), \dot{v}_{z}(t)=f_{2}\left(v_{z}(t), u(t)\right)$, which include information about the aircraft aerodynamics, wind velocity, air pressure, etc- are found such that, for any flight that is done, the altitude predicted by the model matches with the sensor readings; i.e. $y(t) \approx p_{z}(t)$.

This pair of equations represents the well-known two-equation model -i.e. the system dynamics plus the measurement equation- that is extensively employed within the classical control theory. In this case, it would result in:

$$
\begin{aligned}
& \dot{p}_{z}(t)=f_{1}\left(v_{z}(t)\right), \\
& \dot{v}_{z}(t)=f_{2}\left(v_{z}(t), u(t)\right), \\
& y(t)=p_{z}(t) .
\end{aligned}
$$

A generalized dynamical model takes the form $\dot{x}(t)=f(x(t), u(t)) \in \mathbb{R}^{n}$, $y(t)=h(x(t), u(t)) \in \mathbb{R}^{p}$; or, if it is linear:

$$
\begin{aligned}
& \dot{x}(t)=A x(t)+B u(t), \\
& y(t)=C x(t)+D u(t) ;
\end{aligned}
$$

where $x(t) \in \mathbb{R}^{n}$ is an internal variable representing the nominal model-state; $u(t) \in \mathbb{R}^{m}$, is the control input; and $y(t) \in \mathbb{R}^{p}$, the sensor readings.

Certainly, a major disadvantage of these models is the strong complexity on making them accurate. Finding some deterministic function, $f(\cdot)$-or matrices 
$(A, B, C, D)$ - which, simply by receiving the control inputs, is capable of predicting the aircraft altitude -that is, the measurement equation holds for all the time and all the possible circumstances- would never be simple.

\section{LTI disturbed models: an alternative way to mathematically represent the systems}

In order to solve this disadvantage, let us now consider the following slightly modified LTI disturbed model:

$$
\begin{aligned}
& \dot{p}_{z}(t)=v_{z}(t), \\
& \dot{v}_{z}(t)=m u(t)-g+\omega(t) ;
\end{aligned}
$$

where $\omega(t)$ is an unknown input -called disturbance- that can take, a priory, any waveform without none specific physical meaning.

The main difference with respect to (2.1) is that (2.5) contains the unknown input, $\omega(t)$. By this reason -in contrast to (2.1)- the output of the model (2.5) is not entirely defined. Now, for the same control input $u(t)$ and the same initial states, $p_{z}\left(t_{0}\right), v_{z}\left(t_{0}\right)$; model (2.5) can generate a whole set of different outputs, one for each $\omega(t)$.

Thence, if we refer to the same question regarding the capability of (2.5) for representing the real aircraft altitude, it can be easily concluded that the answer is now positive in most cases. In order to verify it, a similar experiment could be done: the aircraft is put on fly and all the data given by the altitude sensor, $y(t)$, together with the control inputs, $u(t)$, are recorded. After the fly, the data could be taken and it could be corroborated that -in fact- there exists one function, $\omega(t)$, so that the altitude given by (2.5) matches with the measurements provided by the sensors, i.e. $p_{z}(t) \approx y(t)$.

The experiment could be repeated again, but this time performing new maneuvers and flying with other environmental conditions. After this second flight, the new recorded data could be taken and it could be corroborated that there exists another different function, i.e. $\omega_{2}(t)$; so that the output of the same model (2.5) matches with the altitude measurements. The experiment could be repeated again and again. With every flight that is done, a new -and probably different- function $\omega(t)$ so that $y(t) \approx p_{z}(t)$ will be found. In fact, under some non-strong assumptions over the sensor readings, $y(t)$, it can be proved that there always exist some $\omega(t)$ so that $y(t)=p_{z}(t)$. 
This viewpoint - which is rather different than the previous one- is also mathematically represented by the well-known two-equations model:

$$
\begin{aligned}
& \dot{p}_{z}(t)=v_{z}(t), \\
& \dot{v}_{z}(t)=m u(t)-g+\omega(t), \\
& y(t)=p_{z}(t) .
\end{aligned}
$$

A generalized LTI disturbed model takes the form:

$$
\begin{aligned}
& \dot{x}(t)=A x(t)+B_{2} u(t)+B_{1} \omega(t) \in \mathbb{R}^{n} \\
& y(t)=C x(t)+D_{2} u(t)+D_{1} \omega(t) \in \mathbb{R}^{p}
\end{aligned}
$$

where the first equation represents the system dynamics - with its respective unknown disturbance inputs- while the second one represents the measurement equation.

\section{Main differences between both modeling approaches}

There are two substantial differences that should be established. The first -and probably the most important- one is about the measurement equations in (2.4) and (2.7). In the case of the nominal model (2.4), the measurement equation, more than a strict equality, should be understood as something that may hold only if the model is sufficiently accurate. This equality is a consequence of the model accuracy as it is comparing two things that are originally different, say: the sensor readings with the deterministic and unique solution of the model. Therefore, the measurement equation will not hold if the model is inaccurate.

However, in the case disturbed models (2.7), the measurement equation is forced to be a strict equality. This can be done because, now, the model includes some undefined functions, $\omega(t)$, whose value can be freely chosen in order to force the measurement equation as an strict equality ${ }^{1}$. So, from a mathematical perspective, $\omega(t)$ gives an extra degree of freedom that can be used to strictly force the measurement equation. And, from a control perspective, this is equivalent to say that our trust in the sensor readings is greater than in the model and, consequently, we construct an uncertain model that continuously adapts itself to the sensor readings. In other words, it is being assumed that the sensor

\footnotetext{
${ }^{1}$ Clearly, there is an inherent assumption here which is that "there exist some $\omega(t)$ so that the measurement equation is satisfied". This does not necessarily hold in practice if the disturbances are introduced wrongly in the models, if the models contain excessive errors, or if the environmental circumstances dramatically change. Models should be constructed with prior information about the process and the environment; and the disturbances should be placed correctly in the models. In any case, this assumption can be checked experimentally.
} 


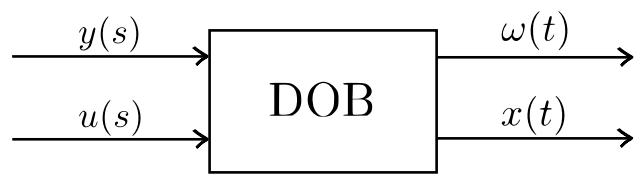

Figure 2.1: DOB block diagram. It receives as inputs the measured information, $y(s)$, and the control input, $u(s)$, with $s \in\left[t_{0}, t\right], t_{0}<t$; and it provides estimates of $\omega(t)$ and $x(t)$.

readings are generated by an uncertain system of the form (2.7), whose degree of freedom is $\omega(t)$.

The second difference lie in the non-defined nature of the disturbed models. Disturbed models are just well-defined up to the current time $t$-in the sense that, for a given initial states $p_{z}\left(t_{0}\right), v_{z}\left(t_{0}\right), t_{0}<t$, a given control input, $u(s)$, and a given sensor readings, $y(s), s \in\left(-t_{0}, t\right]$; one could find a disturbance signal, $\omega(s)$, and a nominal system states, $p_{z}(s), v_{z}(s)$, so that $(2.7)$ holds for all $s \in\left(-t_{0}, t\right]$. However, the future states and the future disturbance signals will never be known until time moves forward and new sensor readings are available. This is not the case with undisturbed models; which just by defining the initial states and the control input, its solution can be integrated for all the time.

\subsection{DOBs: numerical methods for estimating disturbance signals}

A DOB is an algorithm -normally a recursive/differential equation- whose inputs are the sensor readings, $y(s)$, and the control signals $u(s), s \in\left[t_{0}, t\right]$ and it provides as an output real-time estimates, $\hat{x}(t), \hat{\omega}(t)$, of the systemstate, $x(t)$, and its disturbance signals, $\omega(t)$. The estimates are computed under the assumption that the sensor readings $y(t)$ are generated by an hypothetical LTI disturbed model of the form (2.7).

In this sense, a DOB gives useful information in real-time about the state of the system according the model and the historical set of sensor readings. By this reason, the estimated pair $[\hat{x}(t), \hat{\omega}(t)]$ has been successfully employed for feedback control -i.e. DOBC- (Han 2009; Shim et al. 2009; She et al. 2008; Li et al. 2012; Castillo et al. 2018; Khlebnikov 2016); for failure detection -i.e. DOBFD- (Wang et al. 1996; Han et al. 2018; Bernardi et al. 2020); and to make predictions of the future system-state -i.e. DOBP- (Sanz et al. 2016b; Castillo et al. 2020b). 


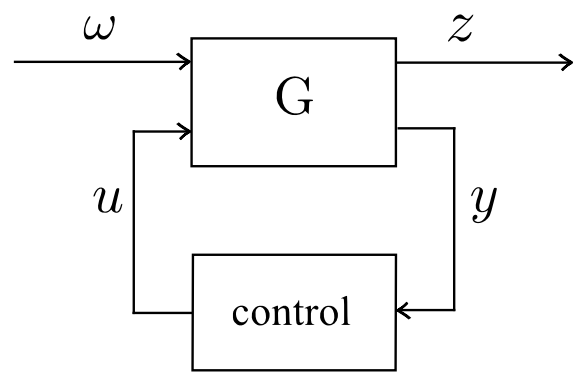

Figure 2.2: The four-block control problem.

In reference to this, during the last decades an important number of DOB algorithms have been developed. Some prominent examples are the ones by Meditch et al. 1973; Bhattacharyya 1978; Darouach et al. 1994; Corless et al. 1998; Xiong et al. 2003; Chang 2006; Kim et al. 2010; Chen et al. 2016; Chakrabarty et al. 2017; Su et al. 2018.

\subsection{Definition of the optimal controller, $u_{\gamma}$, and the state-predictor, $x\left(t+\Delta_{t}\right)$}

After these introductory sections, we are in position to set the things up and present the main optimal controller -namely, $u_{\gamma^{-}}$that will be considered as the basis for synthesizing new DOBCs; as well as the state-predictor -namely, $x\left(t+\Delta_{t}\right)$ - that will be considered as the basis to construct DOBPs.

\section{The "standard" four-block control problem for LTI disturbed models}

Figure 2.2 represents the so-called four-block control problem; a generalized control formulation widely employed within the robust control theory (Petersen et al. 2014; Åström et al. 2014; Doyle et al. 1989; Tadmor 1990).

The block $G$ represents the system to be controlled, which is assumed to be given by the following LTI disturbed model:

$$
G \triangleq\left\{\begin{array}{l}
\dot{x}(t)=A x(t)+B_{2} u(t)+B_{1} \omega(t), \\
y(t)=C_{2} x(t), \\
z(t)=C_{1} x(t),
\end{array}\right.
$$

being $x(t) \in \mathbb{R}^{n}$ an internal variable representing the nominal system-state; $u(t) \in \mathbb{R}^{m}$, the control inputs; $\omega(t) \in \mathbb{R}^{q}$, the unknown disturbance inputs; 
$y(t) \in \mathbb{R}^{p}$, the sensor readings; $z(t) \in \mathbb{R}^{r}$, the controlled variable; and $A, B_{1}$, $B_{2}, C_{1}, C_{2}$, nominal matrices of appropriate dimensions, which define the main interactions between the model variables.

The model $G$ contains two inputs -i.e. the control input, $u(t)$, and the unknown disturbances, $\omega(t)$ - and two outputs -i.e. the sensor readings, $y(t)$, and the controlled variable, $z(t)$. The disturbances, the sensor readings and the control inputs should be understood as described in Sec. 2.1. The role of the controller is to take the sensor readings, $y(t)$, in order to generate a control input, $u(t)$, that maintains the controlled variable, $z(t)$, close to zero ${ }^{2}$.

This constitutes the so-called four-block control-design problem, which is a quite versatile framework to design regulators. Control problems that could be radically different in nature may be equivalently represented by Fig. 2.2 and Eq. (2.8). Some examples will be given through the document, which includes simulations for MAGLEV and glucose control for type-1 diabetic people. Demonstrations of how to design collision avoidance controllers in uncertain environments have been also developed within this framework and experimentally tested with successful results -refer to the conclusions of the document. However, the scope of applicability is even larger; models like (2.8) are employed, not only for representing physical or engineering processes, but also for biological and economic systems (Kanderian et al. 2009; Hansen et al. 2008; Albertos et al. 2010). This framework has also been used in game-theory (Başar et al. 1998).

\section{Definition of the optimal controller $u_{\gamma}$}

The optimal control signal to be considered is:

$$
\begin{aligned}
u_{\gamma}(t) \triangleq & \arg \min _{\forall u} \int_{t}^{\infty}\left[z(s)^{T} z(s)+\gamma u^{T}(s) R u(s)\right] d s \\
& \text { s.t. Model }(2.8)
\end{aligned}
$$

being $\gamma>0$ and $R>0$. Without loss of generality, $R$ can be assumed to be normalized to $\|R\|=1$ - as it is being scaled by $\gamma$.

Controller (2.9) reads as follows: from all the possible control signals, $u$, is chosen the one -i.e. $u_{\gamma}$ - that minimizes $\int_{t}^{\infty} z(s)^{T} z(s)+\gamma u^{T}(s) R u(s) d s$; subject to the model-dynamics $(2.8)$.

\footnotetext{
${ }^{2}$ Many control problems, such as: stabilization, reference tracking or trajectory tracking can be reduced to maintain some internal variable close to zero. This variable is normally defined during the modeling process by an appropriate choice of the internal states $x(t)$.
} 
The tuning parameters $R, \gamma$, can be set to define how much control action is used for control purposes. As $\gamma \rightarrow 0$; the controller minimizes $\int_{t}^{\infty} z(s)^{T} z(s) d s$. This implies -if possible- that $z(t) \rightarrow 0$; which is the desired objective. However, for $\gamma \rightarrow \infty$, the controller tries to minimize $\int_{t}^{\infty} \gamma u^{T}(s) R u(s) d s$; which leads to the trivial solution $u(t)=0$; i.e. there is no control because the cost index mainly penalizes the use of control action. For intermediate values, the controller will drive $z(t) \rightarrow 0$ but without wasting excessive control action.

Eqs. (2.8)-(2.9), together with the interpretation of the disturbance signals given in Sec. 2.1, represent the main basis over which the new DOBCs will be developed. Chapter 3 is mainly focused on how to construct DOBCs that approximately implement, at each time-instant, the optimal control signal $u_{\gamma}$; while Chapter 4 is focused on analyzing its closed-loop stability.

\section{Definition of the state-predictor $x\left(t+\Delta_{t}\right)$}

The state-predictor to be considered is:

$x\left(t+\Delta_{t}\right)=e^{A T} x(t)+\int_{t}^{t+\Delta_{t}} e^{A\left(t+\Delta_{t}-s\right)} B_{u} u(s) d s+\int_{t}^{t+\Delta_{t}} e^{A\left(t+\Delta_{t}-s\right)} B_{\omega} \omega(s) d s ;$

which corresponds to the time-domain solution of (2.8).

Predicting the future state, $x\left(t+\Delta_{t}\right), \Delta_{t} \geq 0$, is often needed for control purposes. For example, predictions are extensively used for closed-loop input-delay compensation by (Manitius et al. 1979; Artstein 1982; Krstic 2010; Mazenc et al. 2012; Li et al. 2014); for controlling systems with delays and disturbances (Di Loreto et al. 2005; Sanz et al. 2016b; Furtat et al. 2017; Santos et al. 2018); in model predictive control (Mayne 2014; Binder et al. 2019); and, also, in other applications where estimates of $x\left(t+\Delta_{t}\right)$ may be useful, such as collision avoidance (Polychronopoulos et al. 2007; Castillo et al. 2020a).

However, as Eq. (2.10) highlights, whether the model contains unknown disturbances, the future nominal-state is dependent on the forthcoming disturbance; which, as mentioned in Sec. 2.1, cannot be known at the current time - i.e. we should wait until the future sensor readings in order to determine the future disturbance signals. By this reason, making predictions of the future nominal-state is not straightforward and -indeed- there are scarce contributions provided to this issue.

Chapter 5 shows how Eq. (2.10) can be approximated by disturbance observers; providing a new procedure to synthesize DOBPs. 


\subsection{Relationships of $u_{\gamma}$ with other controllers}

At this point, it is interesting to establish relationships of the optimal controller being considered -i.e. $u_{\gamma}(2.9)$ - with respect to other controller definitions that already exist in the literature. These relationships should be seen as an important motivation for the choice of $u_{\gamma}$ as a base-controller.

\section{Relationships with the Linear Quadratic Regulator (LQR)}

Of special interest is the relationship of $u_{\gamma}$ with respect to the famous Linear Quadratic Regulator (LQR). In fact, if the system-model (2.8) is considered without disturbance inputs -i.e. $\omega=0$ - then, $u_{\gamma}$ reduces to the definition of classical LQR, whose realization is given by:

$$
u_{L Q R}(t)=K_{x} x(t)
$$

with $K_{x} \triangleq-(\gamma R)^{-1} B_{2}^{T} S_{T}$, being $S_{T}$ the solution to the ARE: $A^{T} S_{T}+S_{T} A-$ $S_{T} B_{2}(\gamma R)^{-1} B_{2}^{T} S_{T}+C_{1}^{T} C_{1}=0$.

Thus, for null disturbance signals, it should be expected that the mathematical expression of $u_{\gamma}$ reduces to (2.11). This will be corroborated in Chapter 3.

For non-null disturbances, controller $u_{\gamma}$ could be interpreted as a "robustified" version of the classical LQR; or as a $\mathrm{LQR}$ for uncertain or disturbed systems.

\section{Relationships with the $\mathcal{H}_{\infty}$ control and related MinMax strategies}

Controller $u_{\gamma}$ can be also related to the $\mathcal{H}_{\infty}$ control and related MinMax formulations. These strategies define a robust controller by considering a differential game of the form:

$$
\begin{aligned}
& \min _{u} \max _{\forall \omega \in \mathcal{L}_{2}} \int_{t_{0}}^{\infty}\left[z(s)^{T} z(s)+\gamma u^{T}(s) R u(s)-\delta \omega(t)^{T} \omega(t)\right] d s, \\
& \text { s.t. Eq.(2.8); }
\end{aligned}
$$

a problem that is equivalent to the minimization of the following upper bound:

$$
\frac{\int_{t_{0}}^{\infty}\left[z(s)^{T} z(s)+\gamma u^{T}(s) R u(s)\right] d s}{\int_{t_{0}}^{\infty} \omega^{T}(s) \omega(s) d s}<\delta ;
$$

for any $\omega \in \mathcal{L}_{2}$ and subject to the system dynamics. 
In this sense, it can be seen that the $\mathcal{H}_{\infty}$ control approach minimizes (2.9) under the assumption that the disturbances actively try to maximize it. This conducts to a MinMax optimization problem -such as (2.12)-for which there is plenty of literature about how to solve it, e.g. (Doyle et al. 1989; Tadmor 1990; Gahinet et al. 1994; Khargonekar et al. 1991; Iwasaki et al. 1994; Mahmoud et al. 1996; Zhou et al. 1996; Petersen et al. 2014).

Note that the $\mathcal{H}_{\infty}$ approach represents a worst-case based design. Hence, it imposes a strong assumption on the disturbance behavior that does not necessarily hold in practice -i.e. that it will actively try to maximize ${ }^{3}$ the cost (2.9). If compared with it, the controller $u_{\gamma}$ avoids the "Max" part. In this sense, it does not impose none prior assumption on the behavior of $\omega(t)$.

\section{Relationships with preview control}

Solving problems of the form (2.9) for scenarios where $\omega$ is totally known -meaning that we know its past, present and future values- has been coined as "preview" control in the classical literature (Tomizuka 1974; Tomizuka 1975). The term preview alludes to the fact that it is needed to anticipate the future evolution of $\omega(t)$ in order to implement the controller.

Although -with the definition of disturbances in Sec. 2.1- it is senseless to consider that the disturbances as known, as well as, to consider its future values as known; the preview control has found its applications for trajectory tracking (Tomizuka 1975); where $\omega$ is not understood as a disturbance in the same sense as in this work, but it is seen as a known exogenous term which depends on the trajectory to be followed. The main motivation behind preview control, as stated by Tomizuka 1974, is to find ways to effectively make use of the trajectory information in order to design optimized controllers.

This assessment directly translates to one of the main motivations behind $u_{\gamma}$ and its approximation by DOBCs -i.e. to effectively make use of the estimated disturbance information for an optimized control design.

\footnotetext{
${ }^{3}$ This is incompatible with the ideas of Sec. 2.1 , where disturbances are just some unknown functions that are included in the models in order to strictly guarantee the measurement equation. At first, they will not try to disturb the system in none predefined way. This MinMax nature of the $\mathcal{H}_{\infty}$ control is the main reason for which is attributed its excessive conservativeness.
} 


\section{Relationships with the DAC theory}

Similarly, the DAC theory of Johnson -e.g. (Johnson 1971; Johnson 1986)also deals with controllers of the form (2.9).

Johnson considered that, if some efforts are put on modeling the disturbances -i.e. to express $\omega(t)$ as the output of an exogenous system driven by unknown impulse inputs- then, the Eq. (2.8) could be augmented with the disturbance model, resulting in a system with a non-controllable part. By proceeding in this way, a problem of the form (2.9) could be solved and implemented in practice without excessive difficulties.

However, the need of modeling the disturbances is a major drawback of the DAC theory. Although it is theoretically possible -as, at the end, disturbances are nothing more than modeling errors or interconnections with other systems (Albertos et al. 2010)-, if something characterizes them is the complexity to understand and to model its nature -as highlighted in Sec. 2.1.

\section{Relationships with the feedforward-based control strategies}

If one refers to the recent literature on control design-strategies for disturbed systems, it may be seen that a popular approach is the use of DOBs in combination with feedforward strategies (Chen et al. 2016; Sariyildiz et al. 2020).

These control-methods basically split the control action into to terms: $u=$ $u_{f b}(x)+u_{f f}(\omega)$; being $u_{f b}(x), u_{f f}(\omega)$, a state feedback and disturbance feedforward terms, respectively. The state-feedback, $u_{f b}(x)$, is normally designed for system stabilization or reference tracking, while the disturbance-feedforward, $u_{f f}(\omega)$, is designed for disturbance rejection. In the case of unknown disturbance signals, the term $u_{f f}(\omega)$ is approximately implemented by DOBs.

In general, one can find a very large number of control results based on this splitting policy, (e.g. Khlebnikov 2016; Guo et al. 2005; Shim et al. 2009; She et al. 2008; Jingqing 1995; Zhong et al. 2004; Ohishi et al. 1987); as well as an extensive number of practical applications that employ it (e.g. Oh et al. 1999; Castillo et al. 2019a; Zhou et al. 2014).

Interestingly, it will be found that the resulting mathematical expression of $u_{\gamma}$ also takes the form $u_{\gamma}=u_{f b}(x)+u_{f f}(\omega)$. However, in contrast to all these previous works, the controller $u_{\gamma}$ does not assume beforehand this composite structure. Rather, the feedback and feedforward terms appear in a natural way; being something inherent of the mathematical formulation of the control- 
problem itself. In addition, the feed-forward term, $u_{f f}(\omega)$, will be found to be rather different than the ones normally employed in the current literature.

\subsection{Conclusions}

The main concepts over which the DOBCs and DOBPs will be built have been defined in this chapter. Concretely, the usefulness of the LTI models and the DOBs for dealing systems with uncertain or complex behaviors has been firstly defined. Then, the generalized four-block control-design problem for LTI disturbed models has been introduced and the main optimal controller, $u_{\gamma}$, and state-predictor, $x\left(t+\Delta_{t}\right)$, that will be considered as a basis to synthesize the DOBCs and DOBPs have been introduced.

Some relationships of the considered controller, $u_{\gamma}$, with respect to other popular control definitions have been also established.

The remain chapters are focused on finding the mathematical expression of $u_{\gamma}$ as well as ways to approximately implement it by DOBCs. Also, it will be shown how $x\left(t+\Delta_{t}\right)$ can be estimated through disturbance observers. 



\section{Chapter 3}

\section{DOBC: Optimality-based design, $u_{\gamma}(t)$}

In this chapter, the synthesis of DOBCs of the form $u(t)=$ $K_{x} \hat{x}(t)+\sum_{i=0}^{r} K_{\omega, i} \hat{\omega}^{(i)}(t), r \in \mathbb{N}$, that approximately implement the optimal controller $u_{\gamma}(t)-E q$. (2.9) - is developed. Comparisons with other DOBC designs are established through numerical simulations for magnetic levitation and glucose control for type-1 diabetes.

Part of the content of this chapter is under editorial review. 


\subsection{Introduction}

In this chapter, it is shown how to synthesize DOBCs of the form $u(t)=$ $K_{x} \hat{x}(t)+\sum_{i=0}^{r} K_{\omega, i} \hat{\omega}^{(i)}(t)$ that approximately implement the optimal controller $u_{\gamma}(t)$-Eq. (2.9)-. To this end, the analytical expression of $u_{\gamma}(t)$ is firstly developed and, then, it is provided the manner in which it can be approximated by these type of DOBCs.

Under the umbrella of the optimal control theory, an entire mathematical apparatus in order to explicitly solve problems similar to (2.9) has been developed. Thus, the way to proceed is: i) to reformulate problem (2.9) into an equivalent finite-horizon one, which can be explicitly solved by the standard tools of optimal control; ii) to invoke the optimal control theory to provide with the conditions that $u_{\gamma}(t)$ may satisfy in order to minimize $(2.9)$; iii) to re-express the minimizing conditions as an analytical control-law of the form $u_{\gamma}(t)=$ $K_{x} x(t)+u_{f f}(\omega)$, being $u_{f f}(\omega)$ a functional depending on the future disturbance signals; iv) to show how this -unrealizable- control-law can be approximately implemented by DOBCs of the form $u(t)=K_{x} \hat{x}(t)+\sum_{i=0}^{r} K_{\omega, i} \hat{\omega}^{(i)}(t)$.

The main contribution of this chapter is featured in the iii) and iv) points. To the best of our knowledge, this is the first methodology enabling an approximate implementation of the unrealizable optimal controller (2.9) by DOBCs. In addition to this, the implementation errors will be mathematically defined and upper bounded.

\section{Main assumptions}

Throughout this chapter, the next assumptions are considered:

Assumption 3.1. $\left(A, B_{2}\right)$ is controllable and $\left(A, C_{1}\right)$ is detectable.

Assumption 3.2. The disturbance signal, $\omega$, is $(r+1)$-times continuously differentiable, with $\left\|\omega^{(r+1)}(t)\right\|<\epsilon_{r+1}$.

Asm. 3.1 is standard for controller design, as it guarantees the existence of a unique solution to the Algebraic Riccati Equation (ARE) (Lewis et al. 2012).

Asm. 3.2 refers to disturbance signals that exhibit a certain degree of differentiability or continuity/regularity. Such property allows to predict its waveform over relatively short time-horizons. In general, disturbances of this kind widely appear in practice, coming from different sources, such as: external forces, torques, friction, set-point/servo tracking commands, actuators failures or modeling errors (Johnson 1986; Gao 2014). 


\subsection{Mathematical expression of $u_{\gamma}(t)$}

The analytical expression of $u_{\gamma}(t)$, in (2.9), is developed in this section.

\section{Minimizing conditions for the finite-horizon case}

The problem (2.9) is firstly re-formulated as a finite-horizon optimal-control problem with a fixed terminal cost:

$$
\begin{aligned}
u_{\gamma} \triangleq & \arg \min _{\forall u} x^{T}(T) S_{T} x(T)+\int_{t}^{T}\left[z(s)^{T} z(s)+\gamma u^{T}(s) R u(s)\right] d s, \\
& \text { s.t. Eq.(2.8); }
\end{aligned}
$$

where $[t, T]$ represents the control-interval and $S_{T}$ is restricted to be the solution to the Algebraic Ricatti Equation (ARE):

$$
A^{T} S_{T}+S_{T} A-S_{T} B_{2}(\gamma R)^{-1} B_{2}^{T} S_{T}+C_{1}^{T} C_{1}=0
$$

which, by Asm. 3.1, exists and is unique.

For any $S_{T}$, Problem (3.1) is equivalent to (2.9) as $T \rightarrow \infty$ (Lewis et al. 2012). Thus, the solution for the finite-horizon case can be taken as an starting point and, then, it can be done $T \rightarrow \infty$ in order to get the solution to (2.9).

From the standard theory of the calculus of variations, it follows that the control signal minimizing (3.1) is given by (Lewis et al. 2012; Gelfand et al. 2000):

$$
u_{\gamma}(s)=-(\gamma R)^{-1} B_{2}^{T}\left[S(s) x_{\gamma}(s)+v(s)\right], s \in[t, T)
$$

with $S(s)$ and $v(s)$ satisfying

$$
\begin{aligned}
& -\dot{S}(s)=A^{T} S(s)+S(s) A-S(s) B_{2}(\gamma R)^{-1} B_{2}^{T} S(s)+C_{1}^{T} C_{1}, \\
& -\dot{v}(s)=\left[A-B_{2}(\gamma R)^{-1} B_{2}^{T} S(s)\right]^{T} v(s)+S(s) B_{1} \omega(s)
\end{aligned}
$$

with terminal conditions $S(T)=S_{T}$ and $v(T)=0$; and $x_{\gamma}(s)$ being the statetrajectory that, starting from $x_{\gamma}(t) \triangleq x(t)$, results from applying $u_{\gamma}(s)$ to the model (2.8).

The solution (3.3)-(3.4) has been developed within the classical optimal control theory; however, not too much attention has been put on it. In fact, we suspect that great majority of the current robust control scientists are not aware of the existence of this solution. Probably, one of the main reasons that have lead to its oblivion is its unrealizable nature. Note that, in order to compute 
$u_{\gamma}(t)$, the equations (3.4a)-(3.4b) should be solved backwards time; starting from the terminal conditions $S(T)=S_{T}, v(T)=0$ and integrating up to the current time $t$. This implies that the disturbances should be known during all ${ }^{1}$ the future time interval in order to compute the current value of $u_{\gamma}$. Needless to say, this is not the case in practical applications -where, at least with the definition of the disturbance signals given in Sec. 2.1, the future disturbances are always unknown.

Albeit if it was assumed that $\omega$ is perfectly known, Eqs. (3.4a)-(3.4b) are not very "friendly" for a practical implementation. Specially due to (3.4a), which involves the integration of a matrix differential equation that necessarily needs to be performed by iterative numerical methods (Lewis et al. 2012).

In what follows, it is shown that this apparently complex controller can be analytically re-expressed in a highly simple form: $u_{\gamma}(t)=K_{x} x(t)+u_{f f}(\omega)$; being $u_{f f}(\omega)$ a functional depending on the future disturbance.

\section{Rewriting $u_{\gamma}(t)$ in an explicit -i.e. non-differential-form}

First of all, from all the signal $u_{\gamma}(s)$ in (3.3), we are only interested on its current value, $u_{\gamma}(t)$; as this is the one that is truly valid for control purposes. Note that, the rest of the control signal -i.e. $u^{*}(s), s>t$ - can be simply discarded because, at the next time instant, the problem will be re-evaluated again according to the new system state. Thus, taking (3.3) at $s=t$ gives:

$$
u_{\gamma}(t)=-(\gamma R)^{-1} B_{2}^{T}[S(t) x(t)+v(t)] .
$$

The variables $S(t)$ and $v(t)$ can be computed by integrating (3.4) from $T$ to $t$. To this end, note that in Eq. (3.4a) the terminal cost $S_{T}$ was intentionally chosen to be the solution to the ARE (3.2). This notably simplifies the treatment of Eq. (3.4a) and it has no influence on the cost (3.1) as $T \rightarrow \infty$. As the matrix $S_{T}$ makes the RHS of (3.4a) null, the integration of (3.4a) backwards in time, starting from $T$ up to the current time $t$, simply results in:

$$
\dot{S}(s)=0, \quad S(s)=S_{T}, \quad \text { for all } s \in[t, T] .
$$

This notably simplifies the management of Eq. (3.4) as, now, the RHS of the involved matrices -i.e. $\left[A-B_{2}(\gamma R)^{-1} B_{2}^{T} S(s)\right]^{T}$ and $S(s) B_{1}$ - become time-

\footnotetext{
${ }^{1}$ As it may be seen at the end of the following section, not all the future time instants should be considered but just a nearby future time interval.
} 
invariant. This equation can now be written as follows:

$$
-\dot{v}(s)=A_{c l}^{T} v(s)+S_{T} B_{1} \omega(s), \quad v(T)=0, s \in[t, T)
$$

being $A_{c l} \triangleq\left[A-B_{2}(\gamma R)^{-1} B_{2}^{T} S_{T}\right]$ a Hurwitz -i.e. stable- matrix ${ }^{2}$.

With the simplification of (3.4a)-(3.4b) into (3.6)-(3.7); the mathematical expression of $u_{\gamma}(t)$ can be straightforwardly obtained. To this purpose, it is just needed to integrate (3.7) from $T$ to $t$ and apply its terminal condition, giving:

$$
v(t)=\int_{t}^{T} e^{A_{c l}^{T}(s-t)} S_{T} B_{1} \omega(s) d s, \quad s \in[t, T]
$$

where it can be checked that $v(T)=0$-i.e. it satisfies the terminal conditionand, by Leibniz, it also satisfies the differential equation (3.7).

Substituting (3.6) and (3.8) into (3.3), and taking $T \rightarrow \infty$, the analytical expression of the controller $u_{\gamma}(t)$ is obtained as:

$$
u_{\gamma}(t)=K_{x} x(t)+K_{v} \int_{t}^{\infty} e^{A_{c l}^{T}(s-t)} S_{T} B_{1} \omega(s) d s
$$

where $K_{x} \triangleq-(\gamma R)^{-1} B_{2}^{T} S_{T} ; K_{v} \triangleq-(\gamma R)^{-1} B_{2}^{T} ; S_{T}$ is the solution to (3.2); and $A_{c l} \triangleq\left[A-B_{2}(\gamma R)^{-1} B_{2}^{T} S_{T}\right]$.

\section{Comments about $u_{\gamma}(t)$}

Note that -as discussed in Sec. 2.4- the controller $u_{\gamma}(t)$ contains the classical composite structure formed by state-feedback plus disturbance feed-forward. However, this composite structure has not been assumed beforehand and, moreover, the disturbance feed-forward term appears as a future-dependent integral and not as the commonly employed static-gain or stable filter multiplied by the actual disturbance; e.g. (Li et al. 2012; Khlebnikov 2016; Yang et al. 2011; Castillo et al. 2019b).

In the absence of disturbances, the integral is null and $u_{\gamma}(t)$ is reduced to the standard LQR. This property was also mentioned in Sec. 2.4.

Finally, for any bounded disturbance, the integral in (3.9) is finite as $e^{A_{c l}^{T}(s-t)}$, with $s \in[t, \infty)$, exponentially vanish to zero. Thus, in order to implement $u_{\gamma}(t)$,

\footnotetext{
${ }^{2}$ In fact $A_{c l}$ is the closed-loop matrix of the standard LQR regulator; which is proved to be Hurwitz (Lewis et al. 2012)
} 
the disturbance should be known just from the present, $\omega(t)$, to a near future, $\omega(t+h)$, where $h$ makes $e^{A_{c l}^{T} h}$ almost null.

Clearly, due to this property, the controller $u_{\gamma}(t)$ is unrealizable in practice as it depends on the future disturbance. However its mathematical expression (3.9) opens the door to new methodologies for synthesizing DOBCs that approximately implement it. How to do develop this approach is the main purpose of the following sections.

\subsection{Synthesizing DOBCs that approximately implement $u_{\gamma}(t)$}

The following theorem constitutes the main result of this chapter, as it shows how to synthesize DOBCs of the form $u(t)=K_{x} \hat{x}(t)+\sum_{i=0}^{r} K_{\omega, i} \hat{\omega}^{(i)}(t)$ that approximately implement $u_{\gamma}(t)$ :

Theorem 3.3.1. Under Assumptions 3.1-3.2, consider any estimations, $\hat{x}(t)$, $\hat{\omega}_{r}(t)$, of the variables $x(t)$ and $\omega_{r}(t) \triangleq\left[\omega^{T}(t), \dot{\omega}^{T}(t), \ldots, \omega^{(r) T}(t)\right]^{T}$ in $(2.8)$, respectively. Then, the optimal controller $u_{\gamma}(t)-(2.9)$, (3.9)-can be approximately implemented by:

$$
u(t)=K_{x} \hat{x}(t)+K_{v} \Phi \hat{\omega}_{r}(t)
$$

with an error equal to:

$$
u_{\gamma}(t)-u(t)=\mathcal{O}_{e}(t)+\mathcal{O}_{r}(t)
$$

being $K_{x} \triangleq-(\gamma R)^{-1} B_{2}^{T} S_{T} ; K_{v} \triangleq-(\gamma R)^{-1} B_{2}^{T} ;$ and

$$
\begin{aligned}
& \Phi \triangleq\left[\left(-A_{c l}^{T}\right)^{-1} S_{T} B_{1},\left(-A_{c l}^{T}\right)^{-2} S_{T} B_{1},[\ldots],\left(-A_{c l}^{T}\right)^{-(r+1)} S_{T} B_{1}\right], \\
& \mathcal{O}_{e}(t) \triangleq K_{x}[x(t)-\hat{x}(t)]+K_{v} \Phi(\tau)\left[\boldsymbol{\omega}_{r}(t)-\hat{\boldsymbol{\omega}}_{r}(t)\right], \\
& \mathcal{O}_{r}(t) \triangleq K_{v}\left(-A_{c l}^{T}\right)^{-(r+1)} \int_{t}^{\infty} e^{A_{c l}^{T}(s-t)} S_{T} B_{1} \omega^{(r+1)}(s) d s .
\end{aligned}
$$

with $A_{c l} \triangleq\left[A-B_{2}(\gamma R)^{-1} B_{2}^{T} S_{T}\right]$ and $S_{T}$ the solution to the ARE (3.2).

Proof. The proof follows by recursively integrating by parts the disturbance dependent term in (3.9). Let $\phi_{i} \triangleq \int_{t}^{\infty}\left(-A_{c l}^{T}\right)^{-i} e^{\left(-A_{c l}^{T}\right)(t-s)} S_{T} B_{1} \omega^{(i)}(s) d s$. Under Ass. 3.2, $\phi_{i}$ can be integrated by parts for any $i<r+1$ :

$$
\begin{aligned}
\phi_{i}=\left[-\left(-A_{c l}^{T}\right)^{-(i+1)} e^{\left(-A_{c l}^{T}\right)(t-s)} S_{T} B_{1} \omega^{(i)}(t)\right]_{s=t}^{s \rightarrow \infty} \\
\\
+\int_{t}^{\infty}\left(-A_{c l}^{T}\right)^{-(i+1)} e^{\left(-A_{c l}^{T}\right)(t-s)} S_{T} B_{1} \omega^{(i+1)}(s) d s .
\end{aligned}
$$


The integral term in the RHS (3.13) equals to $\phi_{i+1}$. In addition, as $A_{c l}$ is Hurwitz, the exponential $e^{\left(-A_{c l}^{T}\right)(t-s)}$ vanishes with $s \rightarrow \infty$. Therefore, (3.13) provides the following recursive equation:

$$
\phi_{i}=\left(-A_{c l}^{T}\right)^{-(i+1)} S_{T} B_{1} \omega^{(i)}(t)+\phi_{i+1} ;
$$

which straightforwardly enables to express $\phi_{0}$ as:

$$
\phi_{0}=\left(-A_{c l}^{T}\right)^{-1} S B_{1} \omega(t)+[\ldots]+\left(-A_{c l}^{T}\right)^{-(r+1)} S B_{1} \omega^{(r)}(t)+\phi_{r+1} .
$$

The theorem follows by substituting $\phi_{0}=\int_{t}^{\infty} e^{A_{c l}^{T}(s-t)} S_{T} B_{1} \omega(s) d s$ into (3.9); and denoting $\phi_{r+1} \triangleq \mathcal{O}_{r}$.

As mentioned in Sec. 2.2, the existing literature on DOBs has provided with a wide number of efficient algorithms and numerical methods for estimating -in real-time- the variables $x(t)$ and $\omega_{\boldsymbol{r}}(t)$, in $(3.10)$, through the historical set of sensor readings $y(t)$. Some examples are the ones by Luenberger 1964; Luenberger 1966; Schweppe 1968; Meditch et al. 1973; Bhattacharyya 1978; Darouach et al. 1994; Corless et al. 1998; Xiong et al. 2003; Chang 2006; Kim et al. 2010; Chakrabarty et al. 2017; Su et al. 2018. Any of these DOBs could be combined with Thm. 3.3.1 in order to produce a DOBC that approximates the optimal controller $u_{\gamma}(t)$.

\section{Resulting implementation errors}

The approximation of $u_{\gamma}(t)$ by Thm. 3.3.1 is subject to unavoidable errors -i.e. $\mathcal{O}_{e}(t)$ and $\mathcal{O}_{r}(t)$. These errors appear as a direct consequence of not having all the necessary information in order to directly compute the optimal control signal $u_{\gamma}(t)$. They deserve a bit further discussion as they mainly define the degree of sub-optimality of the DOBC approximation.

By Thm. 3.3.1, the implementation error is caused by two terms: i) $\mathcal{O}_{e}(t)$, which just depends on the current observation errors: $x(t)-\hat{x}(t), \boldsymbol{\omega}_{\boldsymbol{r}}(t)-\hat{\boldsymbol{\omega}}_{\boldsymbol{r}}(t)$; and ii) $\mathcal{O}_{r}(t)$, which, under perfect observation conditions (i.e. $\mathcal{O}_{e}(t)=0$ ), represents the error caused by computing $u_{\gamma}(t)$ just with the current disturbance state, $\boldsymbol{\omega}_{\boldsymbol{r}}(t)$, instead of computing it by (3.9) with the whole disturbance function: $\omega:[t, \infty) \rightarrow \mathbb{R}^{q}$. This error can be nicely seen as the unavoidable error appearing as a consequence of not knowing the future disturbance behavior.

On the one hand, the term $\mathcal{O}_{r}(t)$ can be bounded by:

$$
\left\|\mathcal{O}_{r}(t)\right\| \leq \mu\left\|K_{v}\left(-A_{c l}^{T}\right)^{-(r+1)}\right\| \epsilon_{r+1}
$$


being $\mu \triangleq \int_{t}^{\infty}\left\|e^{A_{c l}^{T}(s-t)} S_{T} B_{1}\right\| d s$.

Bound (3.16) shows that it is possible to make $\left\|\mathcal{O}_{r}(t)\right\|$ arbitrarily small through an appropriate choice of the parameters $\gamma$ and/or $r$. Reducing $\gamma$ makes the eigenvalues of $A_{c l}=A-B_{2}(\gamma R)^{-1} B_{2}^{T} S_{T}$ more negative (Di Ruscio 1992); This contributes to reduce the RHS of (3.16). On the other hand, -if $\omega$ is sufficiently differentiable ${ }^{3}$ - increasing $r$ contributes to exponentially decrease the size of $\mathcal{O}_{r}$.

Conversely, the term $\mathcal{O}_{e}$ entirely depends on how the observations are computed. It mainly depends on the capability of the DOB to give accurate estimates of $x(t)$ and $\boldsymbol{\omega}_{r}(t)$. Thus, accurate observations should be required in order to reduce this term.

\subsection{Application: design of a high-order ESO-based controller}

For the sake of completeness, let us show how a high-order Extended State Observer (ESO)-based feedback controller could be easily synthesized with this new methodology. The ESOs are, nowadays, one of the most popular algorithms to estimate in real-time the system and disturbance states due to their simplicity -e.g. (Castillo et al. 2018; Chen et al. 2016; Chang 2006; Castillo et al. 2020b). Let us show how they could be employed under this novel feedback design.

Consider the following high-order ESO-based controller:

$$
\begin{aligned}
\dot{\hat{\eta}}(t) & =\left[\bar{A}-L \bar{C}_{2}\right] \hat{\eta}(t)+\bar{B}_{2} u(t)+L y(t), \\
u(t) & =K \hat{\eta}(t),
\end{aligned}
$$

being $\hat{\eta} \in \mathbb{R}^{l}, l=n+(r+1) q$,

$$
\begin{aligned}
& \bar{A} \triangleq\left[\begin{array}{cc}
A & B_{1} \Pi \\
0 & \Upsilon
\end{array}\right], \quad \bar{B}_{2} \triangleq\left[\begin{array}{c}
B_{2} \\
0_{(r+1) q \times m}
\end{array}\right], \quad \bar{C}_{2} \triangleq\left[\begin{array}{ll}
C_{2} & 0_{p \times(r+1) q}
\end{array}\right], \\
& \Upsilon \triangleq\left[\begin{array}{cc}
0_{r q \times q} & I_{r q} \\
0_{q \times q} & 0_{q \times r q}
\end{array}\right], \quad \Pi \triangleq\left[\begin{array}{ll}
I_{q} & 0_{q \times r q}
\end{array}\right] \quad K \triangleq\left[\begin{array}{ll}
K_{x} & K_{v} \Phi
\end{array}\right],
\end{aligned}
$$

and $L \in \mathbb{R}^{l \times p}$ a free-design matrix.

\footnotetext{
${ }^{3}$ meaning that its high-order derivatives exist and that their associated upper bounds $\epsilon_{r+1}$ are sufficiently-small.
} 
Eq. (3.17a) is a conventional high-order ESO for the augmented system $\eta(t) \triangleq$ $\left[x^{T}(t), \boldsymbol{\omega}_{\boldsymbol{r}}^{T}(t)\right]^{T}$; whose dynamics - under Asm. 3.2 - is given by:

$$
\dot{\eta}(t)=\bar{A} \eta(t)+\bar{B}_{2} u(t)+\bar{B}_{1} \omega^{(r+1)}(t),
$$

with $\bar{B}_{1} \triangleq\left[0_{q \times(n+r q)}, I_{q}\right]^{T}$.

It thus provides with the needed estimates $\hat{x}(t), \hat{\omega}_{\boldsymbol{r}}(t)$ to implement Eq. (3.10) -which has been inserted in (3.17b).

By Thm. 3.3.1, Eq. (3.17) is approximately implementing $u_{\gamma}(t)$ with an error equal to (3.11); where $\mathcal{O}_{e}(t)$ is, in this case, given by:

$$
\begin{aligned}
\dot{e}_{\eta}(t) & =\left(\bar{A}-L \bar{C}_{2}\right) e_{\eta}(t)+\bar{B}_{1} \omega^{(r+1)}(t), \\
\mathcal{O}_{e}(t) & =K e_{\eta}(t),
\end{aligned}
$$

being $e_{\eta} \triangleq \eta(t)-\hat{\eta}(t)$.

The gain $L$ could be tuned through Eq. (3.19) in order to explicitly minimize $\mathcal{O}_{e}(t)$. This can be done by standard numerical methods, such as Lyapunovbased convex-optimization or $\mathcal{H}_{\infty}$ designs (Scherer et al. 2000). In what follows, let us introduce a LMI-design methodology that guarantees: i) an upper bound of the form: $\left\|\mathcal{O}_{e}(t)\right\| \leq \epsilon_{r+1} \sqrt{\frac{1}{2 \alpha \delta}}$, being $\alpha \delta$ a positive constant to be maximized; ii) that the eigenvalues of $\left(\bar{A}-L \bar{C}_{2}\right)$ belong to a given closed region of the complex plane.

\section{LMI-based design methodology}

Let us consider that:

Assumption 3.3. $\left(\bar{A}, \bar{C}_{2}\right)$ is observable.

The design procedure is summarized in the next lemma:

Lemma 3.4.1. Consider a closed subset $\mathcal{D}$ of the complex plane defined by $\mathcal{D} \triangleq\left\{z \in \mathbb{C} \mid N+z M+z^{*} M^{T} \prec 0\right\}$, where $N=N^{T}$ and $M$ are real matrices ${ }^{4}$ and $z^{*}$ denotes the conjugate of $z$. Under Asm. 3.3, let there exist positive constants $\alpha>0, \delta>0$, a symmetric and positive definite matrix $P \in \mathbb{R}^{l \times l}$ and

\footnotetext{
4 Some examples of subsets $\mathcal{D}$ are given in Chilali et al. 1996; Chilali et al. 1999.
} 
a matrix $Y \in \mathbb{R}^{l \times p}$ such that

$$
\begin{aligned}
& {\left[\begin{array}{cc}
P \bar{A}+\bar{A}^{T} P-Y \bar{C}_{2}-\bar{C}_{2}^{T} Y^{T}+2 \delta P & P \bar{B}_{1} \\
\bar{B}_{1}^{T} P & -I_{q}
\end{array}\right] \preceq 0} \\
& P-\alpha K^{T} K \succeq 0, \\
& N \otimes P+M \otimes\left(P \bar{A}-Y \bar{C}_{2}\right)+M^{T} \otimes\left(P \bar{A}-Y \bar{C}_{2}\right)^{T} \prec 0,
\end{aligned}
$$

Then, if $L=P^{-1} Y$, the error term $\mathcal{O}_{e}(t)$, in (3.19), exponentially approaches to the ball

$$
\left\|\mathcal{O}_{e}(t)\right\| \leq \epsilon_{r+1} \sqrt{\frac{1}{2 \alpha \delta}}
$$

for any $e_{\eta}(0)$ and, moreover, eig $\left(\bar{A}-L \bar{C}_{2}\right) \in \mathcal{D}$.

Proof. Refer to Castillo et al. 2020b.

Remark 3.1. In order to minimize $\mathcal{O}_{e}$, Lemma 3.4 .1 can be optimized to maximize the product $\alpha \delta$. This can be performed by standard LMI solvers as (3.20a)-(3.20c) becomes an LMI in all its variables if $\delta$ is set as a fixed parameter. An LMI solver can be employed to maximize $\alpha$, subject to (3.20a)$(3.20 \mathrm{c})$, for different fixed values of $\delta$ until finding the maximum $\alpha \delta$.

\section{Conclusion}

The main contents of this section summarizes as:

Theorem 3.4.1. Under Assumptions 3.1-3.3, consider a matrix $L \in \mathbb{R}^{l \times p}$ satisfying the conditions of Lemma 3.4.1. Then, the controller $u_{\gamma}(t)$-defined in (2.9) - can be approximately implemented by the high-order ESO-based controller (3.17) with an error equal to: $u_{\gamma}(t)-\hat{u}_{\gamma}(t)=\mathcal{O}_{e}(t)+\mathcal{O}_{r}(t)$; where $\mathcal{O}_{r}(t)$ satisfy the bound (3.16) and $\mathcal{O}_{e}(t)$ is ultimately bounded by (3.21); being both null if $\epsilon_{r+1}=0$. 


\begin{tabular}{|c|c|c|} 
Param. & Meaning & Value \\
\hline$m_{c}$ & Carriage mass & $1000 \mathrm{~kg}$ \\
$g$ & Gravity constant & $9.81 \mathrm{~m} / \mathrm{s}^{2}$ \\
$k_{f}$ & Force constant & $9810 \mathrm{~N} / \mathrm{T}^{2}$ \\
$k_{b}$ & Flux constant & $0.0015 \mathrm{~T} \mathrm{~m} / \mathrm{A}$ \\
$R_{c}$ & Coil's resistance & $10 \Omega$ \\
$L_{c}$ & Coil's inductance & $0.1 \mathrm{H}$ \\
$N_{c}$ & Number of turns & 2000 \\
$A_{p}$ & Pole face area & $0.01 \mathrm{~m}^{2}$ \\
$B_{0}$ & Nominal flux density & $1 \mathrm{~T}$ \\
$I_{0}$ & Nominal current & $10 \mathrm{~A}$ \\
$z_{t, 0}-z_{0}$ & Nominal air gap & $0.015 \mathrm{~m}$ \\
$V_{c, 0}$ & Nominal voltage & $100 \mathrm{~V}$ \\
\hline
\end{tabular}

Table 3.1: Operating conditions and parameters Michail 2009; Yang et al. 2011.

\subsection{Comparative example: magnetic levitation control}

Let us consider a MAGLEV system; whose identified dynamics is (Michail 2009; Yang et al. 2011):

$$
\begin{aligned}
& \frac{\mathrm{d}^{2} z}{\mathrm{dt}^{2}}=g-\frac{k_{f}}{m_{c}}\left(\frac{I}{z-z_{t}}\right)^{2}, \\
& V_{c}=R_{c} I+\frac{\mathrm{d} I}{\mathrm{dt}} L_{c}+N_{c} A_{p} \frac{\mathrm{d} B}{\mathrm{dt}}, \\
& B=k_{b} \frac{I}{z-z_{t}},
\end{aligned}
$$

where $z$ represents the electromagnet position, $z_{t}$ is the rail's position, $z-z_{t}$ stands for the air gap between the magnet and the rail, $I$ is the coil's current, $V_{c}$ is the coil's voltage and $B$ is the magnetic flux.

\subsubsection{Simulation scenario}

The simulation scenario is the same as the one reported in (Yang et al. 2011; Michail 2009). The system parameters, as well as the operating conditions, are summarized in Table 3.1.

The simulation emulates -as illustrated in Figs. 3.1-3.2- changes in the rail's position, $z_{t}(t)$. The first one -Fig. $3.1-$ is the same than the one reported (Yang 


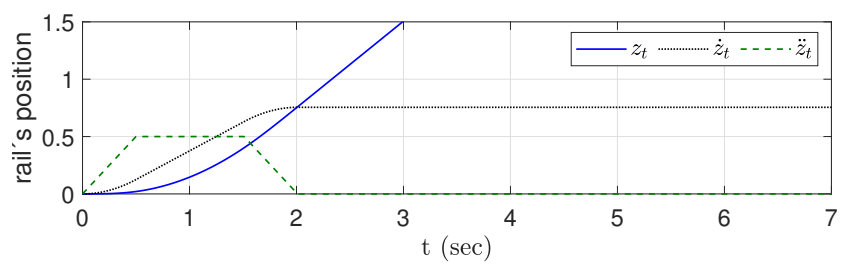

Figure 3.1: Change in the rail's slope (Michail 2009; Yang et al. 2011).

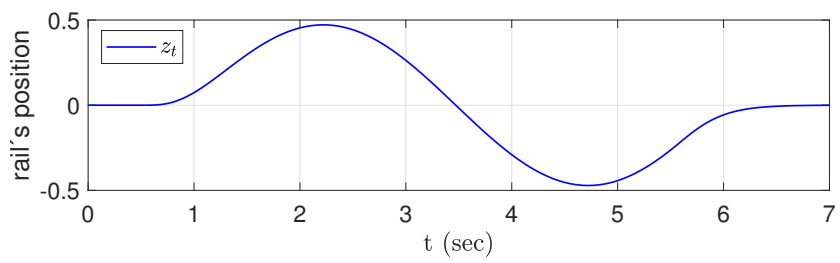

Figure 3.2: Sinusoidal variation of the rail's position (Frequency: $0.2 \mathrm{~Hz}$. Amplitude: 0.5 . Filter order: 3. Cut-off frequency $=1 \mathrm{~Hz}$ ).

et al. 2011); it represents a smooth change in the rail's slope. The second one -Fig. 3.2- represents a smooth sinusoidal variation of the rail's position. In both cases, the controller needs to automatically generate a voltage input $V_{c}(t)$ in order to maintain the nominal air-gap; i.e. $z(t)-z_{t}(t) \approx z_{t, 0}-z_{0}=0.015 \mathrm{~m}$.

The simulator solves Eq. (3.22), starting from steady-state conditions, and taking as inputs the rail position, $z_{t}(t)$, and the voltage, $V_{c}(t)$, which is generated by the controller. In order to generate this voltage, the controller has access to the variables $z-z_{t}, I$, and $\dot{z}$; which represent the measured variables of the MAGLEV system.

\subsubsection{DOBC design comparative}

A DOBC was proposed to this problem by Yang et al. 2011. It was based on the following LTI disturbed model:

$$
G \triangleq\left\{\begin{array}{l}
\dot{x}(t)=A x(t)+B_{2} u(t)+B_{1} \omega(t), \\
y(t)=C_{2} x(t), \\
z(t)=I_{3} x(t),
\end{array}\right.
$$

being $x \triangleq\left[\Delta I, \dot{z}, \Delta\left(z_{t}-z\right)\right]^{T}$, with $\Delta I=I-I_{0}$ and $\Delta\left(z_{t}-z\right)=\left(z_{t}-z\right)-$ $\left(z_{t, 0}-z_{0}\right)$ variations in $I$ and $z-z_{t}$ with respect to the operating point; $u=\Delta V_{c}=V_{c}-V_{c, 0}$ variations in the coil's voltage; $\omega(t) \in \mathbb{R}^{3}$ three unknown 
disturbance inputs and:

$$
\begin{aligned}
& A=\left[\begin{array}{ccc}
-\frac{R_{c}}{L_{c}+\frac{k_{b} N_{c} A_{p}}{G_{0}}} & -\frac{k_{b} N_{c} A_{p} I_{0}}{G_{0}^{2}\left(L_{c}+\frac{k_{b} N_{c} A_{p}}{G_{0}}\right)} & 0 \\
-\frac{2 k_{f} k_{b}^{2} I_{0}}{m_{c} G_{0}^{2}} & 0 & \frac{2 k_{f} k_{b}^{2} I_{0}^{2}}{m_{c} G_{0}^{3}} \\
0 & -1 & 0
\end{array}\right], B_{2}=\left[\begin{array}{c}
\frac{1}{L_{c}+\frac{k_{b} N_{c} A_{p}}{G_{0}}} \\
0 \\
0
\end{array}\right], B_{1}=I_{3} \\
& C_{2}=I_{3} .
\end{aligned}
$$

The nominal part of $(3.23)$-i.e. $\left(A, B_{2}, C_{2}\right)$ - corresponds to a linearized version of (3.22) around the operating point. The variable $y(t)=\left[\Delta I, \dot{z}, \Delta\left(z_{t}-z\right)\right]^{T}$ represents the measurements taken from the system. In this case, these measures come from the simulator solving (3.22) - but, in a real application, it will directly come from the sensors. The variable $z(t)$ corresponds to the controlled variable; i.e. the one that should be maintained close to zero by the controller. It has been chosen to be equal to $x(t)$-i.e. we want all the variations close to zero- however, it could be also set to $z(t)=[0,0,1] x(t)$ in order to explicitly say that we are only interested in maintaining $\Delta\left(z_{t}-z\right)$ close to zero. Finally, three disturbance signals have been introduced in order to account for the unknown changes of the rail's position, $z_{t}(t)$, as well as the linearization residuals ${ }^{5}$.

The DOBC design considered by Yang et al. 2011 and Li et al. 2012 is:

$$
u(t)=K_{x} \hat{x}(t)+\tilde{K}_{\omega} \hat{\omega}(t) ;
$$

with $K_{x}=[-60.58,591.18,40061]$, and

$$
\begin{aligned}
\tilde{K}_{\omega} & \triangleq-\left[\Theta\left(A+B_{2} K_{x}\right)^{-1} B_{2}\right]^{-1} \Theta\left(A+B_{2} K_{x}\right)^{-1} B_{1}, \\
& =[-2.1,35.97,742.16]
\end{aligned}
$$

being $\Theta \triangleq[0,0,1]$.

The gain $K_{x}$ corresponds to the resulting LQR gain (2.11) with weighting matrices $C_{1}=I_{3}, R=1$ and $\gamma=0.1$; whereas $\tilde{K}_{\omega}$ has been designed in order to reject constant disturbance signals in the state $z(t)=\Theta x(t)$, i.e. the air gap. Refer to Yang et al. 2011 or Li et al. 2012 for further details.

This DOBC feedback design is, nowadays, one of the most popular solutions. In fact, it has been employed, analyzed and mentioned by many different works

\footnotetext{
${ }^{5}$ Note that, following the words given in Sec. 2.1, the measurement equation in (3.23) is comparing two things that are originally different. On the one hand, $y(t)$ comes from the solution of the nonlinear system (3.22); whereas $C_{2} x(t)$ represents the solution of (3.23). The disturbances need to be included in order to account for their possible discrepancies.
} 

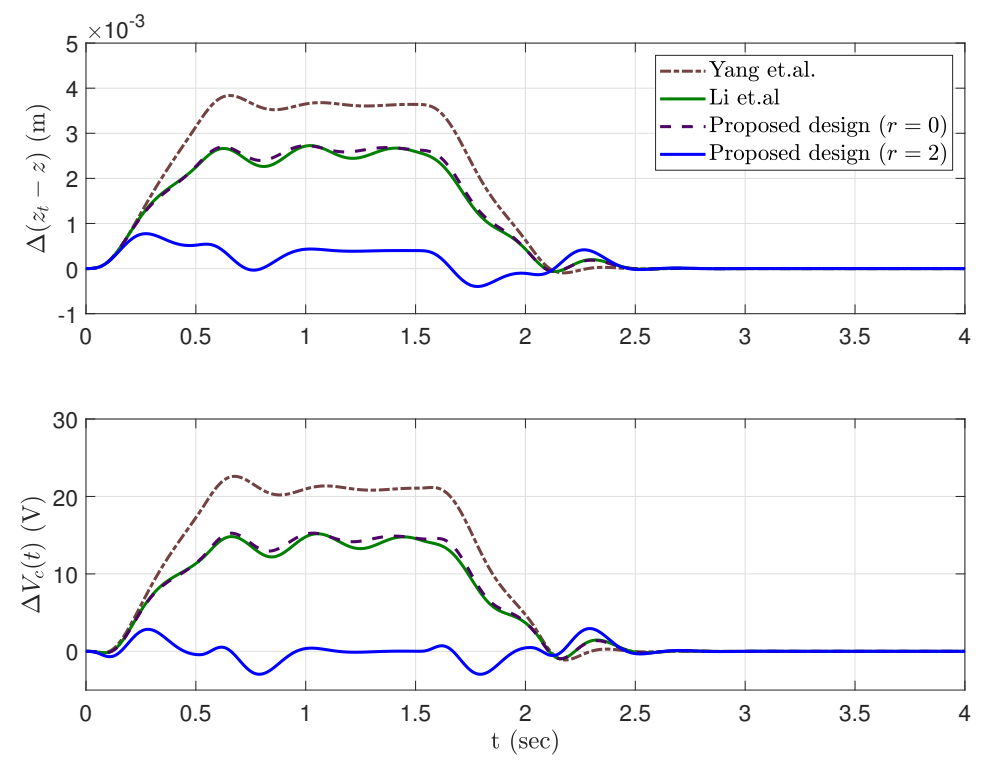

Figure 3.3: Simulation with respect to the change of the rail's slope.

- e.g. (Chen et al. 2016; Li et al. 2012; Castillo et al. 2019b; Khlebnikov 2016). Let us compare it with the feedback control derived in this chapter. If Thm. 3.3.1 is applied with the same values of $R$ and $\gamma$, it produces the following controllers:

$$
u(t)=K_{x} \hat{x}(t)+\sum_{i=0}^{r} K_{\omega, i} \hat{\omega}^{(i)}(t), \quad r \in \mathbb{N}
$$

where, now, $K_{\omega, 0}=[-0.098,30.63,-529.13], K_{\omega, 1}=[0.17,-0.4,-137.5]$, $K_{\omega, 2}=[0.007,-0.105,-4.08], \ldots$.

The integer $r$ is the parameter truncating the matrix $\Phi_{\infty}$ in Thm. 3.3.1. As shown in (3.16), increasing this parameter may contribute to reduce $\mathcal{O}_{r}(t)$.

\subsubsection{Simulation results}

Simulation results can be appreciated in Figs. 3.3-3.4; where it is seen that the DOBC design of this chapter is able to give better behavior in terms of performance and control effort. 

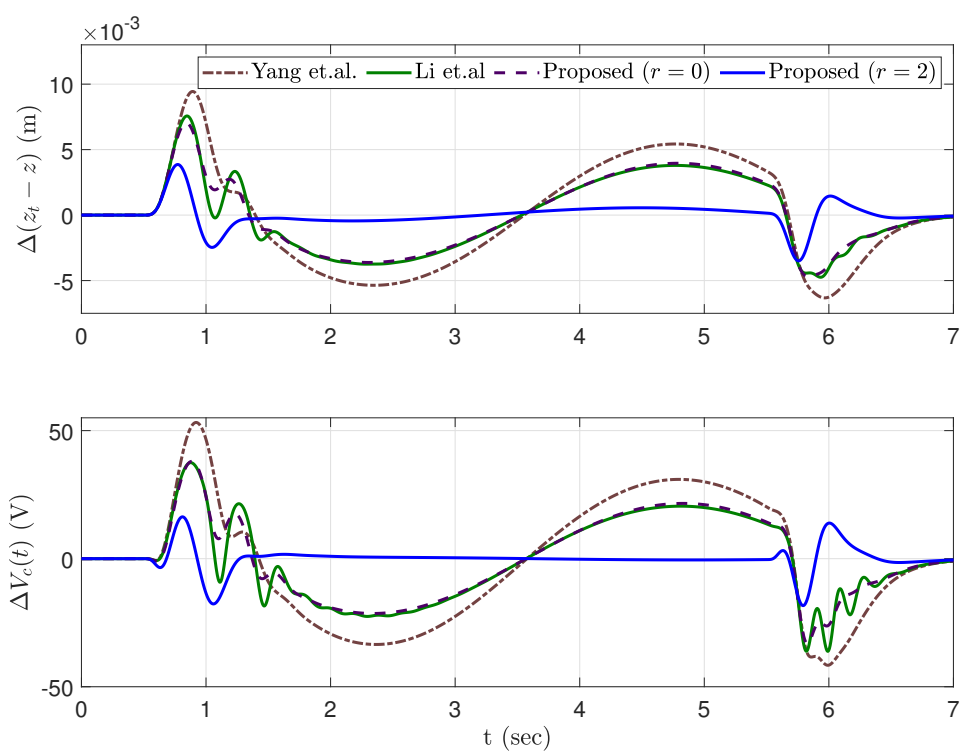

Figure 3.4: Simulation with respect to the sinusoidal variation of the rail's position.

In the simulations, the high-order ESO (3.17) has been employed in order to get the needed estimates $\hat{x}(t), \hat{\omega}(t), \hat{\dot{\omega}}(t), \hat{\ddot{\omega}}(t)$. The gain $L$ has been tuned to make $(\bar{A}-L \bar{C})$ Hurwitz, with poles approximately at $s=-20$; resulting in $L=[75.98,-634.9,9.207 \mathrm{e}-4 ;-1.96,80.75,1308 ; 0.001644,-0.9928,80.7$; $2464,-0.1201,0.05615 ; 0.1273,2464,-0.8936 ; 0.09981,0.5729,2461 ; 33670$, $-2.195,1.155 ; 2.875,33680,-15.43 ; 2.044,14.36,33620 ; 1.739 \mathrm{e}+05,-13.21$, $8.021 ; 21.61,1.74 \mathrm{e}+05,-86.04 ; 14.13,117,1.735 \mathrm{e}+05]$.

The green line corresponds to the controller (3.24); whereas the blue and purple lines correspond to the controller (3.25) with $r=0$ and $r=2$; respectively. As it can be seen, there is a significant jump in the controller performance from $r=0$ to $r=2$. The performance between (3.24) and (3.25), with $r=0$, is almost the same; however, the design criteria -and, consequently, the matrices $\tilde{K}_{\omega}$ and $K_{\omega, 0^{-}}$are still different. The brown line represents the same simulation as (Yang et al. 2011); whose major change is how the estimates are obtained. 


\begin{tabular}{|c|c|c|}
\hline Param. & Value & Quantifies \\
\hline$\tau_{1}$ & $49\left(\mathrm{~min}^{-1}\right)$ & Delay in absorption \\
$\tau_{2}$ & $47\left(\mathrm{~min}^{-1}\right)$ & Delay in absorption \\
$C_{l}$ & $2020(\mathrm{ml} / \mathrm{min})$ & Insulin clearance \\
$p_{2}$ & $1.6 \mathrm{e}-2\left(\mathrm{~min}^{-1}\right)$ & Delay in insulin action \\
$S_{I}$ & $8.11 \mathrm{e}-4(\mathrm{ml} / \mu \mathrm{U})$ & Insulin sensitivity \\
$k_{g e z i}$ & $2.20 \mathrm{e}-3\left(\mathrm{~min}^{-1}\right)$ & Effect of glucose to \\
& & reduce glucose itself \\
$k_{\text {egp }}$ & $1.33(\mathrm{mg} / \mathrm{dl} / \mathrm{min})$ & Glucose production rate \\
\hline
\end{tabular}

Table 3.2: Parameters in (3.26). Numerical values corresponding to the ones identified for Patient 1 in Kanderian et al. 2009.

\subsection{Comparative example: closed-loop glucose control}

Let us consider the following clinically identified glucose-insulin system for persons with type-1 diabetes (Stocker et al. 2006; Kanderian et al. 2009; Kanderian et al. 2012):

$$
\begin{aligned}
& \dot{v}_{1}(t)=-\frac{1}{\tau_{1}} v_{1}(t)+\frac{1}{\tau_{1} C_{l}} u_{I}(t), \\
& \dot{v}_{2}(t)=\frac{1}{\tau_{2}} v_{1}(t)-\frac{1}{\tau_{2}} v_{2}(t), \\
& \dot{v}_{3}(t)=p_{2} S_{I} v_{2}(t)-p_{2} v_{3}(t), \\
& \dot{v}_{4}(t)=-k_{g e z i} v_{4}(t)-v_{3}(t) v_{4}(t)+k_{e g p}+d(t), \\
& y\left(t_{k}\right)=v_{4}\left(t_{k}\right),
\end{aligned}
$$

where $u_{I}(t)$ represents the insulin delivery at Sub-Cutaneous (SC) level, $v_{1}(t)$ is the insulin concentration at $\mathrm{SC}$ level, $v_{2}(t)$ is the plasma insulin concentration, $v_{3}(t)$ is the insulin effect, $v_{4}(t)$ is the Glucose Concentration (GC) and $d(t)$ represents the glucose appearance following a meal.

\section{Simulation scenario}

System (3.26) is a clinically identified model for patients with type-1 diabetes. It represents -for a given person- how the GC in blood, $v_{4}(t)$, is affected by: i) insulin deliveries at sub-cutaneous level, i.e. $u_{I}(t)$; and ii) meals that the person takes, i.e. $d(t)$. All the parameters are defined in Table 3.2; corresponding to the ones identified for the Patient 1 by Kanderian et al. 2009. 


\begin{tabular}{|c|c|}
\hline Variable & Definition \\
\hline$v_{1}(t)$ & Insulin concentration at SC level \\
$v_{2}(t)$ & Plasma insulin concentration \\
$v_{3}(t)$ & Insulin effect \\
$v_{4}(t)$ & Glucose concentration \\
$u_{I}(t)$ & Insulin delivery at Sub-Cutaneous (SC) level \\
$d(t)$ & Glucose appearance following a meal \\
\hline
\end{tabular}

Table 3.3: Definition of variables in (3.26).

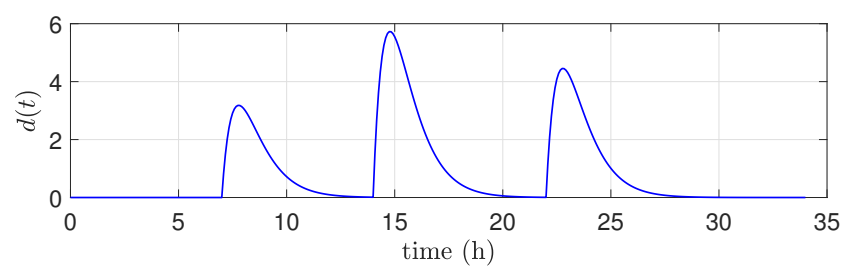

Figure 3.5: Disturbances caused by unannounced meals.

The simulation emulates a $24 \mathrm{~h}$-day where the patient eats $50 \mathrm{~g}$ of Carbohydrates (CHO) at $8 \mathrm{~h}, 90 \mathrm{~g} \mathrm{CHO}$ at $14 \mathrm{~h}$, and $70 \mathrm{~g} \mathrm{CHO}$ at $22 \mathrm{~h}$. These meals generate a glucose appearance as follows (Kanderian et al. 2009):

$$
d(t)=\frac{C_{H}(t)}{V_{G} \tau_{m}^{2}} t \mathrm{e}^{-\frac{t}{\tau_{m}}},
$$

where $C_{H}$ represents the amount of consumed carbohydrates, while $V_{G}=0.123$ and $\tau_{m}=47$ are the parameters identified for Patient 1 (Kanderian et al. 2009).

The waveform of $d(t)$ due to these meals is shown in Fig. 3.5.

The main objective is to design a controller that automatically injects insulin, $u_{I}(t)$, in response to unannounced/unknown meals that the person takes, $d(t)$. The GC should be kept at $v_{4}(0)=100(\mathrm{mg} / \mathrm{dl})$. In no case can be higher than $250(\mathrm{mg} / \mathrm{dl})$ or lower than $54(\mathrm{mg} / \mathrm{dl})$ as it may lead to severe hyperglycemia or hypoglycemia, respectively. After a meal, optimal values should range between $70(\mathrm{mg} / \mathrm{dl})<v_{4}(t)<180(\mathrm{mg} / \mathrm{dl})$.

The main limitations for the controller are: i) $u_{I}(t) \geq 0$, i.e. insulin can be injected but not subtracted; ii) $d(t)$ is unknown for the controller, i.e. the controller does not have information about when and how much the patient 
eats; iii) the controller only has access to discrete values of $v_{4}(t)$ with period $T=5 \mathrm{~min}$; which represents the measured variable.

The simulator solves Eq. (3.26), starting from steady state conditions -i.e. $v_{1}(0)=v_{2}(0)=13.68, v_{3}(0)=0.011, v_{4}(0)=100, u_{I}(0)=2.77 \mathrm{e} 4-$ and receiving as inputs $d(t)$ and the insulin deliveries $u_{I}(t)$, which are generated by the controller.

\subsubsection{DOBC design comparative}

A DOBC can be designed based on the following LTI disturbed model:

$$
G \triangleq\left\{\begin{array}{l}
\dot{x}(t)=A x(t)+B_{2} u(t)+B_{1} \omega(t), \\
y\left(t_{k}\right)=C_{2} x\left(t_{k}\right), \\
z(t)=C_{1} x(t),
\end{array}\right.
$$

where $x(t) \triangleq\left[v_{1}(t)-v_{1}(0), v_{2}(t)-v_{2}(0), v_{3}(t)-v_{3}(0), v_{4}(t)-v_{4}(0)\right]$ represents variations with respect to the steady-state conditions; $u(t)=u_{I}(t)-u_{I}(0)$; $\omega(t) \in \mathbb{R}$ an unknown disturbance signal; and

$A=\left[\begin{array}{cccc}-1 / \tau_{1} & 0 & 0 & 0 \\ 1 / \tau_{2} & -1 / \tau_{2} & 0 & 0 \\ 0 & p_{2} S_{I} & -p_{2} & 0 \\ 0 & 0 & -v_{4}(0) & -v_{3}(0)-k_{g e z i}\end{array}\right], B_{2}=\left[\begin{array}{c}1 /\left(\tau_{1} C_{l}\right) \\ 0 \\ 0 \\ 0\end{array}\right]^{T}, B_{1}=\left[\begin{array}{l}0 \\ 0 \\ 0 \\ 1\end{array}\right]^{T}$, $C_{2}=C_{1}=\left[\begin{array}{llll}0 & 0 & 0 & 1\end{array}\right]$.

The nominal part of $(3.28)$-i.e. $\left(A, B_{2}, C_{2}\right)$ - corresponds to a linearized version of (3.26) around the steady-state conditions. The variable $y\left(t_{k}\right)=$ $v_{4}\left(t_{k}\right)-v_{4}(0)$ represents the measurements taken from the system. In this case, these measures come from the simulator solving (3.22) - but, in a real application, it will directly come from glucose sensors. The variable $z(t)=y(t)$ corresponds to the controlled variable; i.e. the one that should be maintained close to zero by the controller. In this case, we want to reduce the variations of glucose with respect to the nominal value $v_{4}(0)=100$. Finally, one disturbance signal is introduced in order to account for the unknown meals, $d(t)$, as well as the linearization residuals ${ }^{6}$.

\footnotetext{
${ }^{6}$ As mentioned in footnote 5 . This is done in order to deal with the possible discrepancies between $y(t)$ and $C_{2} x(t)$.
} 
Similarly to Sec. 3.5; the DOBC feedback design considered by Yang et al. 2011 and Li et al. 2012 is:

$$
u(t)=K_{x} \hat{x}(t)+\tilde{K}_{\omega} \hat{\omega}(t)
$$

with $K_{x}=[-23.255,-22.434,-31920,3.0263]$, and

$$
\begin{aligned}
\tilde{K}_{\omega} & \triangleq-\left[C_{1}\left(A+B_{2} K_{x}\right)^{-1} B_{2}\right]^{-1} C_{1}\left(A+B_{2} K_{x}\right)^{-1} B_{1}, \\
& =25790 .
\end{aligned}
$$

The gain $K_{x}$ corresponds to the resulting LQR gain (2.11) with weighting matrices $R=1, \gamma=1 \mathrm{e}-4$; whereas $\tilde{K}_{\omega}$ is designed in order to reject constant disturbance signals in the controlled variable $z(t)$.

Similarly to Sec. 3.5, if Thm. 3.3.1 is applied with the same values of $R$ and $\gamma$, it produces the following controllers:

$$
u(t)=K_{x} \hat{x}(t)+\sum_{i=0}^{r} K_{\omega, i} \hat{\omega}^{(i)}(t), \quad r \in \mathbb{N}
$$

where, now, $K_{\omega, 0}=1945.3, K_{\omega, 1}=3.495 \mathrm{e} 5, K_{\omega, 2}=4.438 \mathrm{e} 7, \ldots$.

\section{Simulation results}

Simulation results can be appreciated in Fig. 3.6; where it is seen that the DOBC design of this chapter is able to give better behavior.

In the simulations, the high-order $\operatorname{ESO}$ (3.17) has been employed in order to get the needed estimates $\hat{x}(t), \hat{\omega}(t), \hat{\dot{\omega}}(t), \hat{\ddot{\omega}}(t)$. The gain $L$ has been optimized according to the procedure of Lemma 3.4.1; with the poles constrained in a closed circle around the origin with radius equal to 0.5 . The optimization procedure gives $L=[2.55 \mathrm{e}-9 ;-4.34 \mathrm{e}-9 ; 1.34 \mathrm{e}-9 ; 0.935 ; 0.497 ; 0.157 ; 0.0255]$.

The high-order ESO has been discretized by the first-order-hold method with period $T=5$ as there is only discrete measurements available. The computed control action is introduced to the system via a $\mathrm{ZOH}$.

In the simulations, the black line corresponds to the controller (3.29); whereas the blue and purple lines correspond to the controller (3.30) with $r=0$ and $r=2$; respectively. As it can be seen, there is a significant jump in the controller performance from $r=0$ to $r=2$. In fact, with $r=0$, the controller does almost nothing -note that the control action is almost null if compared 

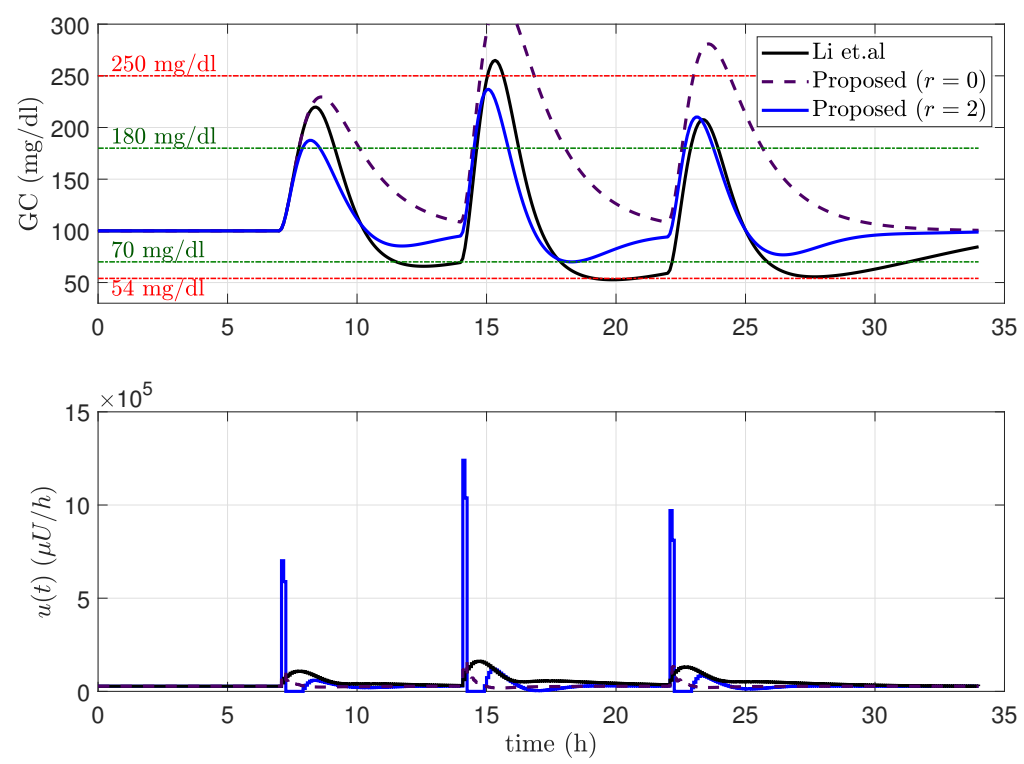

Figure 3.6: Comparative simulation results.

with the others. This is because, as it can be appreciated in Eq. (3.30), the matrices $K_{\omega, r}$ give more importance to the first and second derivatives of the disturbance signals instead of its absolute value. With $r=2$, the controller accurately generates a peak of insulin -i.e. an insulin bolus- at the same instant when a meal is detected. This is almost the same behavior that people with type- 1 diabetes normally do -i.e. they inject an insulin bolus to themselves before each meal.

Note that this behavior could not be achieved with the feedback (3.29), as it does not consider the disturbance derivatives.

\subsection{Conclusions}

In this chapter, DOBCs that approximately implement the optimal controller $u_{\gamma}(t)$ have been synthesized. To this end, the analytical formula for $u_{\gamma}(t)$ has been firstly developed and, then, it has been shown how to approximate it by DOBCs of the form $u(t)=K_{x} \hat{x}(t)+\sum_{i=0}^{r} K_{\omega, i} \hat{\omega}^{(i)}(t)$.

This novel DOBC feedback design is easily computable as it just requires to solve the standard Algebraic Ricatti Equation (ARE) and perform matrix mul- 
tiplications. Its major advantage is that it is indistinctly valid for MIMO systems with single/multiple matched/mismatched disturbances and, in addition to this, it offers an intuitive control tuning through the weighting parameters $R$ and $\gamma$. 



\section{Chapter 4}

\section{DOBC: Stability conditions}

In this chapter, some analyses about the resulting closed-loop formed by a DOBC and the system are given.

The main purpose is to make a clear distinction between two possible cases: i) the disturbance signals are not coupled with the nominal system-state; ii) the disturbance signals are coupled with the system-state. This distinction is crucial as it has different implications on the closed-loop performance and, even, on its stability. And both type of disturbances regularly appear in practice.

The technical contents of this chapter has been mainly extracted from (Castillo et al. 2019b; Castillo et al. 2017). 


\subsection{Introduction}

In Chapter 2, the disturbance signals were defined as some unknown inputs that are included in the model in order to guarantee that its output follows the information given by the sensors. It was mentioned that these signals can be estimated by DOB algorithms and, in Chapter 3, it was shown how to make use of these estimates in order to design controllers of the form $u(t)=$ $K_{x} \hat{x}(t)+\sum_{i=0}^{r} K_{\omega, i} \hat{\omega}^{(i)}(t), r \in \mathbb{N}$, that approximately implement the optimal control signal $u_{\gamma}$ - i.e. Eq. (2.9).

In this chapter, the attention is focused on the origin of those disturbance signals. In fact, two cases should be distinguished as they have different implications on the closed-loop performance and, even, on its stability. The question is: are the disturbance signals coupled with the system-state or not?

If the disturbances are coupled with the system, it means that a change in the system-state -e.g. changes of movement, velocity, position, temperature, pressure, flow-direction, etc- will also cause significant changes on the observed disturbance signals. On the other hand, non-coupled disturbances are signals that are mostly independent of the system-state.

Disturbances that mostly arise from modeling errors are coupled disturbances; whereas disturbances that mostly arise from interconnections with other independent systems are non-coupled disturbances. For example, in a drone flying at hover, the wind gusts are clearly non-coupled disturbances as they are mostly independent of the drone position. However, in a submarine diving in calm waters, the friction with the water is a coupled disturbance as it is mainly dependent on the submarine velocity. In the first case, the disturbances arise from the interconnection with other independent system; say, the environmental weather. In the second case, they mostly arise from modeling errors as, in fact, the friction of the submarine with the static water forms part of the submarine-system ${ }^{1}$.

In practice, disturbance signals would be a mixture of both - sometimes even hard to be distinguished. In fact, many authors -e.g. Han 2009; Wu et al. 2020; Huang et al. 2014; Xie et al. 2000- state that there is no point in establishing such distinction as, in the end, the disturbances are caused by something whose origin is uncertain and, from a control design perspective, they could be just regarded as some unknown time-varying functions.

\footnotetext{
${ }^{1}$ However, quantifying this effect could be excessively complex and, for simplicity, this friction is just "translated" to the disturbance signals.
} 
Nevertheless, from a control analysis point of view -i.e. making statements about how the controller will behave when applied to the system-, the distinction is crucial. Note that a controller that depends on the disturbance signals is being applied to the system and this controller modifies the system behavior and the system-state; therefore, if the disturbances are coupled with the system, the controller will be also modifying the disturbance signals to which it is dependent. There exist a clear feedback-loop here that, in some cases, could have very negative effects in the system performance ${ }^{2}$.

This section aims to analyze this closed-loop in order to establish the following result: the closed-loop system will always be stable in scenarios with coupled disturbances if the coupling is sufficiently weak. For non-coupled disturbances, the resulting closed-loop is always stable. Thus, analyzing this feedback provides the control-designer with some qualitative clues, say: i) although this robust control approach is able to deal with inner modeling errors ${ }^{3}$, it is important -in terms of stability and performance- that the nominal model contains the most relevant information about the system behavior. ii) the DOB bandwidth should be notably faster than the closed-loop system dynamics. In this way, -although there exist coupling between both the system-state and the disturbances- the DOBC is able to detect variations in the disturbances generated by the controlled system itself.

\section{Problem formulation and main assumptions}

Fig. 4.1 contains a block-diagram of the problem considered in this chapter. It is basically the same than the one of Fig. 2.2, but with the particularity that the disturbance input is now dependent on the system-state, $x$, and other non-coupled disturbances, $\tilde{\omega}$.

System $G$ is therefore rewritten as:

$$
G \triangleq\left\{\begin{array}{l}
\dot{x}(t)=A x(t)+B_{2} u(t)+B_{1} \omega(x, \tilde{\omega}(t)) \\
y(t)=C_{2} x(t) \\
z(t)=C_{1} x(t)
\end{array}\right.
$$

\footnotetext{
${ }^{2}$ In fact, these effects where experimentally observed in Castillo et al. 2019a; where the transient response of the drone rotors needed to be properly modeled and included as a part of the nominal model for the DOB. Neglecting this part of the model -or better said, accommodating this part of the model through the disturbance signals- had negative effects in closed-loop for high-observer bandwidths due to the coupling between the drone movements and the observed disturbances.

${ }^{3}$ In fact, in many cases, it is able to deal with unbelievable large modeling errors — refer, for example, to the ADRC literature (Huang et al. 2014; Han 2009; Gao 2006).
} 


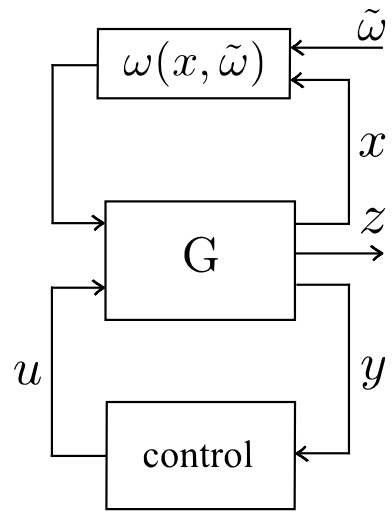

Figure 4.1: Four-block control problem with coupled disturbances.

Note that, in this model, the control inputs, $u(t)$, affect the system-state, $x(t)$, which also affects the disturbance input. This defines the closed-loop above mentioned, which is illustrated in Fig. 4.1.

The following assumption on the disturbance signal is considered for the subsequent analyses:

Assumption 4.1. There exist scalars, $\beta_{\omega} \geq 0, \beta_{\dot{\tilde{\omega}}} \geq 0, \beta_{d x} \geq 0, \beta_{d \tilde{\omega}} \geq 0$, such that,

$$
\begin{array}{ll}
\|\omega(x, \tilde{\omega}(t))\| \leq \beta_{\omega}, & \|\dot{\tilde{\omega}}(t)\| \leq \beta_{\dot{\tilde{\omega}}}, \\
\left\|\frac{\partial \omega}{\partial x}(x, \tilde{\omega}(t))\right\| \leq \beta_{d x}, \quad\left\|\frac{\partial \omega}{\partial \tilde{\omega}}(x, \tilde{\omega}(t))\right\| \leq \beta_{d \tilde{\omega}},
\end{array}
$$

for all $x \in \mathcal{B}_{r}, \mathcal{B}_{r} \triangleq\left\{x \in \mathbb{R}^{n} \mid\|x\| \leq \rho\right\}, \rho>0, t \geq t_{0}$.

Assumption 4.1 states that $\omega(x, \tilde{\omega}(t))$ and its partial derivatives are bounded in a closed set, $\mathcal{B}_{r}$, around the origin — not necessarily globally bounded. This basically states that, nearby an operating condition, the disturbance has a continuous dependency on $x$ - i.e. continuous variations in the nominalstate, $x$, generate continuous variations in $\omega$. This is what normally happens in practice.

The most important parameter in Asm. 4.1 is $\beta_{d x}$; which represents the coupling between $\omega$ and $x$. Big $\beta_{d x}$ implies that small variations in the system-state could cause big variations in the disturbance signal. As it may be seen, the closed-loop stability is strongly dependent on this parameter. Instability may appear for big $\beta_{d x}$. 
Thusly, what we look for is to find conditions for which any closed-loop system trajectory starting inside a set -say $x\left(t_{0}\right) \in \mathcal{E}_{0} \subseteq \mathcal{B}_{r}$ - will remain inside $\mathcal{B}_{r}$ for all $t>t_{0}$. This implies stability. In order to analyze this problem, it is considered that the control action is generated by

$$
u(t)=K_{x} \hat{x}(t)+K_{\omega, 0} \hat{\omega}(t) ;
$$

which corresponds to the one defined in Thm. 3.3.1 with $r=0$-or with the control laws employed in other works, such as: Li et al. 2012; Huang et al. 2014; Khlebnikov 2016; She et al. 2008. Also, it is considered that the estimates are given by the ESO (3.17a) with $r=0$. Similar results may hold for $r>0$ or for other type of observers.

\section{Notation}

The maximum and minimum eigenvalues of a given matrix, $P$, are denoted by $\bar{\lambda}(P)$ and $\underline{\lambda}(P)$, respectively.

Let $\xi \triangleq\left[x^{T}, e_{o}^{T}\right]^{T} \in \mathbb{R}^{2 n+q}$, with $x \in \mathbb{R}^{n}$ and $e_{o} \in \mathbb{R}^{n+q}$. A symmetric matrix $0<P_{i} \in \mathbb{R}^{(2 n+q) \times(2 n+q)}$, defines an ellipsoid in $\mathbb{R}^{2 n+q}$ given by

$$
\mathcal{E}_{i} \triangleq\left\{\xi \in \mathbb{R}^{2 n+q} \mid \xi^{T} P_{i} \xi \leq k_{i}, k_{i}>0\right\},
$$

whose projection onto $\mathbb{R}^{n}, \mathcal{E}_{i}^{\perp}$, is automatically defined by $P_{i}^{\perp} \in \mathbb{R}^{n}$ :

$$
\mathcal{E}_{i}^{\perp} \triangleq\left\{x \in \mathbb{R}^{n} \mid x^{T} P_{i}^{\perp} x \leq k_{i}, k_{i}>0\right\} .
$$

\subsection{Closed-loop stability conditions}

Let us define the observation error as

$$
e_{\eta} \triangleq\left[\begin{array}{l}
e_{\eta, x} \\
e_{\eta, \omega}
\end{array}\right] \triangleq\left[\begin{array}{c}
x-\hat{x} \\
\omega(x, \tilde{\omega}(t))-\hat{\omega}
\end{array}\right]=\eta-\hat{\eta} ;
$$

which, for the observer (3.17a) with $r=0$, it was proved to satisfy the following differential equation -i.e. (3.19):

$$
\dot{e}_{\eta}=(\bar{A}-L \bar{C}) e_{\eta}+\bar{B}_{f} \dot{\omega}(x, \tilde{\omega}(t)) ;
$$

where, now, $\dot{\omega}(x, \tilde{\omega}(t))=\frac{\partial \omega}{\partial x} \dot{x}+\frac{\partial \omega}{\partial \tilde{\omega}} \dot{\tilde{\omega}}$.

The control action in (4.2) can be rewritten as:

$$
\begin{aligned}
u=K_{x} \hat{x}+K_{\omega, 0} \hat{\omega} & =K_{x} x+K_{\omega, 0} \omega(x, \tilde{\omega}(t))-K_{x} e_{\eta, x}-K_{\omega, 0} e_{\eta, \omega} \\
& =K_{x} x+K_{\omega, 0} \omega(x, \tilde{\omega}(t))+E_{\eta} e_{\eta}
\end{aligned}
$$


where $E_{\eta} \triangleq-\left[K_{x}, K_{\omega, 0}\right]$.

Finally, by substituting (4.5) into (4.1) and incorporating (4.4), the following closed-loop is obtained:

$$
\dot{\xi}=\Phi_{c} \xi+\Gamma_{1} \omega(x, \tilde{\omega}(t))+\Gamma_{2} \dot{\omega}(x, \tilde{\omega}(t)),
$$

where $\xi \triangleq\left[x^{T}, e_{\eta}^{T}\right]^{T}$ and

$$
\Phi_{c} \triangleq\left[\begin{array}{cc}
A+B_{2} K_{x} & B_{2} E_{\eta} \\
0 & \bar{A}-L \bar{C}
\end{array}\right], \quad \Gamma_{1} \triangleq\left[\begin{array}{c}
B_{2} K_{\omega, 0}+B_{1} \\
0
\end{array}\right], \quad \Gamma_{2} \triangleq\left[\begin{array}{c}
0 \\
\bar{B}_{f}
\end{array}\right] .
$$

In the next sections, the stability of the closed-loop (4.6) is analyzed. The main purpose is to find conditions for which any system trajectory starting inside a set -say $x\left(t_{0}\right) \in \mathcal{E}_{0} \subseteq \mathcal{B}_{r}$ - will remain inside $\mathcal{B}_{r}$ for all $t>t_{0}$.

\section{Stability conditions}

Let us recall the next well-known result over which the subsequent stability conditions are developed:

Lemma 4.2.1. (ISS). Define $V(\xi(t))=\xi(t)^{T} P \xi(t)$, with $P>0$. Let $\bar{V}(t) \triangleq$ $V(\xi(t))$ be absolutely continuous and let $g_{1}(x(t), t), g_{2}(x(t), t)$ be essentially bounded functions, i.e. $\left\|g_{1}(x(t), t)\right\| \leq \alpha_{1},\left\|g_{2}(x(t), t)\right\| \leq \alpha_{2}$, for all $t \geq t_{0}$, with $\alpha_{1} \geq 0, \alpha_{2} \geq 0$. If there exist $\delta>0, \gamma_{1} \geq 0, \gamma_{2} \geq 0$ such that

$$
\dot{\bar{V}}(t)+\delta \bar{V}(t)-\gamma_{1}\left\|g_{1}(x(t), t)\right\|^{2}-\gamma_{2}\left\|g_{2}(x(t), t)\right\|^{2} \leq 0, \forall t \geq t_{0}
$$

then, the ellipsoid $\mathcal{E} \triangleq\left\{\xi \in \mathbb{R}^{2 n+q} \mid \xi^{T} P \xi \leq \frac{\gamma_{1} \alpha_{1}^{2}+\gamma_{2} \alpha_{2}^{2}}{\delta}\right\}$, is a positively invariant and exponentially attractive set, with decay rate $\delta / 2$, for $\xi(t)$.

Proof. The proof is similar to the one presented in Lemma 4.1 of Fridman 2014, where the term $b\|\tilde{\omega}(t)\|^{2}$ is substituted by $\gamma_{1}\left\|g_{1}(x(t), t)\right\|^{2}+\gamma_{2}\left\|g_{2}(x(t), t)\right\|^{2}$.

Lemma 4.2.1 states that, if we are able to find one Lyapunov function, $V(\xi(t))=$ $\xi(t)^{T} P \xi(t)$, such that (4.7) holds along the trajectories of (4.6); then the ellipsoidal set $\mathcal{E}$ satisfies: i) any trajectory of (4.6) starting inside it, remains inside forever; ii) any trajectory starting outside it, exponentially approaches to the interior of the ellipsoid -and then remains inside.

The above lemma is employed to establish the following result; which contains conditions to: i) guarantee that the trajectories of the closed-loop (4.6) remain 


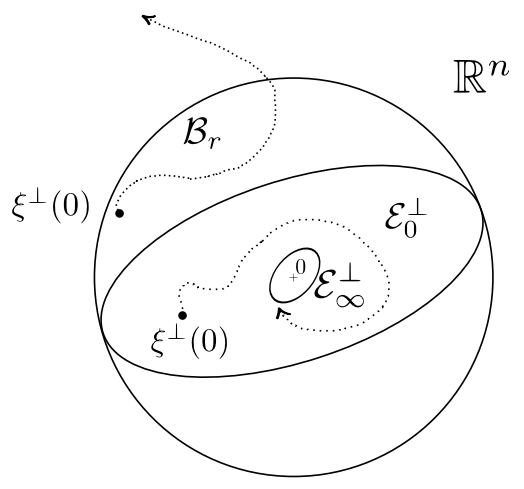

Figure 4.2: Illustration of the sets being considered in this problem.

inside the ball $\mathcal{B}_{r}$; ii) find the largest ellipsoidal set of allowable initial states; iii) find the smallest ellipsoidal set to which the trajectories converge.

Theorem 4.2.1. Let $i \triangleq\{0, \infty\}$. Under Asm. 4.1, let there exist positive definite $P_{i} \in \mathbb{R}^{(2 n+q) \times(2 n+q)}$ and scalars $\delta_{i}>0, \tau_{i}>0, \gamma_{1_{i}} \geq 0, \gamma_{2_{i}} \geq 0$, that satisfy the following LMIs:

$$
\Psi_{i s s}^{i} \triangleq\left[\begin{array}{cccc}
\psi_{i s s}^{i} & P_{i} \Gamma_{1}+\tau_{i} \beta_{d x}^{2} \Delta_{\xi}^{T} \Delta_{f} & P_{i} \Gamma_{2} & P_{i} \Gamma_{2} \\
(*) & -\gamma_{1_{i}}+\tau_{i} \beta_{d x}^{2} \Delta_{f}^{T} \Delta_{f} & 0 & 0 \\
(*) & (*) & -\tau_{i} & 0 \\
(*) & (*) & (*) & -\gamma_{2_{i}}
\end{array}\right] \leq 0
$$

with $\psi_{i s s}^{i} \triangleq P_{i} \Phi_{c}+\Phi_{c}^{T} P_{i}+\delta_{i} P_{i}+\tau_{i} \beta_{d x}^{2} \Delta_{\xi}^{T} \Delta_{\xi} ; \Delta_{\xi} \triangleq\left[\left(A+B_{2} K_{x}\right), B_{2} E_{\eta}\right]$ and $\Delta_{\omega} \triangleq\left(B_{2} K_{\omega, 0}+B_{1}\right)$; and the following inequalities:

$$
\sqrt{\frac{\gamma_{1_{i}} \beta_{f}^{2}+\gamma_{2_{i}}\left(\beta_{d \tilde{\omega}} \beta_{\dot{\tilde{\omega}}}\right)^{2}}{\underline{\lambda}\left(P_{i}^{\perp}\right) \delta_{i}}}<\rho .
$$

Then, for any trajectory, $\xi$, starting from the set:

$$
\mathcal{E}_{0} \triangleq\left\{\xi \in \mathbb{R}^{2 n+q} \mid \xi^{T} P_{0} \xi \leq \frac{\gamma_{1_{0}} \beta_{f}^{2}+\gamma_{2_{0}}\left(\beta_{d \tilde{\omega}} \beta_{\dot{\tilde{\omega}}}\right)^{2}}{\delta_{0}}\right\} ;
$$

the state-trajectory, $x$, of the closed-loop (4.6) does not leave the ball $\mathcal{B}_{r}$ and it exponentially approaches, with a decay rate $\delta_{\infty} / 2$, to the attractive ellipsoid

$$
\mathcal{E}_{\infty}^{\perp} \triangleq\left\{x \in \mathbb{R}^{n} \mid x^{T} P_{\infty}^{\perp} x \leq \frac{\gamma_{1_{\infty}} \beta_{f}^{2}+\gamma_{2_{\infty}}\left(\beta_{d \tilde{\omega}} \beta_{\dot{\tilde{\omega}}}\right)^{2}}{\delta_{\infty}}\right\} .
$$


Proof. Refer to Annex 1.

Figure 4.2 represents an illustration of the sets that are being considered in this problem. $\mathcal{B}_{r}$ is the ball where Assumption 4.1 has been established and, therefore, it is where the trajectories of the closed-loop system are required to belong. Theorem 4.2.1 states that, if it is possible to find two sets of parameters -i.e. $\left\{P_{0}, \delta_{0}, \tau_{0}, \gamma_{1_{0}}, \gamma_{2_{0}}\right\}$ and $\left\{P_{\infty}, \delta_{\infty}, \tau_{\infty}, \gamma_{1_{\infty}}, \gamma_{2_{\infty}}\right\}$ - such that (4.8)-(4.9) hold, then for any initial state $\xi\left(t_{0}\right) \triangleq\left[x^{T}\left(t_{0}\right), e_{\eta}^{T}\left(t_{0}\right)\right]^{T} \in \mathcal{E}_{0}$, the trajectory of $x(t)$ will not leave $\mathcal{B}_{r}$ and, moreover, it approaches to the attractive ellipsoid $\mathcal{E}_{\infty}^{\perp}$.

This was the required result, but still there is one main issue to be tackled: is it possible to find a set of parameters $\left\{P_{i}, \delta_{i}, \tau_{i}, \gamma_{1_{i}}, \gamma_{2_{i}}\right\}$ such that (4.8)-(4.9) hold? There are two options here. The first one is to employ a computer in order to look for those parameters and check the conditions. This can be done by standard LMI solvers. The second one is to prove that both conditions will always be satisfied provided that some parameters in Asm. 4.1 are sufficiently small or sufficiently big.

Both approaches are addressed in the following lines. In Sec. 4.3, it is shown how the LMIs (4.8) can be numerically optimized in order to get the specific values of $\left\{P_{0}, \delta_{0}, \tau_{0}, \gamma_{1_{0}}, \gamma_{2_{0}}\right\}$ and $\left\{P_{\infty}, \delta_{\infty}, \tau_{\infty}, \gamma_{1_{\infty}}, \gamma_{2_{\infty}}\right\}$ maximizing $\mathcal{E}_{0}$ and minimizing $\mathcal{E}_{\infty}$; respectively. Optimizing these parameters may be useful to make quantitative predictions of how the possible closed-loop trajectories will behave - provided that the numerical values of the bounds in Asm. 4.1 are known. This will be illustrated in Section 4.4.

Corollary 4.1 follows the second approach: it states that the closed-loop system will be stable for small-enough $\beta_{d x}$.

Corollary 4.1. (Stability for weakly-coupled disturbances). Consider that Assumption 4.1 is satisfied with a small-enough $\beta_{d x}$ and long-enough $\rho$. Then, if $\left(A+B_{2} K_{x}\right)$ and $(\bar{A}-L \bar{C})$ are Hurwitz, the trajectory, $x(t)$, of the closedloop system (4.6) will lie inside $\mathcal{B}_{r}$ for any initial-state $\xi\left(t_{0}\right) \triangleq\left[x^{T}\left(t_{0}\right), e_{\eta}^{T}\left(t_{0}\right)\right]^{T}$ sufficiently close to the origin.

Proof. Since $\left(A+B_{2} K_{x}\right)$ and $(\bar{A}-L \bar{C})$ are Hurwitz, there exist $P_{i}$ such that $P_{i} \Phi_{c}+\Phi_{c}^{T} P_{i}+\delta_{i} P_{i}<0$ for any given $\delta_{i}$. Then, by Schur complements, the LMI (4.8) -with $\gamma_{1_{i}} \geq 0, \gamma_{2_{i}} \geq 0, \tau_{i}>0$ - holds for small-enough $\beta_{d x}$. On the other hand, condition (4.9) is always satisfied if $\rho$ is big-enough. 
As a particular case, if the disturbances are not coupled with the system -i.e. they do not depend on $x$ or, directly, $\beta_{d x}=0$ - then, the closed-loop system will always be stable provided that $\left(A+B_{2} K_{x}\right)$ and $(\bar{A}-L \bar{C})$ are Hurwitz.

\subsection{Numerical optimization of the stability conditions}

In this section, it is shown how the sets of parameters $\left\{P_{0}, \delta_{0}, \tau_{0}, \gamma_{1_{0}}, \gamma_{2_{0}}\right\}$ and $\left\{P_{\infty}, \delta_{\infty}, \tau_{\infty}, \gamma_{1_{\infty}}, \gamma_{2_{\infty}}\right\}$ can be numerically optimized in order to find the biggest initial-state ellipsoid $\mathcal{E}_{0}$ and the smallest attractive ellipsoid $\mathcal{E}_{\infty}$.

The terminal ellipsoid $\mathcal{E}_{\infty}$ can be minimized by solving the following problem:

$$
\begin{aligned}
\max _{\left\{s_{\infty}, \alpha\right\}} & \alpha \\
\text { s.t. } & \Psi_{i s s}^{\infty} \leq 0, P_{\infty}>\alpha I_{2 n+q}, \\
& \gamma_{1_{\infty}} \beta_{f}^{2}+\gamma_{2_{\infty}}\left(\beta_{d \tilde{\omega}} \beta_{\dot{\tilde{\omega}}}\right)^{2}=\delta_{\infty}, \\
& \alpha>0, \delta_{\infty}>0, \tau_{\infty}>0, \gamma_{1_{\infty}} \geq 0, \gamma_{2_{\infty}} \geq 0 ;
\end{aligned}
$$

where the first constrain assures that condition (4.8) of Theorem 4.2.1 is satisfied. The second constraint assures that $\underline{\lambda}\left(P_{\infty}\right) \geq \alpha$. The third constraint forces the LHS of (4.9) to take the form $r_{\infty}=\sqrt{\frac{1}{\underline{\underline{\lambda}\left(P_{\infty}^{\perp}\right)}}} \leq \sqrt{\frac{1}{\alpha}}$. So, if $\alpha$ is maximized, then the sphere containing $\mathcal{E}_{\infty}$ is being minimized -and consequently the size of $\mathcal{E}_{\infty}$ is being reduced.

Maximizing $\mathcal{E}_{0}$ can be done by minimizing the condition number of $P_{0}$ (so that $\mathcal{E}_{0}$ is as similar as possible to a sphere), while forcing condition (4.9) to be strictly satisfied; i.e. as an equality. In this way, the largest ellipsoid such that its projection strictly fits inside $\mathcal{B}_{r}$ is obtained. This can be performed by solving the next optimization problem for different fixed (and decreasing) values of $\alpha$, until (4.9) holds as an equality:

$$
\begin{aligned}
\min _{\left\{s_{0}, \alpha, \gamma\right\}} & \gamma, \\
\text { s.t. } & \Psi_{i s s}^{0} \leq 0, P_{0}=\alpha P, \gamma I_{2 n+q}>P>I_{2 n+q}, \\
& \gamma_{1_{0}} \beta_{f}^{2}+\gamma_{2_{0}}\left(\beta_{d \tilde{\omega}} \beta_{\dot{\tilde{\omega}}}\right)^{2}=\delta_{0}, \\
& \gamma \geq 1, \alpha>0, \delta_{0}>0, \tau_{0}>0, \gamma_{1_{0}} \geq 0, \gamma_{2_{0}} \geq 0 ;
\end{aligned}
$$

where, the second and third constraints force $\mathcal{E}_{0}$ to have the following form $\mathcal{E}_{0}=\left\{\xi \in \mathbb{R}^{2 n+q} \mid \xi^{T}(\alpha P) \xi \leq 1\right\}$.

Hence, if $\gamma$ is minimized, then $P_{0}$ is forced to be as similar as possible to a sphere; while, by decreasing $\alpha, \mathcal{E}_{0}$ is enlarged. 


\subsection{An illustrative simulation}

Consider an uncertain system defined by:

$$
\left\{\begin{array}{l}
\dot{x}(t)=(1+\beta) x(t)+u(t)+\tilde{\omega}(t), \\
y(t)=x(t),
\end{array}\right.
$$

where $\beta \in[0,2]$ is an unknown parameter that represents modeling errors and $\tilde{\omega}(t)=\sin (t)$ is an exogenous disturbance.

\subsubsection{DOBC design}

A DOBC is designed according to the following LTI disturbed model:

$$
G \triangleq\left\{\begin{array}{l}
\dot{x}(t)=x(t)+u(t)+\omega(x, \tilde{\omega}(t)), \\
y(t)=x(t) \\
z(t)=x(t)
\end{array}\right.
$$

where it can be easily seen that it corresponds to (4.12), where the uncertainty caused by the parameter $\beta$ has been translated to the disturbance signal: $\omega(\cdot) \triangleq \beta x(t)+\tilde{\omega}(t)$.

The considered DOBC is:

$$
u(t)=K_{x} \hat{x}(t)+K_{\omega, 0} \hat{\omega}(t)
$$

with $K_{x}=-2$ and $K_{\omega, 0}=-1$.

The estimates $\hat{x}(t), \hat{\omega}(t)$ are computed by the ESO (3.17a), with $r=0$ and $L=[41,400]^{T}$.

From the point of view of model (4.13), the disturbance $\omega(t)$ is clearly coupled with the nominal-state. In fact, the disturbance gets bigger as the nominalstate does. Let us consider that we want to evaluate if the closed-loop system is stable and, furthermore, if its trajectories belong to the domain $|x|<1$. Let us also check if there exist some value, $\beta_{\text {lim }}$, so that, for any $\beta>\beta_{\text {lim }}$, the closed-loop system becomes unstable. 


\begin{tabular}{|ccc|c|c|c|c|}
\hline \multicolumn{3}{|c|}{$P_{\infty}$} & $\delta_{\infty}$ & $\tau_{\infty}$ & $\gamma_{1_{\infty}}$ & $\gamma_{2_{\infty}}$ \\
\hline$\left[\begin{array}{ccc}45.4 & -248 & 2.95 \\
-248 & 151 \mathrm{e} 3 & -3.17 \mathrm{e} 3 \\
2.95 & -3.17 \mathrm{e} 3 & 81.7\end{array}\right]$ & 1.33 & 5.57 & 0 & 1.33 \\
\hline \hline & $P_{0}$ & & $\delta_{0}$ & $\tau_{0}$ & $\gamma_{1_{0}}$ & $\gamma_{2_{0}}$ \\
\hline$\left[\begin{array}{ccc}1 & 0.14 & -0.052 \\
0.14 & 13.4 & -0.70 \\
-0.052 & -0.70 & 0.15\end{array}\right]$ & 0.5 & 0.1 & 0 & 0.5 \\
\hline
\end{tabular}

Table 4.1: Optimized parameters for $\beta=2$.

\subsubsection{Application of the stability conditions}

In this case, Asm. 4.1 is satisfied with $\rho=1$ and

$$
\begin{aligned}
& \|\omega(x, \tilde{\omega}(t))\| \leq \beta+1, \quad\|\dot{\tilde{\omega}}(t)\| \leq 1 \\
& \left\|\frac{\partial \omega}{\partial x}(x, \tilde{\omega}(t))\right\| \leq \beta, \quad\left\|\frac{\partial \omega}{\partial \tilde{\omega}}(x, \tilde{\omega}(t))\right\| \leq 1,
\end{aligned}
$$

Theorem 4.2.1 is numerically optimized according to (4.10)-(4.11) and considering the worst case: $\beta=2$. The resulting sets of parameters are summarized in Table 4.1 and, with those parameters, both conditions of Thm. 4.2.1 hold. Hence, it is concluded that any trajectory starting from $\xi(0) \in \mathcal{E}_{0}$ will exponentially converge to $\mathcal{E}_{\infty}$.

A simulation result is presented in Figure 4.3, where three trajectories have been simulated for $\beta=2$ and different initial states belonging to $\mathcal{E}_{0}$. All the trajectories lie inside $\mathcal{B}_{r}$ and exponentially approach to $\mathcal{E}_{\infty}$-which is represented by the green dotted line. In fact, this behavior holds for the infinite possibilities that already exist; i.e. all the possible initial states and, in fact, any possible disturbance $\omega(x, \tilde{\omega}(t))$ that satisfies the bounds (4.15); i.e. not necessarily the specific one considered here: $\omega(x, \tilde{\omega}(t))=\beta x(t)+\tilde{\omega}(t)$.

This illustrates the usefulness of Thm. 4.2.1 for making assessments of the closed-loop behavior as -in the end- the stability result does not depend on the specific waveform of the disturbance, but just on how it could vary with respect to the nominal-state.

On the other hand, the system is stable. From the point of view of Corollary 4.1, this occurs because we are considering a "sufficiently-low" $\beta$. Henceforth, let us check if there exist some $\beta$ for which Thm. 4.2.1 becomes infeasible. 

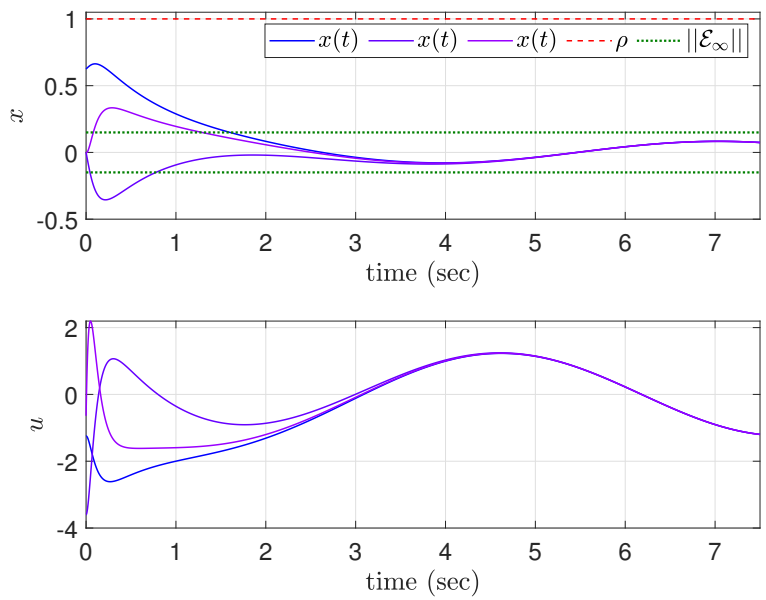

Figure 4.3: Simulation of three trajectories starting from different initial states.

To this purpose, we reproduce the same optimization procedure for increasing values of $\beta$. And it is found that, if $\beta>10.04$, it does not exist none combination of parameters $\left\{P_{i}, \delta_{i}, \tau_{i}, \gamma_{1_{i}}, \gamma_{2_{i}}\right\}$ such that the LMI (4.8) holds. This means that stability cannot be guaranteed for $\beta>10.04$. By simulations, it is found that the closed-loop becomes unstable for $\beta \geq 10.17$.

Finally, some words about the robustness introduced by the disturbance compensation term $K_{\omega, 0} \hat{\omega}(t)$ should be highlighted. The main advantage of working with LTI disturbed models is that the resulting control law depends on the disturbance itself. Thus, it helps to increase the robustness of the controller. For instance, in this case, if the disturbances are not considered -i.e. $\omega=0$; then, the resulting control law would be simply $u(t)=K_{x} x(t)=-2 x(t)$. This control law is only capable to stabilize the system for $\beta<1$. Whereas using the control law that considers the disturbance, i.e. $u(t)=-2 \hat{x}(t)-\hat{\omega}(t)$; stability is guaranteed for $\beta<10.17$. The increase on robustness achieved by considering the disturbance signal is remarkable. 


\subsection{Conclusions}

An analysis of the resulting cloose-loop formed by a DOBC and the system has been provided in this chapter.

Its aim was to highlight that one of the most important elements to guarantee a successful closed-loop behavior is the origin of the disturbance signals. Therefore, if the disturbance signals are highly coupled with the system -in the sense that a small change of the nominal system-state could cause significant changes on the observed disturbances- then the resulting closed-loop could be instabilized. Hence, when constructing the LTI disturbed model for the system, the control-designer should consider that the nominal part of the model should include the most relevant information about the system behavior, with the main purpose of minimizing the dependency between both the disturbances and the nominal system-state. 



\section{Chapter 5}

\section{DOBP: Design based on the model's solution, $x\left(t+\Delta_{t}\right)$.}

In this chapter, the synthesis of DOBPs with the structure $\hat{x}\left(t+\Delta_{t}\right)=\Phi\left(\Delta_{t}\right) \hat{x}(t)+\sum_{i=0}^{r} \Gamma_{\omega, i}\left(\Delta_{t}\right) \hat{\omega}^{(i)}(t)+\sum_{k=0}^{N} \Gamma_{u}\left(\tilde{t}_{k}, \tilde{t}_{k+1}\right) u_{k}$ -where $r \in \mathbb{N}, N \in \mathbb{N}, \tilde{t}_{i} \in \mathbb{R}, u_{k} \in \mathbb{R}^{m}$ - is presented. These DOBPs are constructed in order to approximate the time domain solution of the LTI disturbed model (2.10). Comparisons with other predictive strategies are also established.

The contents of this chapter have been published in (Castillo et al. 2020b). 


\subsection{Introduction}

The synthesis of DOBPs of the form $\hat{x}\left(t+\Delta_{t}\right)=\Phi\left(\Delta_{t}\right) \hat{x}(t)+\sum_{i=0}^{r} \Gamma_{\omega, i}\left(\Delta_{t}\right) \hat{\omega}^{(i)}(t)+$ $\sum_{k=0}^{N} \Gamma_{u}\left(\tilde{t}_{k}, \tilde{t}_{k+1}\right) u_{k}$-where $r \in \mathbb{N}, N \in \mathbb{N}, \tilde{t}_{i} \in \mathbb{R}, u_{k} \in \mathbb{R}^{m}$ is presented through this chapter. These DOBPs are constructed in order to approximate the time domain solution, $x\left(t+\Delta_{t}\right)$, of the LTI disturbed model (2.10).

For the sake of completeness, the model (2.8) and its solution (2.10) can be considered with an additional control input-delay; leading to:

$$
G \triangleq\left\{\begin{array}{l}
\dot{x}(t)=A x(t)+B_{2} u(t-h)+B_{1} \omega(t), \\
y(t)=C_{2} x(t), \\
z(t)=C_{1} x(t) .
\end{array}\right.
$$

and

$x\left(t+\Delta_{t}\right)=\Phi\left(\Delta_{t}\right) x(t)+\int_{t}^{t+\Delta_{t}} e^{A\left(t+\Delta_{t}-s\right)} B_{u} u(s-h) d s+\int_{t}^{t+\Delta_{t}} e^{A\left(t+\Delta_{t}-s\right)} B_{\omega} \omega(s) d s$,

being $e^{A \Delta_{t}} \triangleq \Phi\left(\Delta_{t}\right)$.

The insertion of the input-delay is motivated by the fact that state-predictors are highly employed to control systems with input-delays -e.g. (Krstic 2008; Krstic 2010). This delay does not really play any important role in the technical analyses of this chapter. The results are valid for $h=0$ or $h>0$.

One can easily perceive the analogy between the disturbance-dependent integral in (5.2) and the one that appeared in the analytical expression of the optimal control signal (3.9). Therefore, throughout this last chapter, the procedure that was applied in Chapter 3 in order to construct DOBCs of the form $u(t)=K_{x} \hat{x}(t)+\sum_{i=0}^{r} K_{\omega, i} \hat{\omega}^{(i)}(t)$ has been similarly employed in order to construct DOBPs of the form $\hat{x}\left(t+\Delta_{t}\right)=\Phi\left(\Delta_{t}\right) \hat{x}(t)+\sum_{i=0}^{r} \Gamma_{\omega, i}\left(\Delta_{t}\right) \hat{\omega}^{(i)}(t)+$ $\sum_{k=0}^{N} \Gamma_{u}\left(\tilde{t}_{k}, \tilde{t}_{k+1}\right) u_{k}$; with the peculiarity that the influence of the control action -i.e. $\sum_{k=0}^{N} \Gamma_{u}\left(\tilde{t}_{k}, \tilde{t}_{k+1}\right) u_{k}-$ needs to be also considered.

\section{Main assumptions and definitions}

Through this chapter, the next assumptions are considered:

Assumption 5.1. The disturbance, $\omega(t)$, is $(r+1)$-times continuously differentiable with $\left\|\omega^{(r+1)}(t)\right\| \leq \epsilon_{r} ; \forall t \in \mathbb{R}$. 
Assumption 5.2. The control action, $u(\phi)$, is piece-wise constant and it is defined for all $\phi \in\left[t-h, t+\Delta_{t}-h\right]$.

Asm. 5.1 is the same than the one taken in Chapter 3. It refers to disturbances that exhibit a certain degree of differentiability or continuity/regularity. Such property allows to predict its waveform over relatively short time-horizons.

Asm. 5.2 states that the control action is generated discretely and it is introduced to the system via a Zero-Order-Hold ( $\mathrm{ZOH})$. This is actually the case in almost all computer-based control applications (Åström et al. 2013). In addition, it says that all its jumping instants, $t_{k} \in\left[t-h, t+\Delta_{t}-h\right], k \in \mathbb{N}$, and its associated control values, $u_{k} \in \mathbb{R}^{m}$, are well-defined and, for prediction purposes, they can be considered as known. This is summarized in the following definition:

Definition 5.1. Let $t_{k} \in \mathbb{R}, k \in\{1, \ldots, N\}, N \in \mathbb{N}$; be the set of time-instants, with $t_{1}>t-h, t_{k}<t_{k+1}, t_{N}<t+\Delta_{t}-h$; such that:

$$
\begin{aligned}
& u(\phi)=u_{0} \in \mathbb{R}^{m}, \forall \phi \in\left[t-h, t_{1}\right), \\
& u(\phi)=u_{k} \in \mathbb{R}^{m}, \forall \phi \in\left[t_{k}, t_{k+1}\right), 1 \leq k \leq N-1, \\
& u(\phi)=u_{N} \in \mathbb{R}^{m}, \forall \phi \in\left[t_{N}, t+\Delta_{t}-h\right] .
\end{aligned}
$$

\subsection{Synthesizing DOBPs that approximate $x\left(t+\Delta_{t}\right)$}

In what follows, the first and second integrals in (5.2) are rewritten as the sums: $\sum_{k=0}^{N} \Gamma_{u}\left(\tilde{t}_{k}, \tilde{t}_{k+1}\right) u_{k}$ and $\sum_{i=0}^{r} \Gamma_{\omega, i}\left(\Delta_{t}\right) \hat{\omega}^{(i)}(t)$; respectively. This technical result is the key step permitting to synthesize the desired DOBPs.

On the one hand, under Asm. 5.2 and Def. 5.1, the interval $\left[t, t+\Delta_{t}\right]$ can be divided into $\mathrm{N}+1$ sub-intervals where the delayed control action remains constant. Thus:

$$
\int_{t}^{t+\Delta_{t}} e^{A\left(t+\Delta_{t}-s\right)} B_{2} u(s-h) d s=\sum_{k=0}^{N} \Gamma_{u}\left(\tilde{t}_{k}, \tilde{t}_{k+1}\right) u_{k}
$$


where, $\tilde{t}_{0}=t ; \tilde{t}_{k}=t_{k}+h$ for $k \in[1, N]$, with $t_{k}$ defined in Def. $5.1 ; \tilde{t}_{N+1}=t+\Delta_{t}$ and:

$$
\begin{aligned}
\Gamma_{u}\left(\tilde{t}_{k}, \tilde{t}_{k+1}\right) & \triangleq \int_{\tilde{t}_{k}}^{\tilde{t}_{k+1}} e^{A\left(t+\Delta_{t}-s\right)} B_{2} d s \\
& =\sum_{j=1}^{\infty}\left[\frac{\left(t+\Delta_{t}-\tilde{t}_{k}\right)^{j}-\left(t+\Delta_{t}-\tilde{t}_{k+1}\right)^{j}}{j !} A^{j-1}\right] B_{2} .
\end{aligned}
$$

On the other hand, the next lemma can be established for the disturbancedependent integral:

Lemma 5.2.1. Under Asm. 5.1:

$$
\int_{t}^{t+\Delta_{t}} e^{A\left(t+\Delta_{t}-s\right)} B_{1} \omega(s) d s=\sum_{i=0}^{r} \Gamma_{\omega, i}\left(\Delta_{t}\right) \omega^{(i)}(t)+\mathcal{R}_{r}(t) ;
$$

where $\Gamma_{\omega, i}\left(\Delta_{t}\right) \triangleq \sum_{j=0}^{\infty}\left[\frac{\Delta_{t}^{i+1+j}}{(i+1+j) !} A^{j}\right] B_{1} ;$ and

$$
\mathcal{R}_{r}(t) \triangleq \int_{t}^{t+\Delta_{t}} \frac{\left(t+\Delta_{t}-s\right)^{r+1}}{(r+1) !} \Gamma_{\omega, r}\left(t+\Delta_{t}-s\right) B_{1} \omega^{(r+1)}(s) d s .
$$

Proof. Let $v_{i} \triangleq \int_{t}^{t+\Delta_{t}} A^{-i} e^{A\left(t+\Delta_{t}-s\right)} B_{1} \omega^{(i)}(s) d s$. Under Ass. 5.1, $v_{i}$ can be integrated by parts for any $i<r+1$; leading to:

$$
v_{i}=-A^{-(i+1)} B_{1} \omega^{(i)}\left(t+\Delta_{t}\right)+A^{-(i+1)} e^{A \Delta_{t}} B_{1} \omega^{(i)}(t)+v_{i+1}
$$

The recursive Eq. (5.7) allows to express $v_{0}$ as:

$$
\begin{aligned}
v_{0}=-\left[A^{-1} B_{1} \omega\left(t+\Delta_{t}\right)+\ldots+A^{-(r+1)} B_{1} \omega^{(r)}\left(t+\Delta_{t}\right)\right] \\
+\left[A^{-1} e^{A \Delta_{t}} B_{1} \omega(t)+\ldots+A^{-(r+1)} e^{A \Delta_{t}} B_{1} \omega^{(r)}(t)\right]+v_{r+1} .
\end{aligned}
$$

Let $h_{i} \triangleq \int_{t}^{t+\Delta_{t}} \frac{\left(t+\Delta_{t}-s\right)^{i}}{i !} \omega^{(r+1)}(s) d s$. By Taylor, it holds that:

$$
\begin{aligned}
& \omega\left(t+\Delta_{t}\right)=\omega(t)+\Delta_{t} \dot{\omega}(t)+\ldots+\frac{\Delta_{t}^{r}}{r !} \omega^{(r)}(t)+h_{r} \\
& \dot{\omega}\left(t+\Delta_{t}\right)=\dot{\omega}(t)+\Delta_{t} \ddot{\omega}(t)+\ldots+\frac{\Delta_{t}^{r-1}}{(r-1) !} \omega^{(r)}(t)+h_{r-1} \\
& \quad \vdots \\
& \omega^{(r)}\left(t+\Delta_{t}\right)=\omega^{(r)}(t)+h_{0}
\end{aligned}
$$


Thus, by substituting (5.9) into (5.8), and after rearranging terms, the integral $v_{0}$ results in:

$$
\begin{aligned}
v_{0}= & {\left[-A^{-1}+A^{-1} e^{A \Delta_{t}}\right] B_{1} \omega(t) } \\
& +\left[-A^{-2}-\Delta_{t} A^{-1}+A^{-2} e^{A \Delta_{t}}\right] B_{1} \omega^{(1)}(t) \\
& \vdots \\
& +\left[-A^{-(r+1)}-\Delta_{t} A^{-r}-\frac{\Delta_{t}^{2}}{2 !} A^{-r-1}-\ldots-\frac{\Delta_{t}^{r}}{r !} A^{-1}+A^{-(r+1)} e^{A \Delta_{t}}\right] B_{1} \omega^{(r)}(t) \\
& +v_{r+1}-A^{-1} B_{1} h_{r}-\ldots-A^{-(r+1)} B_{1} h_{0} .
\end{aligned}
$$

Equality (5.5) follows by: i) noting that $v_{0}$ equals to the integral in the LHS of (5.5); and ii) expressing $e^{A \Delta_{t}}$ as power-series and canceling the terms that are added and subtracted in each term of the RHS of (5.10).

The equalities (5.3), (5.5) enable to immediately prove the following theorem; which states how to construct the desired DOBPs:

Theorem 5.2.1. Under Assumptions 5.1-5.2, let $t+\Delta_{t}, \Delta_{t} \geq 0$, be a future time-instant. Consider the set of control inputs, $\boldsymbol{u}_{\mathbf{0}-\boldsymbol{N}} \triangleq\left[u_{0}^{T}, u_{1}^{T}, \ldots, u_{N}^{T}\right]^{T}$, of Definition 5.1 that have been, or could be ${ }^{1}$, introduced to the system during the time-interval $\left[t-h, t+\Delta_{t}-h\right]$. Then, with any estimations, $\hat{x}(t), \hat{\omega}_{r}(t)$, of the variables $x(t)$ and $\omega_{\boldsymbol{r}}(t) \triangleq\left[\omega^{T}(t), \dot{\omega}^{T}(t), \ldots, \omega^{(r) T}(t)\right]^{T}$ in $(2.8)$, respectively; the future-state, $x\left(t+\Delta_{t}\right)$, can be predicted by:

$$
\hat{x}\left(t+\Delta_{t}\right)=\Phi\left(\Delta_{t}\right) \hat{x}(t)+\boldsymbol{\Gamma}_{\boldsymbol{\omega}}\left(\Delta_{t}\right) \hat{\boldsymbol{\omega}}_{\boldsymbol{r}}(t)+\boldsymbol{\Gamma}_{\boldsymbol{u}}\left(\tilde{t}_{0}, \ldots, \tilde{t}_{N+1}\right) \boldsymbol{u}_{\mathbf{0}-\boldsymbol{N}}
$$

with an error equal to

$$
x\left(t+\Delta_{t}\right)-\hat{x}\left(t+\Delta_{t}\right)=\mathcal{R}_{e}(t)+\mathcal{R}_{r}(t)
$$

being:

$$
\begin{aligned}
& \boldsymbol{\Gamma}_{\boldsymbol{\omega}}\left(\Delta_{t}\right) \triangleq\left[\Gamma_{\omega, 0}\left(\Delta_{t}\right), \Gamma_{\omega, 1}\left(\Delta_{t}\right), \ldots, \Gamma_{\omega, r}\left(\Delta_{t}\right)\right] \\
& \boldsymbol{\Gamma}_{\boldsymbol{u}}\left(\tilde{t}_{0}, \ldots, \tilde{t}_{N+1}\right) \triangleq\left[\Gamma_{u}\left(\tilde{t}_{0}, \tilde{t}_{1}\right), \Gamma_{u}\left(\tilde{t}_{1}, \tilde{t}_{2}\right), \ldots, \Gamma_{u}\left(\tilde{t}_{N}, \tilde{t}_{N+1}\right)\right], \\
& \mathcal{R}_{e}(t) \triangleq e^{A T}[x(t)-\hat{x}(t)]+\boldsymbol{\Gamma}_{\boldsymbol{\omega}}\left(\Delta_{t}\right)\left[\boldsymbol{\omega}_{\boldsymbol{r}}(t)-\hat{\boldsymbol{\omega}}_{\boldsymbol{r}}(t)\right], \\
& \mathcal{R}_{r}(t) \triangleq \int_{t}^{t+\Delta_{t}} \frac{\left(t+\Delta_{t}-s\right)^{r+1}}{(r+1) !} \Gamma_{\omega, r}\left(t+\Delta_{t}-s\right) B_{1} \omega^{(r+1)}(s) d s ;
\end{aligned}
$$

where $\Gamma_{\omega, i}\left(\Delta_{t}\right)$ and $\Gamma_{u}\left(\tilde{t}_{k}, \tilde{t}_{k+1}\right)$ are defined in (5.5) and (5.4), respectively.

${ }^{1}$ Note that, if $\Delta_{t}>h$, then Def. 5.1 includes a set of future control actions that could be introduced to the system. Those control actions, needless to say, affect to the future state. 
Proof. The proof follows by substituting (5.3), (5.5) into (5.2) and subtracting (5.11) from it.

Thm. 5.2.1 closely resembles to Thm. 3.3.1 in Chapter 3; but, in this case, the theorem permits to predict the future-state $x\left(t+\Delta_{t}\right)$ by the DOBs. As mentioned in Sec. 2.2, the existing literature on DOBs has provided with a wide number of efficient algorithms and numerical methods for estimating -in real-time- the variables $x(t)$ and $\boldsymbol{\omega}_{\boldsymbol{r}}(t)$, in (3.10), through the historical set of sensor readings $y(t)$. Some examples are the ones by Luenberger 1964; Luenberger 1966; Schweppe 1968; Meditch et al. 1973; Bhattacharyya 1978; Darouach et al. 1994; Corless et al. 1998; Xiong et al. 2003; Chang 2006; Kim et al. 2010; Chakrabarty et al. 2017; Su et al. 2018. Any of these DOBs could be combined with Thm. 5.2.1 in order to produce a DOBP that predicts the future-state $x\left(t+\Delta_{t}\right)$.

\section{Resulting prediction errors}

The prediction $\hat{x}\left(t+\Delta_{t}\right)$ by Thm. 5.2.1 is subject to unavoidable errors -i.e. $\mathcal{R}_{e}(t)$ and $\mathcal{R}_{r}(t)$ - which appear as a direct consequence of not having all the necessary information in order to directly compute the future state $x\left(t+\Delta_{t}\right)$.

These errors are of the same nature than the ones in Thm. 3.3.1: i) $\mathcal{R}_{e}(t)$, which accounts for the current estimation errors, $x(t)-\hat{x}(t), \boldsymbol{\omega}_{r}(t)-\hat{\boldsymbol{\omega}}_{r}(t)$; and ii) $\mathcal{R}_{r}(t)$, which, under perfect observation conditions (i.e. $\mathcal{R}_{e}=0$ ), represents the error caused by predicting $x\left(t+\Delta_{t}\right)$ with only the actual disturbance state, $\boldsymbol{\omega}_{r}(t)$, instead of computing it with the whole disturbance function, $\omega:\left[t, t+\Delta_{t}\right] \rightarrow \mathbb{R}^{q}$. This represents the unavoidable error appearing as a consequence of not knowing the future disturbance behavior.

By taking norms on $(5.6), \mathcal{R}_{r}(t)$ can be bounded by:

$$
\left\|\mathcal{R}_{r}(t)\right\| \leq \frac{\Delta_{t}^{r+2}}{(r+2) !} \mu \epsilon_{r}
$$

where $\mu \triangleq \max _{s \in\left[t, t+\Delta_{t}\right]}\left\{\left\|\Gamma_{\omega, r}\left(t+\Delta_{t}-s\right) B_{1}\right\|\right\}$.

It is seen that the size of $\mathcal{R}_{r}(t)$ mainly depends on the prediction horizon as well as on the $(r+1)$ disturbance-derivative bound -defined in Asm. 5.1. This bound mainly shows that the prediction error rapidly increases as $\Delta_{t}$ grows; whereas it approaches to zero as $\Delta_{t} \rightarrow 0$. In this sense, accurate predictions cannot be expected for excessively long time-horizons; but it is not the case 
for relative-short time-horizons, where accurate predictions could in fact be obtained.

On the other hand, the term $\mathcal{R}_{e}(t)$ entirely depends on how the observations are computed. It mainly depends on the capability of the DOB to give accurate estimates of $x(t)$ and $\boldsymbol{\omega}_{\boldsymbol{r}}(t)$. Thus, accurate observations should be required in order to reduce this term.

\subsection{Application: design of a high-order ESO-based predictor}

In the same way as it has been displayed in Chapter 3, let us show how a high-order Extended State Observer (ESO)-based predictor could be easily synthesized with this new methodology. The ESOs are, nowadays, one of the most popular algorithms to estimate in real-time the system and disturbance states due to their simplicity -e.g. (Castillo et al. 2018; Chen et al. 2016; Chang 2006). Let us show how they could be employed under this novel feedback design.

Let us consider the following high-order ESO:

$$
\begin{aligned}
& \dot{\hat{\eta}}(t)=(\bar{A}-L \bar{C}) \hat{\eta}(t)+\bar{B}_{u} u(t-h)+L y(t), \\
& \xi(t)=\Psi\left(\Delta_{t}\right) \hat{\eta}(t)
\end{aligned}
$$

being $\hat{\eta} \in \mathbb{R}^{l} ; \bar{A}, \bar{C}$ and $\bar{B}_{u}$ defined in $(3.17) ; \Psi\left(\Delta_{t}\right) \triangleq\left[\Phi\left(\Delta_{t}\right), \boldsymbol{\Gamma}_{\boldsymbol{\omega}}\left(\Delta_{t}\right)\right] ;$ and $L \in \mathbb{R}^{l \times p}$ a free-design matrix.

As can be seen in Sec. 3.4, Eq. (3.17a) is a conventional high-order ESO for the augmented system $\eta(t) \triangleq\left[x^{T}(t), \boldsymbol{\omega}_{\boldsymbol{r}}^{T}(t)\right]^{T}$; whose dynamics -under Asm. 3.2is given by:

$$
\dot{\eta}(t)=\bar{A} \eta(t)+\bar{B}_{2} u(t)+\bar{B}_{1} \omega^{(r+1)}(t),
$$

with $\bar{B}_{1} \triangleq\left[0_{q \times(n+r q)}, I_{q}\right]^{T}$.

It, thus, provides with the needed estimates $\hat{x}(t), \hat{\boldsymbol{\omega}}_{\boldsymbol{r}}(t)$ to implement Eq. (3.17a) -which has been inserted in (3.17b).

By Thm. 5.2.1, the output of (5.14) can be employed to predict the future state $x\left(t+\Delta_{t}\right)$ as follows:

$$
\hat{x}\left(t+\Delta_{t}\right)=\xi(t)+\boldsymbol{\Gamma}_{\boldsymbol{u}}\left(\tilde{t}_{0}, \ldots, \tilde{t}_{N+1}\right) \boldsymbol{u}_{\mathbf{0}-\boldsymbol{N}} ;
$$

where the second term in the RHS quantifies for the effect of the control action. 
In addition, the error $\mathcal{R}_{e}(t)$ in (5.12) is defined by:

$$
\begin{aligned}
\dot{e}_{\eta}(t) & =(\bar{A}-L \bar{C}) e_{\eta}(t)+\bar{B}_{\omega} \omega^{(r+1)}(t), \\
\mathcal{R}_{e}(t) & =\Psi\left(\Delta_{t}\right) e_{\eta}(t) .
\end{aligned}
$$

being $e_{\eta} \triangleq \eta(t)-\hat{\eta}(t)$.

Just as it is appreciated in Sec. 3.4, the gain $L$ could be tuned through Eq. (3.19) in order to explicitly minimize $\mathcal{R}_{e}(t)$. This can be done by standard numerical methods, such as Lyapunov-based convex-optimization, $\mathcal{H}_{\infty}$ designs (Scherer et al. 2000), or the LMI-procedure of Lemma 3.4.1 - where the constraint (3.20b) should be replaced by $P-\alpha \Psi^{T}\left(\Delta_{t}\right) \Psi\left(\Delta_{t}\right) \succeq 0$.

\subsection{Comparative example}

Some numerical simulations and comparisons with respect to the predictors that were previously reported by Léchappé et al. 2015 and Sanz et al. 2016a are included in this section.

This section contains numerical simulations and comparisons with respect to the predictors that were previously reported by Léchappé et al. 2015 and Sanz et al. 2016a.

These works gave alternative solutions to this future-state prediction problem in LTI disturbed systems. Table 5.1 contains a brief comparison of each method, showing how the predictions are computed. In this table, it can be seen that the predictor by Léchappé et al. 2015 is the most easily implementable as it does not need to implement any observer. This strategy is based on replacing the disturbance-dependent integral of the system solution (5.2) by an error-term that compares the actual state, $x(t)$, with the prediction, $x_{p}(t) \triangleq e^{A T} x(t)+\int_{t}^{t+\Delta_{t}} e^{A\left(t+\Delta_{t}-s\right)} B_{2} u(s-h) d s$, that was made at $\left(t-\Delta_{t}\right)$. With this method, null prediction error is assured for constant disturbances. Conversely, the predictor by Sanz et al. 2016b was originally developed in order to compensate for the effect of matched disturbances in systems with input delays. It implements an observer of order $(r+1) q$ in order to predict $\bar{\omega}(t) \approx \omega\left(t+\Delta_{t}\right)$. This future-disturbance prediction is then used to approximate the disturbance-dependent integral by: $\int_{t-\Delta_{t}}^{t} e^{A(t-s)} B_{1} \bar{\omega}(s) d s$; which can be numerically computed -e.g. by the trapezoidal or Newton-Cote methodsas it just depends on the past values of the observed variable. 


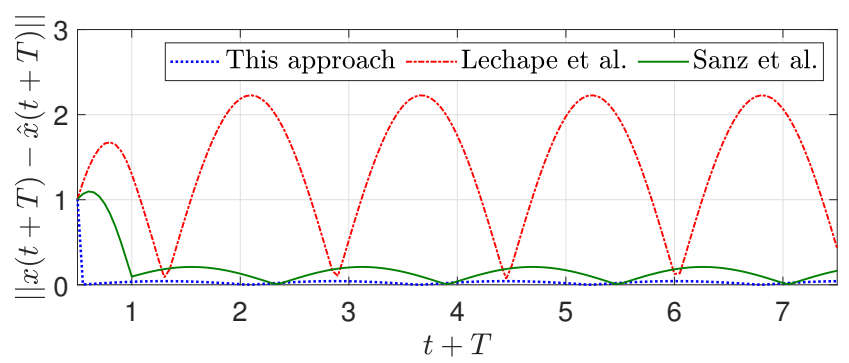

Figure 5.1: Prediction errors for $\Omega=2 \mathrm{rad} / \mathrm{s}$ and $\Delta_{t}=h=0.5$.

In contrast, the solution given here permits to construct a DOB that directly estimates $\xi(t)=\Phi\left(\Delta_{t}\right) \hat{x}(t)+\boldsymbol{\Gamma}_{\boldsymbol{\omega}}\left(\Delta_{t}\right) \hat{\boldsymbol{\omega}}_{\boldsymbol{r}}(t) \approx e^{A T} x(t)+\int_{t}^{t+\Delta_{t}} e^{A\left(t+\Delta_{t}-s\right)} B_{1} \omega(s) d s$. This leads to significant advantages in terms of implementation simplicity, computation effort and, also, prediction accuracy -as shown in the following section.

\section{Numerical simulation}

Let us evaluate the proposed DOBP with the same example considered by Léchappé et al. 2015; Sanz et al. 2016a. Consider the following LTI disturbed model:

$$
\dot{x}(t)=\left[\begin{array}{cc}
0 & 1 \\
-9 & 3
\end{array}\right] x(t)+\left[\begin{array}{l}
0 \\
1
\end{array}\right] u(t-h)+\left[\begin{array}{l}
0 \\
1
\end{array}\right] \omega(t),
$$

with $\omega(t)=3 \sin (\Omega t) ; \Omega \geq 0(\mathrm{rad} / \mathrm{s}) ;$ and $x(0)=0$.

An accurate state-prediction, $\hat{x}\left(t+\Delta_{t}\right)$, with $\Delta_{t}=h$, is required in order to approximately implement a stabilizing feedback with FSA:

$$
u(t)=-[45,18] \hat{x}\left(t+\Delta_{t}\right) .
$$

\begin{tabular}{|c|cc|}
\hline & observed variable & computed prediction \\
\hline $\begin{array}{c}\text { Léchappé } \\
\text { et al. }\end{array}$ & - & $\hat{x}\left(t+\Delta_{t}\right)=x_{p}(t)$ \\
Sanz et al. & $\bar{\omega}(t) \approx \omega\left(t+\Delta_{t}\right)$ & $\hat{x}(t)-x_{p}\left(t-\Delta_{t}\right)$ \\
This & $\left.\xi(t) \approx \Delta_{t}\right)=x_{p}(t)$ \\
approach & $\int_{t}^{t+\Delta_{t}} e^{A\left(t+\Delta_{t}-s\right)} B_{1} \omega(s) d s$ & $+\int_{t-\Delta_{t}}^{t} e^{A(t-s)} B_{1} \bar{\omega}(s) d s$ \\
\end{tabular}

Table 5.1: Comparison of predictors; with $x_{p}(t) \triangleq e^{A T} x(t)+\int_{t}^{t+\Delta_{t}} e^{A\left(t+\Delta_{t}-s\right)} B_{2} u(s-h) d s$. 


\begin{tabular}{|c|ccc|}
$\Omega$ & This approach & Léchappé et al. & Sanz et al. \\
\hline 0.5 & $1.7 \mathrm{e}-4$ & 0.60 & $8.9 \mathrm{e}-4$ \\
2 & 0.043 & 2.23 & 0.21 \\
4 & 0.66 & 3.47 & 2.82 \\
\hline
\end{tabular}

Table 5.2: $\max _{t+\Delta_{t} \in\left[\Delta_{t}, \infty\right]}\left\{\left\|x\left(t+\Delta_{t}\right)-\hat{x}\left(t+\Delta_{t}\right)\right\|\right\}$ for $\Delta_{t}=h=0.5$.

\begin{tabular}{|c|ccc|}
$\Delta_{t}$ & This approach & Léchappé et al. & Sanz et al. \\
\hline 0.5 & $1.7 \mathrm{e}-4$ & 0.60 & $8.9 \mathrm{e}-4$ \\
1 & $3.8 \mathrm{e}-3$ & 1.66 & 0.013 \\
2 & 0.072 & 21.03 & 1.12 \\
\hline
\end{tabular}

Table 5.3: $\max _{t^{\prime} \in\left[\Delta_{t}, 15\right]}\left\{\left\|x\left(t^{\prime}\right)-\hat{x}\left(t^{\prime}\right)\right\|\right\}$ for $\Omega=0.5$.

The control action is computed by (5.19) and, in order to satisfy Asm. 5.2, it is introduced to the system via a $\mathrm{ZOH}$ with period $0.05 \mathrm{sec}$.

The three predictors in Table 5.3 have been implemented in order to compute $\hat{x}\left(t+\Delta_{t}\right)$. Concretely, the proposed solution has been implemented by means of the high-order ESO (5.14), (5.16); starting from $\hat{\eta}(0)=\eta(0)$ and with $L=[10.28,4.32,512,9680,1.16 \mathrm{e} 05,7.96 \mathrm{e} 05 ; 0.22,58.8,2960,83900,1.5 \mathrm{e} 06$, $1.38 \mathrm{e} 07]$ - resulting from the optimization of the LMI in Lem. 3.4.1, with $r=3, \mathcal{D}$ a disk centered at $(0,0)$ with radius 40 and $\delta \alpha=6.87 \mathrm{e} 05$. For fair comparison, the variable $\bar{\omega}(t) \approx \omega\left(t+\Delta_{t}\right)$ of Sanz et al. 2016a has been computed by Taylor with the same estimate $\hat{\boldsymbol{\omega}}_{\boldsymbol{r}}(t)$ given by $(5.14 \mathrm{a})$; while $\int_{t-\Delta_{t}}^{t} e^{A(t-s)} B_{1} \bar{\omega}(s) d s$ has been numerically solved by the trapezoidal method with 100 intervals.

Tables 5.2-5.3 and Fig. 5.1 contain the resulting prediction errors with each method for different disturbance frequencies and different prediction-horizons. It can be appreciated that this result leads to significant improvements in terms of accuracy in all the considered frequencies and prediction-horizons. 


\subsection{Conclusions}

Along this chapter, it has been shown how to synthesize DOBPs that approximately implement the time-domain solution of an LTI disturbed model. The proposed DOBPs are easily implementable and they yield to reduced errors if compared with other previously published approaches. 



\section{Conclusions and future works}

In this thesis, new strategies to design robust Disturbance Observer-Based Controllers (DOBCs) and State-Predictors (DOBPs) for uncertain dynamic systems have been developed.

The novel DOBCs are mainly summarized in Theorem 3.3.1. They have been constructed with the main purpose of approximating the unrealizable optimal control signal for the LTI disturbed model that was defined in Sec. 2.3. Similarly, the DOBPs are summarized in Theorem 5.2.1 and they have been constructed to approximate the temporal solution of the LTI disturbed model.

At the end, these results could be understood as a tool-box that is easily implementable in MatLab ${ }^{\circledR}$ or Python ${ }^{\circledR}$. The toolbox receives as inputs a generalized LTI disturbed model -plus some intuitive tuning-parameters; i.e. $Q, R, \gamma$, $r, \Delta_{t^{-}}$and it automatically produces as an output a DOBC or a DOBP; which are computed according to the equations (3.10) or (5.11), respectively. Some MatLab ${ }^{\circledR}$ functions implementing these results can be found in the Annex 2. Also, the main code that was run in the simulations can be downloaded from: https : //github.com/alcasfra/New-strategies-to-design-DOBCs-and-DOBPs.git

From a theoretical perspective, the results of this thesis give a new framework to design DOBCs; whose major advantages are that it is indistinctly valid for SISO/MIMO models with single/multiple matched/mismatched disturbances and that it offers a highly intuitive and versatile tuning through the input parameters. In addition, these results have established some interesting con- 
nections between the optimal control theory and the robust DOBC approach; which may lead to future works/discussions.

To fix and define the framework of this research, Chapter 1 and Chapter 2 have been focused on illustrating what is understood by an uncertain dynamic system and how it can be mathematically represented by LTI disturbed models. These chapters may provide deeper insights into the physical meaning of the main variables on which the DOBCs and DOBPs are expressed. Finally, Chapter 4 has analyzed the closed-loop stability of the DOBCs in order to highlighting that some stability/performance problems may appear if the disturbance signals are highly coupled with the nominal system-state.

\section{Publications, collaborations and future works derived from this thesis}

As a consequence of this thesis, different publications have been written. Most of them have been already published -i.e. (Castillo et al. 2016; Castillo et al. 2017; Castillo et al. 2018; Castillo et al. 2019a; Castillo et al. 2019b; Castillo et al. 2020b; Castillo et al. 2020d) - and other ones are currently under editorial review -i.e. (Castillo et al. 2020a; Castillo et al. 2020c).

In addition, this dissertation has given risen to some collaborations with other research groups. The first one took place in China, at the Zhejiang University (ZJU), Hangzhou. It was a 5-weeks research stay during September, 2017, working together with prof. Chao $\mathrm{Xu}$ in drone control algorithms and highprecision RTK-GPS positioning.

A second collaboration at the Universidades Federal de Santa Catarina (UFSC), Florianopolos, Brazil began at the beginning of 2019. This collaboration consisted on a three-months research stay cooperating with prof. Julio Elias Normey-Rico. The main objective was to extend some of the methods developed through this work -concretely, the ones of Chapter 3- to models that contain actuators saturations and other restrictions. The publication (Castillo et al. 2020d) has been written together with the brazilian group and it goes in this line. Currently, some enhancements are being explored.

Subsequently, at the end of 2019 a third collaboration began at the Heudyasic Laboratory (UMR CNRS UTC 7253), Compiegne, France. It was a threemonths research stay working together with prof. Pedro Castillo. The main purpose was to experimentally apply the DOBPs developed in Chapter 5 in order to construct collision avoidance algorithms for aerial vehicles moving 
in uncertain environments. The idea was that the aerial vehicles employ the DOBP reported here in order to estimate the future trajectories that other nearby vehicles with uncertain motion will follow. Thus, if the predictor detects an imminent collision, the vehicle reacts to avoid it. Successful experimental results where carried out and sent for publication in Castillo et al. 2020a. The door on this line is still opened as these type of algorithms and collision-avoidance strategies may become a daily reality with the advent of the 5G technology (Ruggiero et al. 2018; Strategic research agenda for robotics in Europe 2014-2020).

Finally, a recent collaboration with the project Tecnodiabetes ai2, UPV, coordinated by prof. Jorge Bondia, has started. The main objective is to perform further tests of the DOBCs reported here to the problem of closed-loop glucose-control for diabetic people - which was also mentioned before. Some preliminary simulations showed satisfactory results. 



\section{Bibliography}

Albertos, Pedro and Iven Mareels (2010). Feedback and control for everyone. Springer Science \& Business Media (cit. on pp. 20, 24).

Artstein, Zvi (1982). "Linear systems with delayed controls: A reduction". In: IEEE Transactions on Automatic control 27.4, pp. 869-879 (cit. on pp. 8, 21).

Åström, Karl J and Björn Wittenmark (2013). Computer-controlled systems: theory and design. Courier Corporation (cit. on p. 65).

Åström, Karl Johan and Panganamala Ramana Kumar (2014). "Control: A perspective." In: Automatica 50.1, pp. 3-43 (cit. on p. 19).

Athans, M (1971). "On the LQG problem". In: IEEE Transactions on Automatic Control 16.6, pp. 528-528 (cit. on p. 3).

Başar, Tamer and Geert Jan Olsder (1998). Dynamic noncooperative game theory. SIAM (cit. on pp. 5, 20).

Bernardi, Emanuel and Eduardo J Adam (2020). "Observer-based fault detection and diagnosis strategy for industrial processes". In: Journal of the Franklin Institute 357.14, pp. 10054-10081 (cit. on pp. 5, 6, 18). 
Bernhard, Pierre (2003). "A robust control approach to option pricing". In: Applications of Robust Decision Theory and Ambiguity in Finance. City University Press, London (cit. on p. 5).

Bhattacharyya, S (1978). "Observer design for linear systems with unknown inputs". In: IEEE transactions on Automatic Control 23.3, pp. 483-484 (cit. on pp. 4, 19, 33, 68).

Binder, BJT, TA Johansen, and L Imsland (2019). "Improved predictions from measured disturbances in linear model predictive control". In: Journal of Process Control 75, pp. 86-106 (cit. on pp. 8, 21).

Bünte, Tilman, Dirk Odenthal, Bilin Aksun-Güvenç, and Levent Güvenç (2002). "Robust vehicle steering control design based on the disturbance observer". In: Annual reviews in control 26.1, pp. 139-149 (cit. on p. 5).

Castillo, A, P García, R Sanz, and P Albertos (2018). "Enhanced extended state observer-based control for systems with mismatched uncertainties and disturbances". In: ISA transactions 73 , pp. 1-10 (cit. on pp. 6, 7, 11, $12,18,34,69,74)$.

Castillo, A, R Sanz, P Garcia, and P Albertos (2017). "Robust design of the uncertainty and disturbance estimator". In: The 20th world congress of the International Federation of Automatic Control. IFAC, pp. 8262-8267 (cit. on pp. 12, 49, 74).

Castillo, A, Ricardo Sanz, Pedro Garcia, Wei Qiu, Hongda Wang, and Chao Xu (2019a). "Disturbance observer-based quadrotor attitude tracking control for aggressive maneuvers". In: Control Engineering Practice 82, pp. 14-23 (cit. on pp. 5, 9, 12, 24, 51, 74).

Castillo, Alberto, Jossuè Cariño, Pedro Castillo, and Pedro Garcia (2020a). "A reactive control scheme for evasive maneuvers in aerial robots". In: Robotics and Autonomous Systems (cit. on pp. 12, 21, 74, 75).

Castillo, Alberto and Pedro Garcia (2020b). "Predicting the future state of disturbed LTI systems: A solution based on high-order observers." In: Automatica (cit. on pp. 6, 11, 12, 18, 34, 36, 63, 74). 
Castillo, Alberto, Pedro Garcia, and Emilia Fridman (2020c). "Disturbance Observer-Based Control: a Novel Feedback Design-Strategy". In: (cit. on pp. 11, 12, 74).

Castillo, Alberto, Pedro García, Emilia Fridman, and Pedro Albertos (2019b). "Extended state observer-based control for systems with locally Lipschitz uncertainties: LMI-based stability conditions". In: Systems \& Control Letters 134 (cit. on pp. 12, 31, 40, 49, 74).

Castillo, Alberto, Tito L.M. Santos, Pedro Garcia, and Julio E. Normey-Rico (2020d). "Predictive ESO-based control with guaranteed stability for complex uncertain constrained systems". In: ISA transactions (cit. on pp. 11, $12,74)$.

Castillo, Alberto, Ricardo Sanz, Pedro Garcia, and Pedro Albertos (2016). "A quaternion-based and active disturbance rejection attitude control for quadrotor". In: International Conference on Information and Automation (ICIA). IEEE, pp. 240-245 (cit. on pp. 9, 12, 74).

Chakrabarty, Ankush, Martin J Corless, Gregery T Buzzard, Stanisław H Żak, and Ann E Rundell (2017). "State and unknown input observers for nonlinear systems with bounded exogenous inputs". In: IEEE Transactions on Automatic Control 62.11, pp. 5497-5510 (cit. on pp. 19, 33, 68).

Chang, Jeang-Lin (2006). "Applying discrete-time proportional integral observers for state and disturbance estimations". In: IEEE Transactions on Automatic Control 51.5, pp. 814-818 (cit. on pp. 19, 33, 34, 68, 69).

Chapellat, Hervé and SP Bhattacharyya (1989). "A generalization of Kharitonov's theorem; Robust stability of interval plants". In: IEEE transactions on automatic control 34.3, pp. 306-311 (cit. on p. 3).

Chen, Wen Hua, Jun Yang, Lei Guo, and Shihua Li (2016). "Disturbanceobserver-based control and related methods-An overview". In: IEEE Transactions on Industrial Electronics 63.2, pp. 1083-1095 (cit. on pp. 4, $6,7,9,10,19,24,34,40,69)$.

Chilali, Mahmoud and Pascal Gahinet (1996). " $\mathcal{H}_{\infty}$ design with pole placement constraints: an LMI approach". In: IEEE Transactions on automatic control 41.3, pp. 358-367 (cit. on pp. 35, 98). 
Chilali, Mahmoud, Pascal Gahinet, and Pierre Apkarian (1999). "Robust pole placement in LMI regions". In: IEEE transactions on Automatic Control 44.12 , pp. 2257-2270 (cit. on pp. 35, 98).

Choi, Byeong-Kap, Chong-Ho Choi, and Hyuk Lim (1999). "Model-based disturbance attenuation for CNC machining centers in cutting process". In: IEEE/ASME transactions on mechatronics 4.2, pp. 157-168 (cit. on p. 5).

Corless, Martin and JAY Tu (1998). "State and input estimation for a class of uncertain systems". In: Automatica 34.6, pp. 757-764 (cit. on pp. 19, 33, $68)$.

Darouach, Mohamed, Michel Zasadzinski, and Shi Jie Xu (1994). "Full-order observers for linear systems with unknown inputs". In: IEEE transactions on automatic control 39.3, pp. 606-609 (cit. on pp. 4, 19, 33, 68).

Deng, Jiuqiang, Wenchao Xue, Xi Zhou, and Yao Mao (2020). "On disturbance rejection control for inertial stabilization of long-distance laser positioning with movable platform". In: Measurement and Control (cit. on p. 5).

Di Loreto, Michael, JF Lafay, and JJ Loiseau (2005). "Disturbance attenuation by dynamic output feedback for input-delay systems". In: Proceedings of the 44th IEEE Conference on Decision and Control. IEEE, pp. 5042-5047 (cit. on pp. 8, 21).

Di Ruscio, David (1992). "On the location of LQ-optimal closed-loop poles". In: (cit. on p. 34).

Doyle, J. C., K. Glover, P. P. Khargonekar, and B. A. Francis (1989). "Statespace solutions to standard $\mathrm{H} /$ sub $2 /$ and $\mathrm{H} /$ sub infinity / control problems". In: IEEE Transactions on Automatic Control 34.8, pp. 831-847 (cit. on pp. 4, 19, 23).

Doyle, John, Keith Glover, Pramod Khargonekar, and Bruce Francis (1988). "State-space solutions to standard $\mathcal{H}_{2}$ and $\mathcal{H}_{\infty}$ control problems". In: 1988 American Control Conference. IEEE, pp. 1691-1696 (cit. on p. 4).

Doyle, John C (1978). "Guaranteed margins for LQG regulators". In: IEEE Transactions on automatic Control 23.4, pp. 756-757 (cit. on p. 3). 
Edwards, Christopher and Sarah Spurgeon (1998). Sliding mode control: theory and applications. Crc Press (cit. on p. 4).

Francis, Bruce A and Walter Murray Wonham (1976). "The internal model principle of control theory". In: Automatica 12.5, pp. 457-465 (cit. on p. 4).

Freeman, Randy and Petar V Kokotovic (2008). Robust nonlinear control design: state-space and Lyapunov techniques. Springer Science \& Business Media (cit. on p. 3).

Fridman, Emilia (2014). Introduction to time-delay systems: Analysis and control. Springer (cit. on p. 54).

Fujimoto, Hiroshi, Takeo Saito, and Toshihiko Noguchi (2004). "Motion stabilization control of electric vehicle under snowy conditions based on yawmoment observer". In: The 8th IEEE International Workshop on Advanced Motion Control, 2004. AMC'04. IEEE, pp. 35-40 (cit. on p. 5).

Furtat, Igor, Emilia Fridman, and Alexander Fradkov (2017). "Disturbance compensation with finite spectrum assignment for plants with input delay". In: IEEE Transactions on Automatic Control 63.1, pp. 298-305 (cit. on pp. 8, 21).

Gahinet, Pascal and Pierre Apkarian (1994). "A linear matrix inequality approach to H/sub infinity / control". In: International journal of robust and nonlinear control 4.4, pp. 421-448 (cit. on p. 23).

Gao, Zhiqiang (2006). "Active disturbance rejection control: a paradigm shift in feedback control system design". In: 2006 American control conference. IEEE, 7-pp (cit. on pp. 4, 51).

- (2014). "On the centrality of disturbance rejection in automatic control". In: ISA transactions 53.4, pp. 850-857 (cit. on pp. 2, 28).

Gao, Zhiqiang, Yi Huang, and Jingqing Han (2001). "An alternative paradigm for control system design". In: Proceedings of the 40th IEEE conference on decision and control (Cat. No. 01CH37228). Vol. 5. IEEE, pp. 4578-4585 (cit. on p. 4). 
Garcia, Carlos E and Manfred Morari (1982). "Internal model control. A unifying review and some new results". In: Industrial \&3 Engineering Chemistry Process Design and Development 21.2, pp. 308-323 (cit. on p. 4).

Gelfand, Izrail Moiseevitch and S. V. Fomin (2000). Calculus of variations. Courier Corporation (cit. on p. 29).

Guo, Lei and Songyin Cao (2014). "Anti-disturbance control theory for systems with multiple disturbances: A survey". In: ISA transactions 53.4, pp. 846849 (cit. on pp. 6, 7).

Guo, Lei and Wen-Hua Chen (2005). "Disturbance attenuation and rejection for systems with nonlinearity via DOBC approach". In: International Journal of Robust and Nonlinear Control 15.3, pp. 109-125 (cit. on p. 24).

Han, Huayun, Ying Yang, Linlin Li, and Steven X Ding (2018). "Observerbased fault detection for uncertain nonlinear systems". In: Journal of the Franklin Institute 355.3, pp. 1278-1295 (cit. on pp. 5, 6, 18).

Han, Jingqing (2009). "From PID to active disturbance rejection control". In: IEEE transactions on Industrial Electronics 56.3, pp. 900-906 (cit. on pp. $4,6,18,50,51)$.

Hansen, Lars Peter and Thomas J Sargent (2008). Robustness. Princeton university press (cit. on pp. 5, 20).

Horowitz, IM (1963). Synthesis oj Feedback Systems (cit. on p. 3).

Huang, Yi and Wenchao Xue (2014). "Active disturbance rejection control: methodology and theoretical analysis". In: ISA transactions 53.4, pp. 963976 (cit. on pp. 4, 10, 50, 51, 53).

Iwasaki, Tetsuya and Robert E Skelton (1994). "All controllers for the general $\mathrm{H} /$ sub infinity / control problem: LMI existence conditions and state space formulas". In: Automatica 30.8, pp. 1307-1317 (cit. on p. 23).

Jain, Shivam and Hote Yogesh (2020). "Generalized active disturbance rejection controller for load frequency control in power systems". In: IEEE Control Systems Letters 4.1, pp. 73-78 (cit. on p. 5). 
Jingqing, Han (1995). "The Extended State Observer of a Class of Uncertain Systems". In: Control and Decision 1, 19-23 (In Chinese) (cit. on p. 24).

Johnson, Carroll D (1975). "On observers for systems with unknown and inaccessible inputs". In: International journal of control 21.5, pp. 825-831 (cit. on p. 4).

- (1986). "Disturbance-accommodating control; an overview". In: 1986 American Control Conference. IEEE, pp. 526-536 (cit. on pp. 4, 24, 28).

Johnson, Cn (1971). "Accomodation of external disturbances in linear regulator and servomechanism problems". In: IEEE Transactions on automatic control 16.6, pp. 635-644 (cit. on pp. 4, 24).

Kadowaki, Satoshi, Kiyoshi Ohishi, Ichiro Miyashita, and Shinobu Yasukawa (2002). "Re-adhesion control of electric motor coach based on disturbance observer and sensor-less vector control". In: Proceedings of the Power Conversion Conference-Osaka 2002 (Cat. No. 02TH8579). Vol. 3. IEEE, pp. 1020-1025 (cit. on p. 5).

Kanderian, Sami S, Stu Weinzimer, Gayane Voskanyan, and Garry M Steil (2009). Identification of intraday metabolic profiles during closed-loop glucose control in individuals with type 1 diabetes (cit. on pp. 10, 20, 42, $43)$.

Kanderian, Sami S, Stuart A Weinzimer, and Garry M Steil (2012). "The identifiable virtual patient model: comparison of simulation and clinical closed-loop study results". In: Journal of diabetes science and technology 6.2 , pp. 371-379 (cit. on pp. 10, 42).

Khalil, Hassan K and Jessy W Grizzle (2002). Nonlinear systems. Vol. 3. Prentice hall Upper Saddle River, NJ (cit. on p. 3).

Khargonekar, Pramod P and Mario A Rotea (1991). "Mixed $\mathcal{H}_{2} / \mathcal{H}_{\infty}$ control: a convex optimization approach". In: IEEE Transactions on Automatic Control 36.7, pp. 824-837 (cit. on pp. 4, 23).

Khlebnikov, Mikhail V (2016). "Control of linear systems subjected to exogenous disturbances: Combined feedback". In: IEEE International Federa- 
tion of Automatic Control (IFAC), pp. 111-116 (cit. on pp. 6, 7, 18, 24, $31,40,53)$.

Kim, Kyung-Soo, Keun-Ho Rew, and Soohyun Kim (2010). "Disturbance observer for estimating higher order disturbances in time series expansion". In: IEEE Transactions on automatic control 55.8, pp. 1905-1911 (cit. on pp. 19, 33, 68).

Krstic, Miroslav (2008). "Lyapunov tools for predictor feedbacks for delay systems: Inverse optimality and robustness to delay mismatch". In: Automatica 44.11, pp. 2930-2935 (cit. on pp. 8, 64).

- (2010). "Compensation of infinite-dimensional actuator and sensor dynamics". In: Control Systems, IEEE 30.1, pp. 22-41 (cit. on pp. 8, 21, 64).

Lamnabhi-Lagarrigue, Francoise, Anuradha Annaswamy, Sebastian Engell, Alf Isaksson, Pramod Khargonekar, Richard M Murray, Henk Nijmeijer, Tariq Samad, Dawn Tilbury, and Paul Van den Hof (2017). "Systems \& Control for the future of humanity, research agenda: Current and future roles, impact and grand challenges". In: Annual Reviews in Control 43, pp. 1-64 (cit. on p. 6).

Léchappé, Vincent, Emmanuel Moulay, Franck Plestan, Alain Glumineau, and Abdelhamid Chriette (2015). "New predictive scheme for the control of LTI systems with input delay and unknown disturbances". In: Automatica 52, pp. 179-184 (cit. on pp. 8, 70, 71).

Lewis, Frank L, Draguna Vrabie, and Vassilis L Syrmos (2012). Optimal control. John Wiley \& Sons (cit. on pp. 28-31).

Li, Shihua, Jun Yang, Wen Hua Chen, and Xisong Chen (2012). "Generalized extended state observer based control for systems with mismatched uncertainties". In: IEEE Transactions on Industrial Electronics 59.12, pp. 4792 4802 (cit. on pp. 6, 7, 10, 18, 31, 39, 40, 45, 53).

Li, Zhao-Yan, Bin Zhou, and Zongli Lin (2014). "On robustness of predictor feedback control of linear systems with input delays". In: Automatica 50.5, pp. 1497-1506 (cit. on pp. 8, 21). 
Liu, Zhijie, Jinkun Liu, and Lijun Wang (2018). "Disturbance observer based attitude control for flexible spacecraft with input magnitude and rate constraints". In: Aerospace Science and Technology 72, pp. 486-492 (cit. on p. 5).

Luenberger, David (1966). "Observers for multivariable systems". In: IEEE Transactions on Automatic Control 11.2, pp. 190-197 (cit. on pp. 33, 68).

Luenberger, David G (1964). "Observing the state of a linear system". In: IEEE transactions on military electronics 8.2 , pp. 74-80 (cit. on pp. 33, 68).

Mahmoud, Chilali and Gahinet Pascal (1996). "H/sub infinity / design with pole placement constraints: an LMI approach". In: IEEE Transactions on Automatic Control 41.3, pp. 358-367 (cit. on p. 23).

Manitius, Andrzej and A Olbrot (1979). "Finite spectrum assignment problem for systems with delays". In: IEEE transactions on Automatic Control 24.4, pp. 541-552 (cit. on pp. 8, 21).

Mayne, David Q (2014). "Model predictive control: Recent developments and future promise". In: Automatica 50.12, pp. 2967-2986 (cit. on pp. 8, 21).

Mazenc, Frederic, Silviu-Iulian Niculescu, and Miroslav Krstic (2012). "LyapunovKrasovskii functionals and application to input delay compensation for linear time-invariant systems". In: Automatica 48.7, pp. 1317-1323 (cit. on pp. 8, 21).

Meditch, JS and GH Hostetter (1973). "Observers for systems with unknown and inaccessible inputs". In: 1973 IEEE Conference on Decision and Control including the 12th Symposium on Adaptive Processes. IEEE, pp. 120124 (cit. on pp. 4, 19, 33, 68).

Michail, Konstantinos (2009). "Optimised configuration of sensing elements for control and fault tolerance applied to an electro-magnetic suspension system". PhD thesis (cit. on pp. 37,38 ).

Natori, Kenji and Kouhei Ohnishi (2008). "A design method of communication disturbance observer for time-delay compensation, taking the dynamic 
property of network disturbance into account". In: IEEE Transactions on Industrial Electronics 55.5, pp. 2152-2168 (cit. on p. 5).

Oh, Yonghwan and Wan Kyun Chung (1999). "Disturbance-observer-based motion control of redundant manipulators using inertially decoupled dynamics". In: IEEE/ASME Transactions on Mechatronics 4.2, pp. 133-146 (cit. on p. 24).

Ohishi, Kiyoshi (1983). "Torque-speed regulation of DC motor based on load torque estimation". In: IEEJ International Power Electronics Conference, IPEC-TOKYO, 1983-3. Vol. 2, pp. 1209-1216 (cit. on pp. 4, 10).

Ohishi, Kiyoshi, Masato Nakao, Kouhei Ohnishi, and Kunio Miyachi (1987). "Microprocessor-controlled DC motor for load-insensitive position servo system". In: IEEE Transactions on Industrial Electronics IE-34.1, pp. 4449 (cit. on p. 24).

Peng, Zhouhua and Jun Wang (2017). "Output-feedback path-following control of autonomous underwater vehicles based on an extended state observer and projection neural networks". In: IEEE Transactions on Systems, Man, and Cybernetics: Systems 48.4, pp. 535-544 (cit. on p. 5).

Petersen, Ian R and Roberto Tempo (2014). "Robust control of uncertain systems: Classical results and recent developments". In: Automatica 50.5, pp. 1315-1335 (cit. on pp. 2, 3, 6, 19, 23).

Polychronopoulos, Aris, Manolis Tsogas, Angelos J Amditis, and Luisa Andreone (2007). "Sensor fusion for predicting vehicles' path for collision avoidance systems". In: IEEE Transactions on Intelligent Transportation Systems 8.3, pp. 549-562 (cit. on pp. 8, 21).

Pu, Zhiqiang, Jinlin Sun, Jianqiang Yi, and Zhiqiang Gao (2020). "On the Principle and Applications of Conditional Disturbance Negation". In: IEEE Transactions on Systems, Man, and Cybernetics: Systems, pp. 1-11 (cit. on p. 7).

Rosenbrock, H and P McMorran (1971). "Good, bad, or optimal?" In: IEEE Transactions on Automatic Control 16.6, pp. 552-554 (cit. on p. 3). 
Ruggiero, Fabio, Vincenzo Lippiello, and Anibal Ollero (2018). "Aerial manipulation: A literature review". In: IEEE Robotics and Automation Letters 3.3 , pp. 1957-1964 (cit. on p. 75 ).

Sadhu, Smita and Tapan Kumar Ghoshal (2010). "Sight line rate estimation in missile seeker using disturbance observer-based technique". In: IEEE Transactions on Control Systems Technology 19.2, pp. 449-454 (cit. on p. 5).

Safonov, Michael G (2012). "Origins of robust control: Early history and future speculations". In: Annual Reviews in Control 36.2, pp. 173-181 (cit. on pp. 2,3).

Safonov, Michael George (1980). Stability and robustness of multivariable feedback systems. MIT press (cit. on p. 3).

Sala-Mira, Ivan, Jose-Luis Diez, Beatriz Ricarte, and Jorge Bondia (2019). "Sliding-mode disturbance observers for an artificial pancreas without meal announcement". In: Journal of Process Control 78, pp. 68-77 (cit. on p. 5).

Sandberg, I Wo (1964). "A frequency-domain condition for the stability of feedback systems containing a single time-varying nonlinear element". In: Bell System Technical Journal 43.4, pp. 1601-1608 (cit. on p. 3).

Santos, Tito LM and Taniel S Franklin (2018). "Enhanced Finite Spectrum Assignment with disturbance compensation for LTI systems with input delay". In: Journal of the Franklin Institute 355.8, pp. 3541-3566 (cit. on pp. 8, 21).

Sanz, Ricardo, Pedro Garcia, and Pedro Albertos (2016a). "Enhanced disturbance rejection for a predictor-based control of LTI systems with input delay". In: Automatica 72, pp. 205-208 (cit. on pp. 70-72).

Sanz, Ricardo, Pedro García, José-Luis Díez, and Jorge Bondia (2020). "Artificial Pancreas System With Unannounced Meals Based on a Disturbance Observer and Feedforward Compensation". In: IEEE Transactions on Control Systems Technology (cit. on pp. 5, 10). 
Sanz, Ricardo, Pedro Garcia, Qing-Chang Zhong, and Pedro Albertos (2016b). "Predictor-based control of a class of time-delay systems and its application to quadrotors". In: IEEE Transactions on Industrial Electronics 64.1, pp. 459-469 (cit. on pp. 6, 8, 18, 21, 70).

Sariyildiz, Emre, Gong Chen, and Haoyong Yu (2015). "An acceleration-based robust motion controller design for a novel series elastic actuator". In: IEEE Transactions on Industrial Electronics 63.3, pp. 1900-1910 (cit. on p. 5).

Sariyildiz, Emre, Roberto Oboe, and Kouhei Ohnishi (2020). "Disturbance observer-based robust control and its applications: 35th anniversary overview". In: IEEE Transactions on Industrial Electronics 67.3, pp. 2042-2053 (cit. on pp. $2-4,6,7,9,10,24)$.

Scherer, Carsten and Siep Weiland (2000). "Linear matrix inequalities in control". In: Lecture Notes, Dutch Institute for Systems and Control, Delft, The Netherlands 3.2 (cit. on pp. 3, 35, 70).

Schweppe, Fred (1968). "Recursive state estimation: Unknown but bounded errors and system inputs". In: IEEE Transactions on Automatic Control 13.1 , pp. 22-28 (cit. on pp. 4, 33, 68).

She, Jin-Hua, Mingxing Fang, Yasuhiro Ohyama, Hiroshi Hashimoto, and Min $\mathrm{Wu}$ (2008). "Improving disturbance-rejection performance based on an equivalent-input-disturbance approach". In: IEEE Transactions on Industrial Electronics 55.1, pp. 380-389 (cit. on pp. 6, 7, 18, 24, 53).

Shim, Hyungbo and Nam H Jo (2009). "An almost necessary and sufficient condition for robust stability of closed-loop systems with disturbance observer". In: Automatica 45.1, pp. 296-299 (cit. on pp. 6, 18, 24).

Stocker, David, Sami Kanderian, Gabriel Cortina, Tamir Nitzan, Jon Plummer, Garry Steil, and John Mastrototaro (2006). Virtual patient software system for educating and treating individuals with diabetes (cit. on pp. 10, 42).

Strategic research agenda for robotics in Europe (2014-2020). Tech. rep. (cit. on p. 75). 
Su, Jinya and Wen-Hua Chen (2018). "Further results on "Reduced order disturbance observer for discrete-time linear systems"". In: Automatica 93, pp. 550-553 (cit. on pp. 19, 33, 68).

Tadmor, Gilead (1990). "Worst-case design in the time domain: The maximum principle and the standard $\mathrm{H} /$ sub infinity / problem". In: Mathematics of Control, Signals and Systems 3.4, pp. 301-324 (cit. on pp. 19, 23).

Tecnodiabetes ai2, UPV. http://tecnodiabetes . ai2 . upv . es/. Accessed: 2020 (cit. on pp. 10, 75).

Tomizuka, Masayoshi (1974). "The optimal finite preview problem and its application to man-machine systems." PhD thesis. Massachusetts Institute of Technology (cit. on p. 23).

- (1975). "Optimal continuous finite preview problem". In: IEEE transactions on automatic control 20.3, pp. 362-365 (cit. on p. 23).

Ugurlu, Barkan, Masayoshi Nishimura, Kazuyuki Hyodo, Michihiro Kawanishi, and Tatsuo Narikiyo (2014). "Proof of concept for robot-aided upper limb rehabilitation using disturbance observers". In: IEEE Transactions on Human-Machine Systems 45.1, pp. 110-118 (cit. on p. 5).

Umeno, Takaji and Yoichi Hori (1991). "Robust speed control of DC servomotors using modern two degrees-of-freedom controller design". In: IEEE Transactions on industrial electronics 38.5, pp. 363-368 (cit. on p. 5).

Wang, H and S Daley (1996). "Actuator fault diagnosis: an adaptive observerbased technique". In: IEEE transactions on Automatic Control 41.7, pp. 10731078 (cit. on pp. 5, 6, 18).

Wu, Ze-Hao, Hua-Cheng Zhou, Bao-Zhu Guo, and Feiqi Deng (2020). "Review and new theoretical perspectives on active disturbance rejection control for uncertain finite-dimensional and infinite-dimensional systems". In: Nonlinear Dynamics, pp. 1-25 (cit. on pp. 4, 10, 50).

Xie, Liang-Liang and Lei Guo (2000). "How much uncertainty can be dealt with by feedback?" In: IEEE Transactions on Automatic Control 45.12, pp. 2203-2217 (cit. on p. 50). 
Xiong, Yi and Mehrdad Saif (2003). "Unknown disturbance inputs estimation based on a state functional observer design". In: Automatica 39.8, pp. 1389-1398 (cit. on pp. 19, 33, 68).

Yang, Jun, Argyrios Zolotas, Wen-Hua Chen, Konstantinos Michail, and Shihua Li (2011). "Robust control of nonlinear MAGLEV suspension system with mismatched uncertainties via DOBC approach". In: ISA transactions 50.3, pp. 389-396 (cit. on pp. 5, 7, 10, 31, 37-39, 41, 45).

Yokoyama, Tomoki and Atsuo Kawamura (1994). "Disturbance observer based fully digital controlled PWM inverter for CVCF operation". In: IEEE Transactions on Power Electronics 9.5, pp. 473-480 (cit. on p. 5).

Zames, George (1966). "On the input-output stability of time-varying nonlinear feedback systems part one: Conditions derived using concepts of loop gain, conicity, and positivity". In: IEEE transactions on automatic control 11.2, pp. 228-238 (cit. on p. 3).

Zhong, Qing-Chang and David Rees (2004). "Control of uncertain LTI systems based on an uncertainty and disturbance estimator". In: ASME Journal of Dynamic Systems, Measurement, and Control 126.4, pp. 905-910 (cit. on p. 24).

Zhou, Kemin, John Comstock Doyle, Keith Glover, et al. (1996). Robust and optimal control. Vol. 40. Prentice hall New Jersey (cit. on p. 23).

Zhou, Ping, Wei Dai, and Tian-You Chai (2014). "Multivariable disturbance observer based advanced feedback control design and its application to a grinding circuit". In: IEEE Transactions on Control Systems Technology 22.4 , pp. $1474-1485$ (cit. on p. 24). 


\section{Annex 1. Proof of Theorem 4.2.1}

By Lemma 4.2.1. Let us consider the Lyapunov function $V_{i}(\xi)=\xi^{T} P_{i} \xi, P_{i}>0$, and let us set $g_{1}(x, t)=\omega(x, \tilde{\omega}(t)), g_{2}(x, t)=\frac{\partial \omega}{\partial \tilde{\omega}}(x, \tilde{\omega}(t)) \dot{\tilde{\omega}}(t)$ with $\alpha_{1}=\beta_{f}$ and $\alpha_{2}=\beta_{d \tilde{\omega}} \beta_{\dot{\tilde{\omega}}}$. If there exist $\gamma_{1_{i}}>0, \gamma_{2_{i}}>0, \delta_{i}>0$ such that

$$
\dot{V}_{i}+\delta_{i} V_{i}-\gamma_{1_{i}}\|\omega(x, \tilde{\omega}(t))\|-\gamma_{2_{i}}\left\|\frac{\partial \omega}{\partial \tilde{\omega}}(x, \tilde{\omega}(t)) \dot{\tilde{\omega}}(t)\right\|^{2} \leq 0,
$$

for all $t \geq t_{0}$, then, the ellipsoid

$$
\mathcal{E}_{i} \triangleq\left\{\xi \in \mathbb{R}^{2 n+q} \mid \xi^{T} P_{i} \xi \leq \frac{\gamma_{1_{i}} \beta_{f}^{2}+\gamma_{2_{i}}\left(\beta_{d \tilde{\omega}} \beta_{\dot{\tilde{\omega}}}\right)^{2}}{\delta_{i}}\right\}
$$

is positively invariant and exponentially attractive.

Hence, the proof is reduced to show how (4.8)-(4.9) imply (5.20). The derivative of $V_{i}$, with $\dot{\xi}$ substituted by (4.6) is

$$
\begin{aligned}
\dot{V}_{i}= & \xi^{T}\left(P_{i} \Phi_{c}+\Phi_{c}^{T} P_{i}\right) \xi+2 \xi^{T} P_{i} \Gamma_{1} f+2 \xi^{T} P_{i} \Gamma_{2} d f_{x} \\
& +2 \xi^{T} P_{i} \Gamma_{2} d f_{t},
\end{aligned}
$$

where, for simplicity, $\omega \triangleq \omega(x, \tilde{\omega}(t)), d f_{x} \triangleq \frac{\partial \omega}{\partial x}(x, \tilde{\omega}(t)) \dot{x}$, and $d f_{t} \triangleq \frac{\partial \omega}{\partial \tilde{\omega}}(x, \tilde{\omega}(t)) \dot{\tilde{\omega}}(t)$.

Substituting (5.22) into (5.20) leads to

$$
\begin{aligned}
\xi^{T}\left(P_{i} \Phi_{c}\right. & \left.+\Phi_{c}^{T} P_{i}\right) \xi+2 \xi^{T} P_{i} \Gamma_{1} f+2 \xi^{T} P_{i} \Gamma_{2} d f_{x}+ \\
& +2 \xi^{T} P_{i} \Gamma_{2} d f_{t}+\delta_{i} \xi^{T} P_{i} \xi-\gamma_{1_{i}} \omega^{T} f-\gamma_{2_{i}} d f_{t}^{T} d f_{t} \leq 0,
\end{aligned}
$$


being expressed as $\phi^{T}\left(\Psi_{i s s, 0}^{i}\right) \phi \leq 0, \phi \triangleq\left[\xi^{T}, \omega, d f_{x}, d f_{t}\right]^{T}$,

$$
\Psi_{i s s, 0}^{i} \triangleq\left[\begin{array}{cccc}
P_{i} \Phi_{c}+\Phi_{c}^{T} P_{i}+\delta_{i} P_{i} & P_{i} \Gamma_{1} & P_{i} \Gamma_{2} & P_{i} \Gamma_{2} \\
(*) & -\gamma_{1_{i}} & 0 & 0 \\
(*) & (*) & 0 & 0 \\
(*) & (*) & (*) & -\gamma_{2_{i}}
\end{array}\right]
$$

So, if $\Psi_{i s s, 0}^{i} \leq 0$, then (5.20) is satisfied. The next step follows by the application of the $S$-procedure in the term $d f_{x}$. It is known that

$$
\begin{aligned}
d f_{x} & =\frac{\partial \omega}{\partial x}(x, \tilde{\omega}(t))\left[A x+B_{2} u+B_{1} f\right] \\
& =\frac{\partial \omega}{\partial x}(x, \tilde{\omega}(t))\left[A x+B_{2}\left(K_{x}\left(x-e_{o, x}\right)+K_{\omega, 0}\left(\omega-e_{o, \omega}\right)\right)+B_{1} f\right] \\
& =\frac{\partial \omega}{\partial x}(x, \tilde{\omega}(t))\left[\Delta_{\xi} \xi+\Delta_{f} f\right],
\end{aligned}
$$

with $\Delta_{\xi} \triangleq\left[\left(A+B_{2} K_{x}\right), B_{2} E_{\eta}\right], \Delta_{\omega} \triangleq\left(B_{2} K_{\omega, 0}+B_{1}\right)$.

Hence, by Assumption 4.1 and for all $x \in \mathcal{B}_{r}$, the following upper bound can be established:

$$
d f_{x}^{T} d f_{x} \leq \beta_{d x}^{2}\left[\xi^{T} \Delta_{\xi}^{T} \Delta_{\xi} \xi+2 \xi^{T} \Delta_{\xi}^{T} \Delta_{f} f+\omega^{T} \Delta_{f}^{T} \Delta_{f} f\right],
$$

which can be written as $\phi^{T}\left(\Psi_{i s s, 1}^{i}\right) \phi \leq 0$, with

$$
\Psi_{i s s, 1}^{i} \triangleq\left[\begin{array}{cccc}
-\beta_{d x}^{2} \Delta_{\xi}^{T} \Delta_{\xi} & -\beta_{d x}^{2} \Delta_{\xi}^{T} \Delta_{f} & 0 & 0 \\
(*) & -\beta_{d x}^{2} \Delta_{f}^{T} \Delta_{f} & 0 & 0 \\
(*) & (*) & 1 & 0 \\
(*) & (*) & (*) & 0
\end{array}\right]
$$

The knowledge of $\Psi_{i s s, 1}^{i} \leq 0$ implies that $\Psi_{i s s, 0}^{i} \leq 0$ if there exist $\tau_{i}>0$ such that $\phi^{T} \Psi_{i s s, 0}^{i} \phi \leq \tau_{i} \phi^{T} \Psi_{i s s, 1}^{i} \phi \leq 0$. This holds if $\phi^{T}\left(\Psi_{i s s, 0}^{i}-\Psi_{i s s, 1}^{i}\right) \phi \leq 0$, leading to (4.8).

So, if (4.8) holds, then the ellipsoid (5.21) is attractive and positively invariant. Condition (4.9) follows from a short analysis of the sets that are being considered in this problem. Lemma 4.2.1 considers that $g_{1}(x, t)=\omega(x, \tilde{\omega}(t))$ and $g_{2}(x, t)=\frac{\partial \omega}{\partial \tilde{\omega}}(x, \tilde{\omega}(t)) \dot{\tilde{\omega}}(t)$ are bounded. However, by Assumption 4.1, this bounds can be only established in $\mathcal{B}_{r}$. So it must be required that $x(t)$ lies inside $\mathcal{B}_{r}$ for all $t \geq t_{0}$. 
As mentioned in Section 4.1, the ellipsoid (5.21) has a projection onto $\mathbb{R}^{n}$ given by

$$
\mathcal{E}_{i}^{\perp} \triangleq\left\{x \in \mathbb{R}^{n} \mid x^{T} P_{i}^{\perp} x \leq \frac{\gamma_{1_{i}} \beta_{f}^{2}+\gamma_{2_{i}}\left(\beta_{d \tilde{\omega}} \beta_{\dot{\omega}}\right)^{2}}{\delta_{i}}\right\} .
$$

Therefore, it must be imposed that $\mathcal{E}_{i}^{\perp} \subseteq \mathcal{B}_{r}$, which is satisfied if (4.9) holds. Finally, the theorem follows by defining two independent solutions, given by $i \triangleq\{0, \infty\}$, such that both satisfy (4.8)-(4.9). In this case, two attractive ellipsoids, i.e $\mathcal{E}_{0}, \mathcal{E}_{\infty}$; are obtained. If the initial state is restricted to be inside $\mathcal{E}_{0}$, then $x(t) \in \mathcal{B}_{r}$ for all $t \geq t_{0}$, and $x(t)$ approaches to $\mathcal{E}_{\infty}$ with a exponential rate $\delta_{\infty} / 2$. 



\section{Annex 2. MatLab ${ }^{\circledR}$ implementations}

Computation of the DOBCs - matrices in Thm. 3.3.1

The following code implements a MatLab function whose inputs are the model matrices $\left(A, B_{1}, B_{2}, C_{1}\right)$, in (2.8), plus the controller tuning parameters $(R, \gamma, r)$ -defined in (2.9) and (3.12) - and it gives as an output the matrices $K_{x}$ and $K_{\omega}=K_{v} \Phi$; which define de DOBC (3.10).

function $[\mathrm{Kx}, \mathrm{Kw}]=\operatorname{getKxKw}(\mathrm{A}, \mathrm{B} 1, \mathrm{~B} 2, \mathrm{C} 1, \mathrm{R}, \mathrm{gam}, \mathrm{r})$

$\%$ Compute matrices

$\mathrm{S} \_\mathrm{T}=\operatorname{care}\left(\mathrm{A}, \mathrm{B} 2, \mathrm{C} 1{ }^{\prime} * \mathrm{C} 1, \operatorname{gam} * \mathrm{R}\right) ; \%$ Solve $\operatorname{ARE}(3.2)$.

$\overline{\mathrm{Kx}}=-\operatorname{inv}(\operatorname{gam} * \mathrm{R}) * \mathrm{~B} 2^{\prime} * \mathrm{~S}_{-} \mathrm{T}$;

$\mathrm{Kv}=-\operatorname{inv}(\operatorname{gam} * \mathrm{R}) * \mathrm{~B} 2^{\prime}$;

$\mathrm{Acl}=\mathrm{A}+\mathrm{B} 2 * \mathrm{Kx}$

Phi $=[]$;

for $\mathrm{i}=1: 1: \mathrm{r}+1$

Phi $=\operatorname{horzcat}\left(\operatorname{Phi},\left(-\mathrm{Acl}^{\prime}\right)^{\wedge}{ }^{\wedge}(-\mathrm{i}) * \mathrm{~S} \_\mathrm{T} * \mathrm{~B} 1\right)$;

end

$\mathrm{Kw}=\mathrm{Kv} * \mathrm{Phi}$;

end 


\section{Computation of the DOBPs - matrices in Thm. 5.2.1}

The next function computes the matrix $\Gamma_{\omega, i}\left(\Delta_{t}\right)$ by receiving as inputs: i) the nominal-model matrices $\left(A, B_{1}\right)$; ii) the prediction-horizon Delta_t $=\Delta_{t}$; and iii) the integer $i$, which refers to the $i$ th-disturbance derivative to which this matrix multiplies in Eq. (5.11).

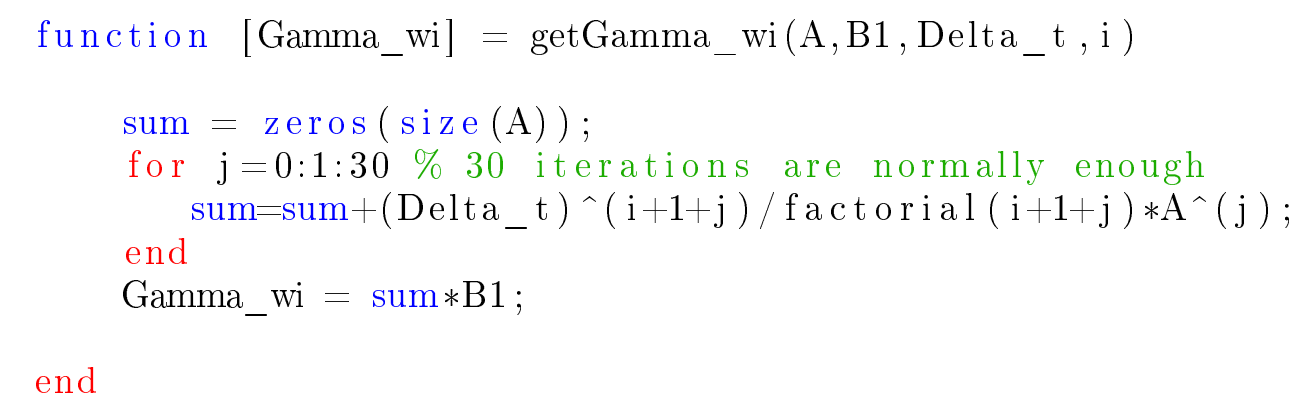

In order to compute $\boldsymbol{\Gamma}_{\boldsymbol{\omega}}\left(\Delta_{t}\right) \triangleq\left[\Gamma_{\omega, 0}\left(\Delta_{t}\right), \Gamma_{\omega, 1}\left(\Delta_{t}\right), \ldots, \Gamma_{\omega, r}\left(\Delta_{t}\right)\right]$, this function can be $(r+1)$-times recursively called as follows:

1 Gamma_w $=[]$;

2 for $i=0: 1: r$

4 end

Gamma_w $=$ horzcat $($ Gamma_w, getGamma_wi $(\mathrm{A}, \mathrm{Bw}, \mathrm{h}, \mathrm{i}))$;

Similarly, the matrix $\Gamma_{u}\left(\tilde{t}_{k}, \tilde{t}_{k+1}\right)$ can be computed through the next function, which receives as inputs: i) the nominal-model matrices $\left(A, B_{2}\right)$; ii) the time values tilde_tk $=\tilde{t}_{k} \triangleq t_{k}+h$, tilde_tk1 $=\tilde{t}_{k+1} \triangleq t_{k+1}+h$; and iii) tPlusDelta_t $=t+\Delta_{t}$.

1 function [GammU_tk_tk1] = getGamma_u(A,B2,tilde_tk, tilde_tk1, tPlusse-jelta_t)

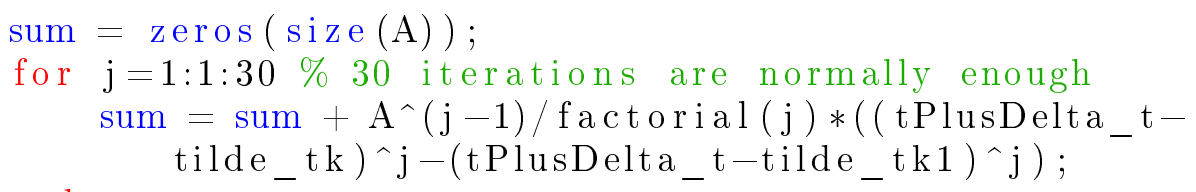


In this way, in order to compute

$$
\boldsymbol{\Gamma}_{\boldsymbol{u}}\left(\tilde{t}_{0}, \ldots, \tilde{t}_{N+1}\right) \triangleq\left[\Gamma_{u}\left(\tilde{t}_{0}, \tilde{t}_{1}\right), \Gamma_{u}\left(\tilde{t}_{1}, \tilde{t}_{2}\right), \ldots, \Gamma_{u}\left(\tilde{t}_{N}, \tilde{t}_{N+1}\right)\right]
$$

one just needs to call repeatedly to the above function.

Note that, in order to compute $\boldsymbol{\Gamma}_{\boldsymbol{u}}$, it is needed to know beforehand all the timeinstants, $t_{k}$, in which the control action $u_{k}$ has been -or will be- introduced to the system — as defined in Def. 5.1. If the system has input-delays, this requires to store in memory some past time instants and their associated control actions. If we are computing predictions so that $\Delta_{t}>h$, then the future timeinstants in which the control action will change should be also established.

\section{High-order ESO design — optimization of Lem. 3.4.1}

The following code implements a MatLab function that optimizes the LMI problem in Lem. 3.4.1.

Its inputs are: i) the model matrices $\left(A, B_{1}, B_{2}, C_{2}\right)$ in $(2.8)$; ii) the high-order ESO tuning parameters $(r, K, M, N)$ - which are defined in Lem. 3.4.1; and iii) the optimizing parameter $\delta$. The function employs Yalmip +Sedumi in order to solve the LMI problem (3.20a)-(3.20c); maximizing $\alpha$ for a fixed value of $\delta$ - as suggested in Rmk 3.1. The function could be repeatedly called for increasing values of $\delta$ until the maximum $\delta \alpha$ is found.

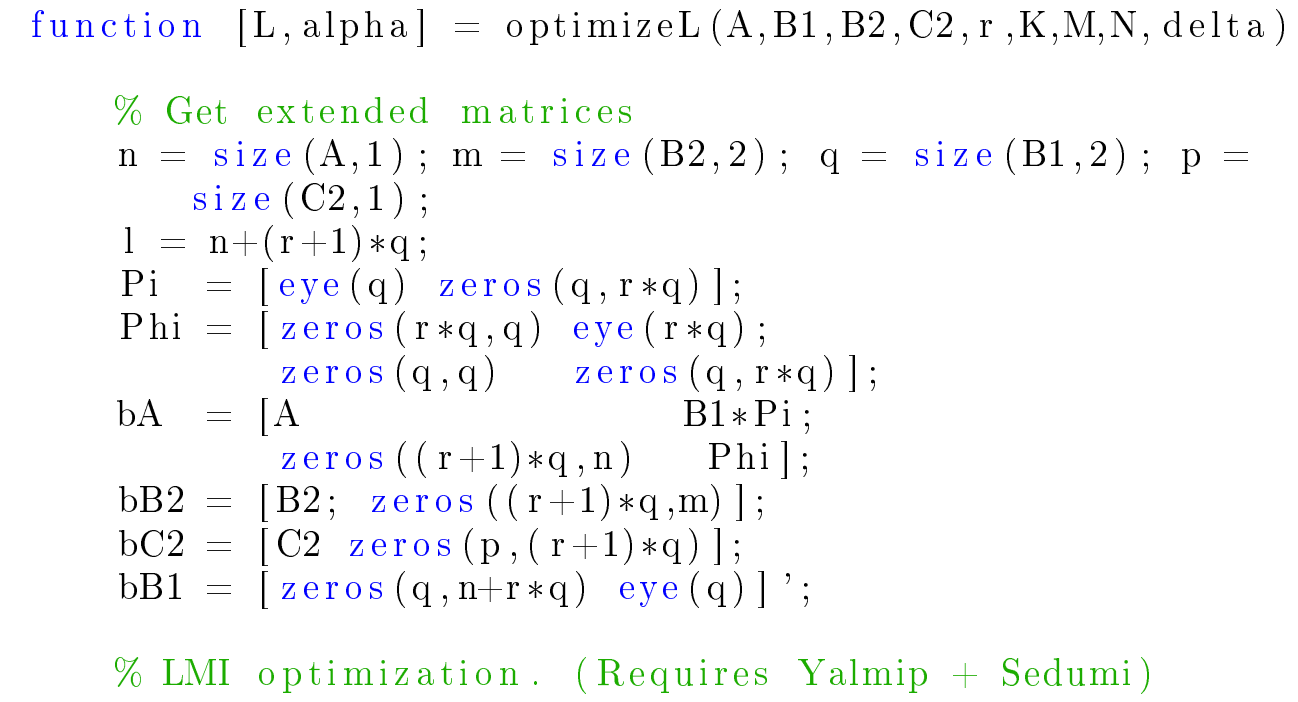


In this sense, once the nominal model matrices $\left(A, B_{1}, B_{2}, C_{2}\right)$ have been defined, this function can be used to get the gain $L$ of the high-order ESO (3.17); which could then be constructed with the matrices $\mathrm{bA}=\bar{A}, \mathrm{bB} 2=\bar{B}_{2}, \mathrm{bC} 2=\bar{C}_{2}$. The works by Chilali et al. 1996; Chilali et al. 1999 include some examples of matrices $M, N$, which enforce that the eigenvalues of $(\bar{A}-L \bar{C})$ belong to a specific region of the complex plane.

In the case of using this function to construct a high-order ESO-based controller as in Sec. 3.4, it should be called with $K=\left[K_{x}, K_{v} \Phi\right]$. In the case of using it to construct a high-order ESO-based predictor as in Sec. 5.3, it should be called with $K=\Psi\left(\Delta_{t}\right) \triangleq\left[\Phi\left(\Delta_{t}\right), \boldsymbol{\Gamma}_{\boldsymbol{\omega}}\left(\Delta_{t}\right)\right]$. If there is no specific application, one could just call it with $K=I_{l}$

Finally, the above code can be easily reconfigured in order to implement the LMI optimizations of the stability conditions that were reported in Sec. 4.3. 